\title{
Co(II) PCP Pincer Complexes as Catalysts for the Alkylation of Aromatic Amines with Primary Alcohols
}

Matthias Mastalir, ${ }^{\dagger}$ Gerald Tomsu, $^{\dagger}$ Ernst Pittenauer, $^{\ddagger}$ Günter Allmaier, ${ }^{\ddagger}$ Karl Kirchner ${ }^{*} \dagger$

${ }^{\dagger}$ Institute of Applied Synthetic Chemistry and ${ }^{\ddagger}$ Institute of Chemical Technologies and Analytics, Vienna University of Technology, Getreidemarkt 9, A-1060 Vienna, AUSTRIA

1. General Information

2. Synthesis and Characterization of Catalysts

3. General Procedure for the Alkylation of Amines

4. Characterization of Organic Products.

5. References

6. ${ }^{1} \mathrm{H}$ and ${ }^{13} \mathrm{C}\left\{{ }^{1} \mathrm{H}\right\}$ NMR Spectra of Organic Products 


\section{General Information}

All manipulations were performed under an inert atmosphere of argon by using Schlenk techniques or in a MBraun inert-gas glovebox. The solvents were purified according to standard procedures. ${ }^{1}$ The deuterated solvents were purchased from Aldrich and dried over $3 \AA$ molecular sieves. Complexes $\left[\mathrm{Co}\left(\mathrm{PCP}^{\mathrm{Me}}{ }_{-}-\mathrm{Pr}\right) \mathrm{Cl}\right](\mathbf{1})$ and $\left[\mathrm{Co}\left(\mathrm{PCP}^{\mathrm{Me}}{ }_{-} \mathrm{Pr}\right) \mathrm{Cl}_{2}\right](2)$ were prepared according to the literature. $^{2}$ All substrates are known compounds and were used as obtained from commercial resources and purified bevore usage. ${ }^{1} \mathrm{H}$ and ${ }^{13} \mathrm{C}\left\{{ }^{1} \mathrm{H}\right\},{ }^{19} \mathrm{~F}\left\{{ }^{1} \mathrm{H}\right\}$ and ${ }^{31} \mathrm{P}\left\{{ }^{1} \mathrm{H}\right\}$ NMR spectra were recorded on Bruker AVANCE-250 and AVANCE-400 spectrometers. ${ }^{1} \mathrm{H}$ and ${ }^{13} \mathrm{C}\left\{{ }^{1} \mathrm{H}\right\}$ NMR spectra were referenced internally to residual protio-solvent, and solvent resonances, respectively, and are reported relative to tetramethylsilane $(\sigma=0 \mathrm{ppm}) .{ }^{31} \mathrm{P}\left\{{ }^{1} \mathrm{H}\right\}$ NMR spectra were referenced externally to $\mathrm{H}_{3} \mathrm{PO}_{4}$ $(85 \%)(\sigma=0 \mathrm{ppm})$. As reaction vessel $8 \mathrm{~mL}$ microwave vials from Biotage or VWR with aluminium septum cap were used for the $140^{\circ} \mathrm{C}$ reactions and $8 \mathrm{~mL}$ screw cap glass vials for the basic reactions.

Room-temperature solution magnetic moments were determined by ${ }^{1} \mathrm{H}$ NMR spectroscopy using the method of Evans (THF- $\left.\mathrm{d}_{8}\right){ }^{3}$

ESI MS measurements were performed on an Esquire $3000^{\text {plus }} 3 \mathrm{D}$-quadrupole ion trap mass spectrometer (Bruker Daltonics, Bremen, Germany) in positive-ion mode by means of electrospray ionization (ESI). Mass calibration was done with a commercial mixture of perfluorinated trialkyltriazines (ES Tuning Mix, Agilent Technologies, Santa Clara, CA, USA). All analytes were dissolved in $\mathrm{ACN} / \mathrm{H}_{2} \mathrm{O} / \mathrm{HCOOH}$ "hypergrade for LC-MS Lichrosolv" quality (Merck, Darmstadt, Germany) to form a concentration of roughly $1 \mathrm{mg} / \mathrm{mL}$ in order to suppress dehydrogenations of several analytes. Direct infusion experiments were carried out using a Cole Parmer model 74900 syringe pump (Cole Parmer Instruments, Vernon Hills, IL, USA) at a flow rate of $2 \mu \mathrm{L} / \mathrm{min}$. Full scan and MS/MS (low energy CID)scans were measured in the range $\mathrm{m} / \mathrm{z} 100-1100$ with the target mass set to $\mathrm{m} / \mathrm{z} 1000$. Further experimental conditions include: drying gas temperature: $200^{\circ} \mathrm{C}$; capillary voltage: $-4 \mathrm{kV}$; skimmer voltage: $40 \mathrm{~V}$; octapole and lens voltages: according to the target mass set. Mass spectra were averaged during data acquisition time of 1 to $2 \mathrm{~min}$ and one analytical scan consisted of five successive micro scans resulting in 50 and 100 analytical scans, respectively, for the final full scan mass spectrum. High resolution mass spectra were recorded on a Bruker ESI-Qq aoTOF MS spectrometer.

\section{Synthesis and Characterization of Catalysts}

[Co(PCP ${ }^{\mathrm{Me}}{ }_{-i \mathrm{Pr}}$ (Me)] (3). A solution of [Co(PCP $\left.\left.{ }^{\mathrm{Me}}{ }_{-i \mathrm{Pr}}\right) \mathrm{Cl}\right](\mathbf{1})(100 \mathrm{mg}, 0.22 \mathrm{mmol})$ in toluene $(5 \mathrm{~mL})$ was treated with MeLi (1.6 M in diethyl ether, $0.135 \mathrm{~mL}, 0.22 \mathrm{mmol})$ and stirred for $16 \mathrm{~h}$ at room temperature. After that, the volume of the brown solution was reduced to about $2 \mathrm{~mL}, n$-pentane (6 $\mathrm{mL}$ ) was added, and a brown precipitate was formed which was filtered off. The solvent was then removed under reduced pressure and $\mathbf{3}$ was obtained as a brown powder. Yield: $50 \mathrm{mg}$ (52\%). Anal. Calcd. for $\mathrm{C}_{21} \mathrm{H}_{40} \mathrm{CoN}_{2} \mathrm{P}_{2}$ (441.45): C, 57.14; $\mathrm{H}, 9.13 ; \mathrm{N}, 6.35$. Found: $\mathrm{C}, 57.26 ; \mathrm{H}, 9.34 ; \mathrm{N}, 6.40 . \mu_{\text {eff }}=$ $2.34 \mu_{\mathrm{B}}$. $\left(\mathrm{d}_{8}-\mathrm{THF}\right)$. This value is higher than the one expected for the spin-only approximation and is explained by a spin orbit coupling contribution, being consistent with a low-spin square planar complex. ${ }^{4}$ 
[Co(PCP ${ }^{\mathrm{Me}}-$ iPr) $\left(\mathrm{CH}_{2} \mathrm{SiMe}_{3}\right)$ ] (4). To a suspension of [Co(PCP $\left.{ }^{\mathrm{Me}}-i \mathrm{Pr}\right) \mathrm{Cl}$ (1) (100 mg, 0.22 mmol) in $n$-pentan $(5 \mathrm{~mL}) \mathrm{LiCH}_{2} \mathrm{SiMe}_{3}(1.0 \mathrm{M}$ in $n$-pentane, $0.207 \mathrm{~mL}, 0.21 \mathrm{mmol})$ was added. The mixture was stirred for $16 \mathrm{~h}$ at room temperature. The precipitate was filtered off and the solvent was removed under reduced pressure and a red powder was obtained. Yield: $57 \mathrm{mg}(57 \%)$. Anal. Calcd. for $\mathrm{C}_{24} \mathrm{H}_{48} \mathrm{CoN}_{2} \mathrm{P}_{2} \mathrm{Si}$ (513.63): C, 56.12; $\mathrm{H}, 9.42 ; \mathrm{N}, 5.45$. Found: $\mathrm{C}, 56.02 ; \mathrm{H}, 9.20 ; \mathrm{N}, 5.5 . \mu_{\text {eff }}=2.38$ $\mu_{\mathrm{B}}$. $\left(\mathrm{d}_{8}-\mathrm{THF}\right)$. This value is higher than the one expected for the spin-only approximation and is explained by a spin orbit coupling contribution, being consistent with a low-spin square planar complex.

\section{General Procedure for the Alkylation of Amines}

Methodology 1. Alcohol (1.0 mmol), aniline $(1.4 \mathrm{mmol})$ and $t$-BuOK $(1.3 \mathrm{mmol})$ were mixed in toluene $(4 \mathrm{~mL})$ and the catalyst $(0.02 \mathrm{mmol}, 2 \mathrm{~mol} \%)$ was added under inert conditions. After $16 \mathrm{~h}$ at $80{ }^{\circ} \mathrm{C}$ the mixture was quenched with water (ca. $2 \mathrm{~mL}$ ), the organic layer was dried with $\mathrm{MgSO}_{4}$ and purified via silica column chromatography (eluted with toluene and $\left.\mathrm{Et}_{2} \mathrm{O}\right)$.

Methodology 2. Alcohol $(1.0 \mathrm{mmol})$, aniline $(1.4 \mathrm{mmol})$ and $3 \AA \mathrm{MS}(0.2 \mathrm{~g})$ were mixed in toluene $(4 \mathrm{~mL})$ and the catalyst $(0.02 \mathrm{mmol}, 2 \mathrm{~mol} \%)$ was added under inert conditions. After $16 \mathrm{~h}$ at $130^{\circ} \mathrm{C}$ the mixture was directly purified via silica column chromatography (eluted with toluene and $\mathrm{Et}_{2} \mathrm{O}$ ).

\section{Characterization of Organic Products.}

\section{N-Benzylbenzeneamine (5)}

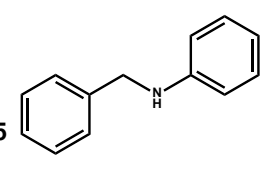

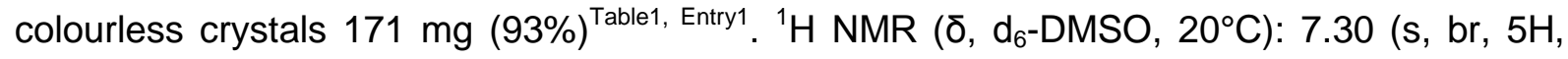
$\mathrm{PhH}$ ), 6.99 (s, br, 1H, PhH), 6.53 (s, br, 3H, PhH), 6.16 (s, br, 1H, NH), 4.20 (s, br, 2H, $\mathrm{CH}_{2}$ ). ${ }^{13} \mathrm{C}\left\{{ }^{1} \mathrm{H}\right\}$ NMR $\left(\delta, \mathrm{d}_{6}\right.$-DMSO, $\left.20^{\circ} \mathrm{C}\right): 150.4,142.1,130.6,130.0,128.9,128.3,117.5,114.0$, 48.2. ESI-MS: $[\mathrm{M}+\mathrm{H}]^{+}$, found 184.11. $\left[\mathrm{C}_{13} \mathrm{H}_{14} \mathrm{~N}\right]^{+}$requires 184.11, MS-MS: 106.27, 91.39.

\section{$\mathrm{N}$-Ethylbenzeneamine (6) ${ }^{6}$}

yellow oil $112 \mathrm{mg}(92 \%) .{ }^{1} \mathrm{H}$ NMR (ס, $\left.\mathrm{d}_{6}-\mathrm{DMSO}, 20^{\circ} \mathrm{C}\right):$ 7.11-7.05 (m, 2H, PhH), 6.59-6.51 $\left.(\mathrm{m}, 3 \mathrm{H}, \mathrm{PhH}), 3.35-3.25\left(\mathrm{~m}, 2 \mathrm{H}, \mathrm{CH}_{2}\right), 1.16\left(\mathrm{t}, \mathrm{J}=1.2 \mathrm{~Hz}, 3 \mathrm{H}, \mathrm{CH}_{3}\right) .{ }^{13} \mathrm{C}^{1} \mathrm{H}\right\} \operatorname{NMR}\left(\delta, \mathrm{d}_{6^{-}}\right.$ DMSO, $20^{\circ} \mathrm{C}$ ): 150.7, 130.5, 117.2, 113.7, 39.1, 16.1. ESI-MS: $[\mathrm{M}+\mathrm{H}]^{+}$, found 122.25 . $\left[\mathrm{C}_{8} \mathrm{H}_{12} \mathrm{~N}\right]^{+}$requires 122.10, MS-MS: 94.06. 
$\mathrm{N}, \mathrm{N}$-Diethylbenzeneamine (7) ${ }^{7}$

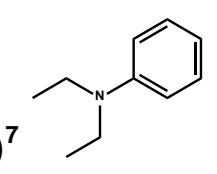

orange oil $14.7 \mathrm{mg}(10 \%) .{ }^{1} \mathrm{H}$ NMR $\left(\delta, \mathrm{d}_{6}-\mathrm{DMSO}, 20^{\circ} \mathrm{C}\right): 7.19-7.10(\mathrm{~m}, 2 \mathrm{H}, \mathrm{PhH}), 6.67-6.52$ $(\mathrm{m}, 3 \mathrm{H}, \mathrm{PhH}), 5.38(\mathrm{~s}, \mathrm{br}, 1 \mathrm{H}, \mathrm{NH}), 3.07-2.96\left(\mathrm{~m}, 2 \mathrm{H}, \mathrm{CH}_{2}\right), 1.11-1.04\left(\mathrm{~m}, 3 \mathrm{H}, \mathrm{CH}_{3}\right) .{ }^{13} \mathrm{C}\left\{{ }^{1} \mathrm{H}\right\}$ $\operatorname{NMR}\left(\delta, \mathrm{d}_{6}-\mathrm{DMSO}, 20^{\circ} \mathrm{C}\right): 150.9,132.6,118.4,115.0,47.1,15.9$. ESI-MS: $[\mathrm{M}+\mathrm{H}]^{+}$, found 150.13. $\left[\mathrm{C}_{10} \mathrm{H}_{16} \mathrm{~N}\right]^{+}$requires 150.13, MS-MS: 122.20 .

N-Isopentylbenzeneamine (8)

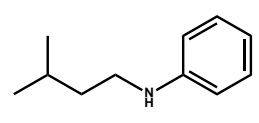

colourless oil $139 \mathrm{mg}(85 \%)$. 1H NMR ( $\delta, \mathrm{d}_{6}$-DMSO, 20 $\left.\mathrm{C}\right):$ 7.09-7.03 (m, 2H, PhH), 6.58$6.48(\mathrm{~m}, 3 \mathrm{H}, \mathrm{PhH}), 5.43(\mathrm{t}, \mathrm{J}=5.2 \mathrm{~Hz}, 1 \mathrm{H}, \mathrm{NH}), 3.04-2.96\left(\mathrm{~m}, 2 \mathrm{H}, \mathrm{CH}_{2}\right), 1.71$ (septet, J= 6.7 $\mathrm{Hz}, 2 \mathrm{H}, \mathrm{CH}), 1.50-1.41\left(\mathrm{~m}, 2 \mathrm{H}, \mathrm{CH}_{2}\right), 0.92\left(\mathrm{~d}, \mathrm{~J}=6.7 \mathrm{~Hz}, 6 \mathrm{H}, \mathrm{CH}_{3}\right) .{ }^{13} \mathrm{C}\left\{{ }^{1} \mathrm{H}\right\} \mathrm{NMR}\left(\delta, \mathrm{CD}_{2} \mathrm{Cl}_{2}\right.$, $\left.20^{\circ} \mathrm{C}\right): 150.8,130.6,117.0,113.6,42.8,39.5,27.2$, 24.3. ESI-MS: $[\mathrm{M}+\mathrm{H}]^{+}$, found 164.13 . $\left[\mathrm{C}_{11} \mathrm{H}_{18} \mathrm{~N}\right]^{+}$requires 164.14, MS-MS: 94.36, 71.68.

\section{N-Benzyl-4-methylbenzeneamine (9) ${ }^{9}$}

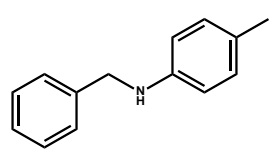

yellow oil $185 \mathrm{mg}(94 \%)^{\text {Table2, Entry4 }}, 177 \mathrm{mg}(90 \%)^{\text {Table3, Entry1 }}$. $1 \mathrm{H} \mathrm{NMR}\left(\delta, \mathrm{CD}_{2} \mathrm{Cl}_{2}, 20^{\circ} \mathrm{C}\right): 7.48-$ $7.39(\mathrm{~m}, 5 \mathrm{H}, \mathrm{PhH}), 7.09(\mathrm{~d}, \mathrm{~J}=8.1 \mathrm{~Hz}, 2 \mathrm{H}, \mathrm{PhH}), 6.65(\mathrm{~d}, \mathrm{~J}=8.2 \mathrm{~Hz}, 2 \mathrm{H}, \mathrm{PhH}), 4.40(\mathrm{~s}, 2 \mathrm{H}$, $\left.\mathrm{CH}_{2}\right), 4.10(\mathrm{~s}, 1 \mathrm{H}, \mathrm{NH}), 2.35\left(\mathrm{~s}, 3 \mathrm{H}, \mathrm{CH}_{3}\right) \cdot{ }^{13} \mathrm{C}\left\{{ }^{1} \mathrm{H}\right\} \mathrm{NMR}\left(\delta, \mathrm{CD}_{2} \mathrm{Cl}_{2}, 20^{\circ} \mathrm{C}\right): 147.4,141.4$, $131.0,129.9,128.8,127.9,127.3,114.3,49.7,21.5$. ESI-MS: $[M+H]^{+}$, found 198.04 . $\left[\mathrm{C}_{14} \mathrm{H}_{16} \mathrm{~N}\right]^{+}$requires 198.13, MS-MS: $120.20,106.26,91.38$.

N-(4-Fluorobenzyl)-4-methylbenzeneamine (10) ${ }^{10}$ )

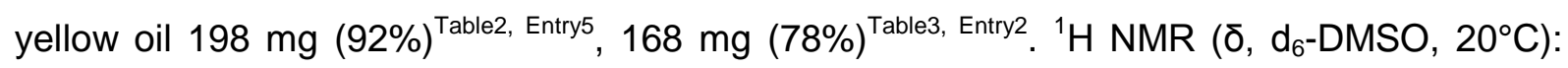
7.40-7.34 (m, 2H, PhH), 7.21-7.15 (m, 2H, PhH), $6.85(\mathrm{~d}, \mathrm{~J}=8.3 \mathrm{~Hz}, 2 \mathrm{H}, \mathrm{PhH}), 6.47$ (d, J= $\left.8.6 \mathrm{~Hz}, 2 \mathrm{H}, \mathrm{PhH}), 6.02(\mathrm{~s}, \mathrm{br}, 1 \mathrm{H}, \mathrm{NH}), 4.21\left(\mathrm{~s}, 2 \mathrm{H}, \mathrm{CH}_{2}\right), 2.11\left(\mathrm{~s}, 3 \mathrm{H}, \mathrm{CH}_{3}\right) .{ }^{13} \mathrm{C}^{1} \mathrm{H}\right\} \mathrm{NMR}(\delta$, $\mathrm{d}_{6}$-DMSO, 20 $\left.{ }^{\circ} \mathrm{C}\right): 162.9(\mathrm{~d}, \mathrm{~J}=243.0 \mathrm{~Hz}), 148.0,138.3(\mathrm{~d}, \mathrm{~J}=2.9 \mathrm{~Hz}), 131.0,130.7$ (d, J=8.2 $\mathrm{Hz}), 116.7(\mathrm{~d}, \mathrm{~J}=21.2 \mathrm{~Hz}), 114.2,47.7,21.8 .{ }^{19} \mathrm{~F}$ NMR $\left(\delta, \mathrm{d}_{6}-\mathrm{DMSO}, 20^{\circ} \mathrm{C}\right): 116.7$. ESI-MS: $[\mathrm{M}+\mathrm{H}]^{+}$, found 215.94. $\left[\mathrm{C}_{14} \mathrm{H}_{15} \mathrm{FN}\right]^{+}$requires 216.12, MS-MS: 109.06, 106.12. 
N-(4-Chlorobenzyl)-4-methylbenzeneamine (11) ${ }^{11}$

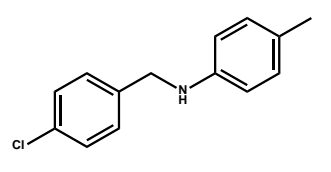

white solid $167 \mathrm{mg}(72 \%)^{\text {Table2, Entry6 }}, 153 \mathrm{mg}(66 \%)^{\text {Table3, Entry3 }} \cdot{ }^{1} \mathrm{H}$ NMR $\left(\delta, \mathrm{CD}_{2} \mathrm{Cl}_{2}, 20^{\circ} \mathrm{C}\right)$ : 7.35 (s, 5H, PhH), 7.01 (d, J= 8.2 Hz, 1H, PhH), 6.65 (d, J= $8.2 \mathrm{~Hz}, 2 \mathrm{H}, \mathrm{PhH}), 4.33(\mathrm{~s}, 2 \mathrm{H}$, $\mathrm{CH}_{2}$ ), 4.09 (s, $\left.1 \mathrm{H}, \mathrm{NH}\right), 2.25\left(\mathrm{~s}, 3 \mathrm{H}, \mathrm{CH}_{3}\right) .{ }^{13} \mathrm{C}\left\{{ }^{1} \mathrm{H}\right\} \mathrm{NMR}\left(\delta, \mathrm{CD}_{2} \mathrm{Cl}_{2}, 20^{\circ} \mathrm{C}\right): 147.0,140.0$, 133.8, 130.9, 130.0, 129.8, 128.1, 114.2, 48.9, 21.3. ESI-MS: $[\mathrm{M}+\mathrm{H}]^{+}$, found $231.96(\mathrm{Cl}-$ Isotope: 3:1; 233.91). [ $\left.\mathrm{C}_{14} \mathrm{H}_{15} \mathrm{CIN}\right]^{+}$requires 232.09, MS-MS: 127.08, 125.12, 106.27.

\section{$\mathrm{N}$-(3,4-Dimethoxybenzyl)benzeneamine (12) ${ }^{12}$}

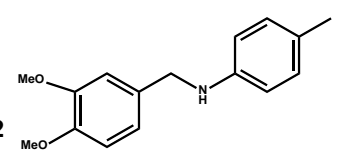

white solid $193 \mathrm{mg}(75 \%) .{ }^{1} \mathrm{H}$ NMR ( $\delta, \mathrm{d}_{6}$-DMSO, 20 $\left.{ }^{\circ} \mathrm{C}\right): 6.98(\mathrm{~s}, 1 \mathrm{H}, \mathrm{PhH}), 6.87-6.85(\mathrm{~m}, 4 \mathrm{H}$, $\mathrm{PhH}), 6.51(\mathrm{~d}, \mathrm{~J}=8.2 \mathrm{~Hz}, 2 \mathrm{H}, \mathrm{PhH}), 5.88(\mathrm{t}, \mathrm{J}=6.0 \mathrm{~Hz}, 1 \mathrm{H}, \mathrm{NH}), 4.15\left(\mathrm{~d}, \mathrm{~J}=5.9 \mathrm{~Hz}, 2 \mathrm{H}, \mathrm{CH}_{2}\right.$ ), $3.73\left(\mathrm{~s}, 3 \mathrm{H}, \mathrm{CH}_{3}\right), 3.72\left(\mathrm{~s}, 3 \mathrm{H}, \mathrm{CH}_{3}\right), 2.13\left(\mathrm{~s}, 3 \mathrm{H}, \mathrm{CH}_{3}\right) .{ }^{13} \mathrm{C}\left\{{ }^{1} \mathrm{H}\right\} \operatorname{NMR}\left(\delta, \mathrm{d}_{6}-\mathrm{DMSO}, 20^{\circ} \mathrm{C}\right)$ : $149.2,148.1,147.0,133.3,129.7,124.5,119.7,113.0,112.2,111.7,56.0,55.9,47.1,20.5$. ESI-MS: $[\mathrm{M}+\mathrm{H}]^{+}$, found 257.98. $\left[\mathrm{C}_{16} \mathrm{H}_{20} \mathrm{NO}_{2}\right]^{+}$requires 258.15, MS-MS: 151.06 .

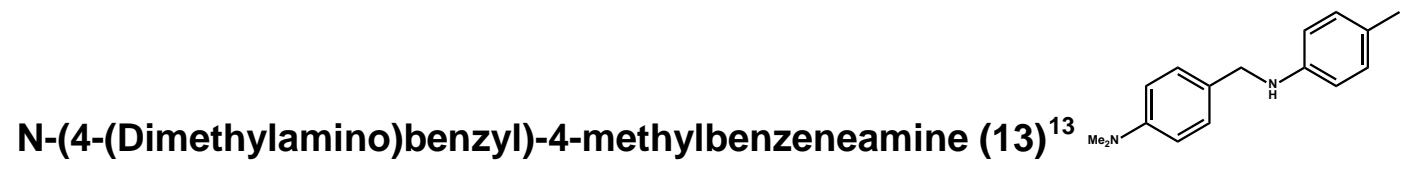
colourless crystals $208 \mathrm{mg}(87 \%)^{\text {Table2, Entry8 }}, 191 \mathrm{mg}(80 \%)^{\text {Table3, Entry4 }}{ }^{1} \mathrm{H}$ NMR $\left(\delta, \mathrm{d}_{6}\right.$-DMSO): $7.15(\mathrm{~d}, \mathrm{~J}=8.5 \mathrm{~Hz}, 2 \mathrm{H}, \mathrm{PhH}), 6.83(\mathrm{~d}, \mathrm{~J}=8.0 \mathrm{~Hz}, 2 \mathrm{H}, \mathrm{PhH}), 6.67$ (d, J=8.0 Hz, 2H, PhH), $6.47(\mathrm{~d}, \mathrm{~J}=8.1 \mathrm{~Hz}, 2 \mathrm{H}, \mathrm{PhH}), 5.78(\mathrm{t}, \mathrm{J}=6.3 \mathrm{~Hz}, 1 \mathrm{H}, \mathrm{NH}), 4.08\left(\mathrm{~d}, \mathrm{~J}=4.2 \mathrm{~Hz}, 2 \mathrm{H}, \mathrm{CH}_{2}\right), 2.84$ $\left(\mathrm{s}, 6 \mathrm{H}, \mathrm{CH}_{3}\right), 2.12\left(\mathrm{~s}, 3 \mathrm{H}, \mathrm{CH}_{3}\right) \cdot{ }^{13} \mathrm{C}\left\{{ }^{1} \mathrm{H}\right\} \operatorname{NMR}\left(\delta, \mathrm{d}_{6}-\mathrm{DMSO}, 20^{\circ} \mathrm{C}\right): 151.2,148.4,130.9$, 129.5, 125.5, 114.2, 48.1, 42.1, 21.8. ESI-MS: $[\mathrm{M}+\mathrm{H}]^{+}$, found 241.05. $\left[\mathrm{C}_{16} \mathrm{H}_{21} \mathrm{~N}_{2}\right]^{+}$requires 241.17, MS-MS: 134.16, 120.08.

\section{$\mathrm{N}-\left(\left(\right.\right.$ Benzo[d][1,3]dioxol-6-yl)methyl)benzeneamine (14) ${ }^{14}$}

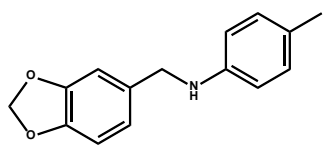

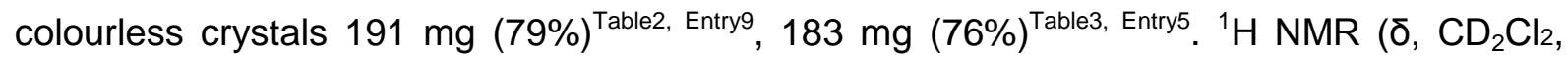
$\left.20^{\circ} \mathrm{C}\right)$ : 6.98-6.82 (m, 5H, PhH), $6.76(\mathrm{~d}, \mathrm{~J}=8.5 \mathrm{~Hz}, 2 \mathrm{H}, \mathrm{PhH}), 5.93\left(\mathrm{~s}, 2 \mathrm{H}, \mathrm{CH}_{2}\right), 4.20(\mathrm{~s}, 2 \mathrm{H}$, $\mathrm{CH}_{2}$ ), 3.97 (s, br, $\left.1 \mathrm{H}, \mathrm{NH}\right), 2.21\left(\mathrm{~s}, 3 \mathrm{H}, \mathrm{CH}_{3}\right) \cdot{ }^{13} \mathrm{C}\left\{{ }^{1} \mathrm{H}\right\} \mathrm{NMR}\left(\delta, \mathrm{CD}_{2} \mathrm{Cl}_{2}, 20^{\circ} \mathrm{C}\right): 149.2,147.9$, 147.2, 135.2, 130.9, 127.9, 121.7, 114.2, 109.4, 109.1, 102.4, 49.4, 21.4. ESI-MS: $[\mathrm{M}+\mathrm{H}]^{+}$, found 241.98. $\left[\mathrm{C}_{15} \mathrm{H}_{16} \mathrm{NO}_{2}\right]^{+}$requires 242.18, MS-MS: 135.12. 


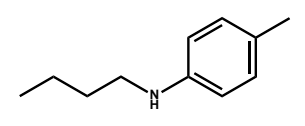

red oil $145 \mathrm{mg}(89 \%)^{\text {Table2, Entry10 }}, 145 \mathrm{mg}(89 \%)^{\text {Table3, Entry6 }}{ }^{1} \mathrm{H}$ NMR $\left(\delta, \mathrm{CD}_{2} \mathrm{Cl}_{2}, 20^{\circ} \mathrm{C}\right): 7.07$ (d, $\mathrm{J}=7.5 \mathrm{~Hz}, 2 \mathrm{H}, \mathrm{PhH}), 6.60(\mathrm{~d}, \mathrm{~J}=8.3 \mathrm{~Hz}, 2 \mathrm{H}, \mathrm{PhH}), 3.16(\mathrm{t}, \mathrm{J}=6.9 \mathrm{~Hz}, 1 \mathrm{H}, \mathrm{NH}), 2.32(\mathrm{~s}, 3 \mathrm{H}$, $\left.\mathrm{CH}_{3}\right), 1.73-1.48\left(\mathrm{~m}, 4 \mathrm{H}, \mathrm{CH}_{2}\right), 1.06\left(\mathrm{t}, \mathrm{J}=7.1 \mathrm{~Hz}, 3 \mathrm{H}, \mathrm{CH}_{3}\right) .{ }^{13} \mathrm{C}\left\{{ }^{1} \mathrm{H}\right\} \operatorname{NMR}\left(\delta, \mathrm{CD}_{2} \mathrm{Cl}_{2}, 20^{\circ} \mathrm{C}\right)$ : 149.6, 132.7, 129.0, 115.8, 47.0, 34.9, 23.4, 23.2, 16.9. ESI-MS: $[\mathrm{M}+\mathrm{H}]^{+}$, found 164.20 . $\left[\mathrm{C}_{11} \mathrm{H}_{18} \mathrm{~N}\right]^{+}$requires 164.14, MS-MS: 120.08, 108.08.

\section{4-Methyl-N-((pyridine-2-yl)methyl)benzeneamine $(17)^{16}$}

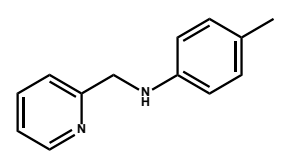

orange solid $32 \mathrm{mg}(17 \%) .{ }^{1} \mathrm{H}$ NMR $\left(\delta, \mathrm{CD}_{2} \mathrm{Cl}_{2}, 20^{\circ} \mathrm{C}\right): 8.63(\mathrm{~d}, \mathrm{~J}=4.1 \mathrm{~Hz}, 1 \mathrm{H}, \mathrm{PyH}), 7.72-$ $7.65(\mathrm{~m}, 1 \mathrm{H}, \mathrm{PyH}), 7.25$ (d, J= 8.2 Hz, 1H, PyH), 7.25-7.20 (m, 1H, PyH), $7.22(\mathrm{~d}, \mathrm{~J}=6.8 \mathrm{~Hz}$, $2 \mathrm{H}, \mathrm{PhH}), 6.63(\mathrm{~d}, \mathrm{~J}=8.8 \mathrm{~Hz}, 2 \mathrm{H}, \mathrm{PhH}), 4.82(\mathrm{~s}, \mathrm{br}, 1 \mathrm{H}, \mathrm{NH}), 4.46\left(\mathrm{~s}, 2 \mathrm{H}, \mathrm{CH}_{2}\right), 2.28(\mathrm{~s}, 3 \mathrm{H}$, $\left.\mathrm{CH}_{3}\right) .{ }^{13} \mathrm{C}\left\{{ }^{1} \mathrm{H}\right\} \operatorname{NMR}\left(\delta, \mathrm{CD}_{2} \mathrm{Cl}_{2}, 20^{\circ} \mathrm{C}\right): 160.2,150.4,147.2,137.7,130.9,127.8,123.3,122.8$, 114.3, 50.7, 21.4. ESI-MS: $[\mathrm{M}+\mathrm{H}]^{+}$, found 199.06. $\left[\mathrm{C}_{13} \mathrm{H}_{15} \mathrm{~N}_{2}\right]^{+}$requires 199.13, MS-MS: $182.05,107.27$.

\section{$\mathrm{N}$-((Thiophene-2-yl)methyl)-4-methylbenzeneamine (18) ${ }^{17}$}

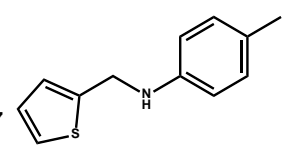

yellow oil $120 \mathrm{mg}(59 \%) .{ }^{1} \mathrm{H}$ NMR $\left(\delta, \mathrm{d}_{6}\right.$-DMSO, 20 $\left.{ }^{\circ} \mathrm{C}\right): 7.34(\mathrm{~d}, \mathrm{~J}=7.3 \mathrm{~Hz}, 1 \mathrm{H}, \mathrm{ThH}), 7.03(\mathrm{~s}$, br, $1 \mathrm{H}, \mathrm{ThH}), 6.98-6.89(\mathrm{~m}, 3 \mathrm{H}, \mathrm{PhH}, \mathrm{ThH}), 6.58(\mathrm{~d}, \mathrm{~J}=6.6 \mathrm{~Hz}, 2 \mathrm{H}, \mathrm{PhH}), 6.01(\mathrm{~s}, \mathrm{br}, 1 \mathrm{H}, \mathrm{NH})$ $4.43\left(\mathrm{~s}, 2 \mathrm{H}, \mathrm{CH}_{2}\right), 2.16\left(\mathrm{~s}, 3 \mathrm{H}, \mathrm{CH}_{3}\right) .{ }^{13} \mathrm{C}\left\{{ }^{1} \mathrm{H}\right\} \operatorname{NMR}\left(\delta, \mathrm{d}_{6}-\mathrm{DMSO}, 20^{\circ} \mathrm{C}\right.$ ): 146.5, 145.2, 129.8, 127.2, 125.1, 125.0, 124.8, 113.2, 42.8, 20.6. ESI-MS: $[\mathrm{M}+\mathrm{H}]^{+}$, found 204.00. $\left[\mathrm{C}_{12} \mathrm{H}_{14} \mathrm{NS}\right]^{+}$ requires 204.08, MS-MS: 106.27, 97.28 .

\section{$\mathrm{N}$-((Furane-2-yl)methyl)-4-methylbenzeneamine (19) ${ }^{18}$}

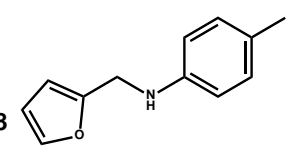

yellow oil $118 \mathrm{mg}(63 \%) .{ }^{1} \mathrm{H} \operatorname{NMR}\left(\delta, \mathrm{CD}_{2} \mathrm{Cl}_{2}, 20^{\circ} \mathrm{C}\right): 7.50(\mathrm{dd}, \mathrm{J}=0.9 \mathrm{~Hz}, \mathrm{~J}=1.9 \mathrm{~Hz}, 1 \mathrm{H}$, FuH), $6.72(\mathrm{~d}, \mathrm{~J}=8.0 \mathrm{~Hz}, 2 \mathrm{H}, \mathrm{PhH}), 6.69(\mathrm{~d}, \mathrm{~J}=8.6 \mathrm{~Hz}, 2 \mathrm{H}, \mathrm{PhH}), 6.47$ (dd, J= $1.9 \mathrm{~Hz}, \mathrm{~J}=3.1$ $\mathrm{Hz}, 1 \mathrm{H}, \mathrm{FuH}), 6.35$ (dd, J=0.9 Hz, J= $3.1 \mathrm{~Hz}, 1 \mathrm{H}, \mathrm{FuH}), 4.39\left(\mathrm{~s}, 2 \mathrm{H}, \mathrm{CH}_{2}\right), 4.07(\mathrm{~s}, \mathrm{br}, 1 \mathrm{H}$, $\mathrm{NH}), 2.37$ (s, 3H, $\left.\mathrm{CH}_{3}\right) .{ }^{13} \mathrm{C}\left\{{ }^{1} \mathrm{H}\right\} \operatorname{NMR}\left(\delta, \mathrm{CD}_{2} \mathrm{Cl}_{2}, 20^{\circ} \mathrm{C}\right): 154.7,146.8,143.2,131.0,128.4$, 114.6, 111.7, 108.2, 42.9, 21.5. ESI-MS: $[\mathrm{M}+\mathrm{H}]^{+}$, found 187.94. $\left[\mathrm{C}_{12} \mathrm{H}_{14} \mathrm{NO}^{+}\right.$requires 188.11, MS-MS: 106.11, 84.29. 


\section{4-Methyl-N-((quinoline-4-yl)methyl)benzeneamine (20)}

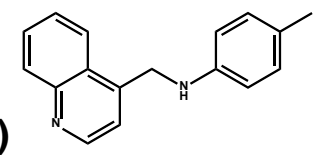

white solid $201 \mathrm{mg}(81 \%) .{ }^{1} \mathrm{H}$ NMR $\left(\delta, \mathrm{d}_{6}-\mathrm{DMSO}, 20^{\circ} \mathrm{C}\right): 8.80(\mathrm{~d}, \mathrm{~J}=4.1 \mathrm{~Hz}, 1 \mathrm{H}, \mathrm{PhH}), 8.23$ (d, J= $7.5 \mathrm{~Hz}, 1 \mathrm{H}, \mathrm{PhH}), 8.07$ (d, J= $8.5 \mathrm{~Hz}, 1 \mathrm{H}, \mathrm{PhH}), 7.80(\mathrm{t}, \mathrm{J}=8.5 \mathrm{~Hz}, 1 \mathrm{H}, \mathrm{PhH}), 7.74(\mathrm{t}$, $\mathrm{J}=7.5 \mathrm{~Hz}, 1 \mathrm{H}, \mathrm{PhH}), 7.65(\mathrm{~d}, \mathrm{~J}=3.4 \mathrm{~Hz}, 1 \mathrm{H}, \mathrm{PhH}), 7.44(\mathrm{~d}, \mathrm{~J}=4.1 \mathrm{~Hz}, 1 \mathrm{H}, \mathrm{PhH}), 6.87(\mathrm{~d}, \mathrm{~J}=$ $7.5 \mathrm{~Hz}, 1 \mathrm{H}, \mathrm{PhH}), 6.52(\mathrm{~d}, \mathrm{~J}=7.5 \mathrm{~Hz}, 1 \mathrm{H}, \mathrm{NH}), 4.77\left(\mathrm{~d}, \mathrm{~J}=4.8 \mathrm{~Hz}, 2 \mathrm{H}, \mathrm{CH}_{2}\right), 2.12(\mathrm{~s}, 3 \mathrm{H}$, $\left.\mathrm{CH}_{3}\right) .{ }^{13} \mathrm{C}\left\{{ }^{1} \mathrm{H}\right\} \operatorname{NMR}\left(\delta, \mathrm{d}_{6}\right.$-DMSO, $\left.20^{\circ} \mathrm{C}\right)$ ): 153.8, 151.2, 149.7, 149.1, 133.1, 132.9, 132.6, $129.9,129.9,128.0,127.2,122.6,115.8,47.1,23.6$. ESI-MS: $[M+H]^{+}$, found 249.02 . $\left[\mathrm{C}_{17} \mathrm{H}_{17} \mathrm{~N}_{2}\right]^{+}$requires 249.1392, HRMS (ESI): $[\mathrm{M}+\mathrm{H}]^{+}$, found 249.1386.

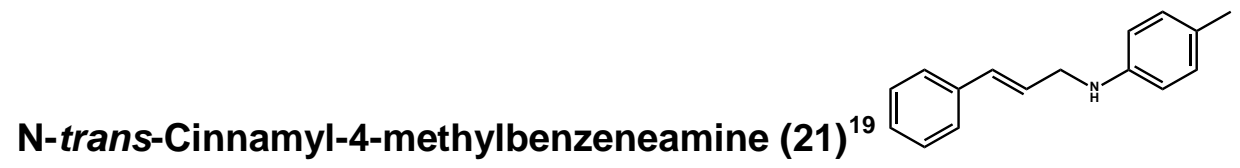

yellow solid $203 \mathrm{mg}(93 \%) .{ }^{1} \mathrm{H}$ NMR $\left(\delta, \mathrm{CD}_{2} \mathrm{Cl}_{2}, 20^{\circ} \mathrm{C}\right): 7.48-7.29(\mathrm{~m}, 5 \mathrm{H}, \mathrm{PhH}), 7.07(\mathrm{~d}, \mathrm{~J}=$ 8.0 Hz, 2H, PhH), 6.72-6.64 (m, 3H, NH, PhH), 6.47-6.36 (m, 1H, Cㅡ= $=$ C), $3.97(\mathrm{~d}, \mathrm{~J}=5.2 \mathrm{~Hz}$, $\left.2 \mathrm{H}, \mathrm{CH}_{2}\right), 2.31\left(\mathrm{~s}, 3 \mathrm{H}, \mathrm{CH}_{3}\right) \cdot{ }^{13} \mathrm{C}\left\{{ }^{1} \mathrm{H}\right\} \operatorname{NMR}\left(\delta, \mathrm{CD}_{2} \mathrm{Cl}_{2}, 20^{\circ} \mathrm{C}\right): 147.4,138.3,132.4,131.0$, $129.9,128.9,128.8,127.9,127.6,114.4,47.7,21.4$. ESI-MS: $[\mathrm{M}+\mathrm{H}]^{+}$, found 224.03 . $\left[\mathrm{C}_{16} \mathrm{H}_{18} \mathrm{~N}\right]^{+}$requires 224.14, MS-MS: 117.21, 106.28.

\section{$\mathrm{N}-(R)$-Citronellyl-4-methylbenzeneamine (22)}

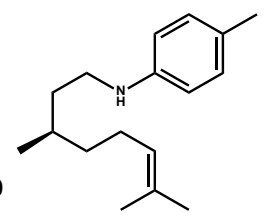

yellow oil $223 \mathrm{mg}(91 \%) .{ }^{1} \mathrm{H}$ NMR ( $\delta, \mathrm{d}_{6}$-DMSO, $20^{\circ} \mathrm{C}$ ): 6.87 (d, J=8.2 Hz, 2H, PhH), 6.46 (d, $\mathrm{J}=8.4 \mathrm{~Hz}, 2 \mathrm{H}, \mathrm{PhH}), 5.18-5.08(\mathrm{~m}, 2 \mathrm{H}, \mathrm{NH}, \mathrm{C} \underline{\mathrm{H}}=\mathrm{C}), 3.05-2.91\left(\mathrm{~m}, 2 \mathrm{H}, \mathrm{CH}_{2}\right), 2.15(\mathrm{~s}, 3 \mathrm{H}$, $\left.\mathrm{CH}_{3}\right), 2.00-1.92\left(\mathrm{~m}, 2 \mathrm{H}, \mathrm{CH}_{2}\right), 1.66\left(\mathrm{~s}, 3 \mathrm{H}, \mathrm{CH}_{3}\right), 1.58\left(\mathrm{~s}, 3 \mathrm{H}, \mathrm{CH}_{3}\right), 1.37-1.28\left(\mathrm{~m}, 2 \mathrm{H}, \mathrm{CH}_{2}\right)$, 1.21-1.11 (m, 1H, CH), $0.91\left(\mathrm{~d}, \mathrm{~J}=6.4 \mathrm{~Hz}, 3 \mathrm{H}, \mathrm{CH}_{3}\right) .{ }^{13} \mathrm{C}\left\{{ }^{1} \mathrm{H}\right\}$ NMR $\left(\delta, \mathrm{d}_{6}-\mathrm{DMSO}, 20^{\circ} \mathrm{C}\right)$ : 148.6, 132.1, 131.2, 130.9, 126.4, 126.2, 125.3, 113.8, 42.9, 38.5, 37.7, 31.7, 27.2, 26.8, 21.8, 21.1, 19.2. ESI-MS: $[\mathrm{M}+\mathrm{H}]^{+}$, found 246.04. $\left[\mathrm{C}_{17} \mathrm{H}_{28} \mathrm{~N}\right]^{+}$requires 246.2222, HRMS (ESI): $[\mathrm{M}+\mathrm{H}]^{+}$, found 246.2216 .

\section{N-((1H-Indol-3-yl)methyl)-4-methylbenzeneamine (23)}

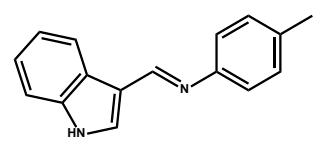

slightly yellow solid $186 \mathrm{mg}(79 \%) .{ }^{1} \mathrm{H}$ NMR $\left(\delta, \mathrm{d}_{6}-\mathrm{DMSO}, 20^{\circ} \mathrm{C}\right): 10.89(\mathrm{~s}, 1 \mathrm{H}, \mathrm{NH}), 7.63(\mathrm{~d}$, $\mathrm{J}=8.7 \mathrm{~Hz}, 1 \mathrm{H}, \mathrm{PhH}), 7.37(\mathrm{dd}, \mathrm{J}=0.7 \mathrm{~Hz}, \mathrm{~J}=7.9 \mathrm{~Hz}, 1 \mathrm{H}, \mathrm{PhH}), 7.29(\mathrm{~d}, \mathrm{~J}=2.1 \mathrm{~Hz}, 1 \mathrm{H}, \mathrm{PhH})$, 7.12-6.96 (m, 2H, PhH), $6.87(\mathrm{~d}, \mathrm{~J}=8.1 \mathrm{~Hz}, 2 \mathrm{H}, \mathrm{PhH}), 6.60(\mathrm{~d}, \mathrm{~J}=8.4 \mathrm{~Hz}, 2 \mathrm{H}, \mathrm{PhH}), 5.61(\mathrm{~s}$, 
$1 \mathrm{H}, \mathrm{NH}), 4.34\left(\mathrm{~s}, 2 \mathrm{H}, \mathrm{CH}_{2}\right), 2.14\left(\mathrm{~s}, 3 \mathrm{H}, \mathrm{CH}_{3}\right) .{ }^{13} \mathrm{C}\left\{{ }^{1} \mathrm{H}\right\}$ NMR $\left(\delta, \mathrm{d}_{6}-\mathrm{DMSO}, 20^{\circ} \mathrm{C}\right): 150.4$, $139.9,132.7,130.2,127.3,127.1,124.5,122.3,121.9,116.3,115.9,114.9,42.6$, 23.6. ESIMS: $[\mathrm{M}+\mathrm{H}]^{+}$, found $235.01\left[\mathrm{C}_{16} \mathrm{H}_{15} \mathrm{~N}_{2}\right]^{+}$(dehydrogenation). $\left[\mathrm{C}_{16} \mathrm{H}_{17} \mathrm{~N}_{2}\right]^{+}$requires 237.1392. HRMS (ESI): $[\mathrm{M}+\mathrm{H}]^{+}$, found 235.1230 (dehydrogenation).

\section{N-Benzyl-5-chloro-2,4-dimethoxybenzeneamine (24)}

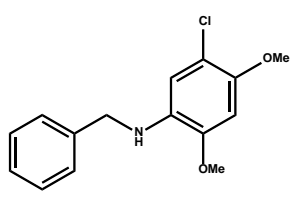

grey crystals $200 \mathrm{mg}(72 \%) .{ }^{1} \mathrm{H}$ NMR $\left(\delta, \mathrm{d}_{6}-\mathrm{DMSO}, 20^{\circ} \mathrm{C}\right): 7.34-7.20(\mathrm{~m}, 5 \mathrm{H}, \mathrm{PhH}), 6.75(\mathrm{~s}$, $1 \mathrm{H}, \mathrm{PhH}), 6.39(\mathrm{~s}, 1 \mathrm{H}, \mathrm{PhH}), 5.41(\mathrm{t}, \mathrm{J}=6.0 \mathrm{~Hz}, 1 \mathrm{H}, \mathrm{NH}), 4.29\left(\mathrm{~d}, \mathrm{~J}=5.9 \mathrm{~Hz}, 2 \mathrm{H}, \mathrm{CH}_{2}\right), 3.86$ $\left(\mathrm{s}, 3 \mathrm{H}, \mathrm{CH}_{3}\right), 3.77\left(\mathrm{~s}, 3 \mathrm{H}, \mathrm{CH}_{3}\right) .{ }^{13} \mathrm{C}\left\{{ }^{1} \mathrm{H}\right\} \operatorname{NMR}\left(\delta, \mathrm{d}_{6}-\mathrm{DMSO}, 20^{\circ} \mathrm{C}\right): 147.8,147.1,141.9$, $134.8,130.1,128.7,128.4,114.1,112.0,101.0,58.8,57.7,48.3$. ESI-MS: $[\mathrm{M}+\mathrm{H}]^{+}$, found 277.95 (Cl-Isotope: 3:1; 279.90). $\left[\mathrm{C}_{15} \mathrm{H}_{17} \mathrm{CINO}_{2}\right]^{+}$requires 278.0948, HRMS (ESI): $[\mathrm{M}+\mathrm{H}]^{+}$, found 278.0942 .

\section{N-benzyl-4-methoxybenzeneamine (25) ${ }^{21}$}

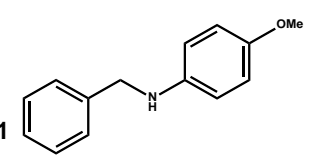

brown crystals $192 \mathrm{mg}(90 \%) .{ }^{1} \mathrm{H}$ NMR ( $\delta, \mathrm{d}_{6}$-DMSO, 20 $\left.\mathrm{C}\right):$ 7.38-7.19 (m, 5H, PhH), 6.69 (d, $\mathrm{J}=8.9 \mathrm{~Hz}, 2 \mathrm{H}, \mathrm{PhH}), 6.52(\mathrm{~d}, \mathrm{~J}=8.9 \mathrm{~Hz}, 2 \mathrm{H}, \mathrm{PhH}), 5.81(\mathrm{t}, \mathrm{J}=6.5 \mathrm{~Hz}, 1 \mathrm{H}, \mathrm{NH}), 4.20(\mathrm{~d}, \mathrm{~J}=$ $\left.6.7 \mathrm{~Hz}, 2 \mathrm{H}, \mathrm{CH}_{2}\right), 3.62\left(\mathrm{~s}, 3 \mathrm{H}, \mathrm{CH}_{3}\right) .{ }^{13} \mathrm{C}\left\{{ }^{1} \mathrm{H}\right\}$ NMR (ס, $\left.\mathrm{d}_{6}-\mathrm{DMSO}, 20^{\circ} \mathrm{C}\right): 152.4,144.7,142.3$, 130.0, 129.0, 128.3, 116.3, 115.1, 57.0, 49.0. ESI-MS: $[\mathrm{M}+\mathrm{H}]^{+}$, found 214.02. $\left[\mathrm{C}_{14} \mathrm{H}_{16} \mathrm{NO}\right]^{+}$ requires 214.12, MS-MS: 136.13, 123.19, 108.27, 91.40.

$\mathrm{N}$-(4-Fluorobenzyl)pyridine-2-amine (26) ${ }^{26}$,

colourless crystals $46 \mathrm{mg}(23 \%) .{ }^{1} \mathrm{H}$ NMR $\left(\delta, \mathrm{CD}_{2} \mathrm{Cl}_{2}, 20^{\circ} \mathrm{C}\right): 8.09$ (dd, J=1.1Hz, J=5.0 Hz, $1 \mathrm{H}, \mathrm{PhH}), 7.46-7.36(\mathrm{~m}, 3 \mathrm{H}, \mathrm{PhH}), 7.06(\mathrm{t}, \mathrm{J}=8.8 \mathrm{~Hz}, 2 \mathrm{H}, \mathrm{PyH}), 6.63-6.60(\mathrm{~m}, 2 \mathrm{H}, \mathrm{PhH}), 6.43$ $(\mathrm{d}, \mathrm{J}=8.4 \mathrm{~Hz}, 1 \mathrm{H}, \mathrm{PhH}), 5.12(\mathrm{~s}, \mathrm{br}, 1 \mathrm{H}, \mathrm{NH}), 4.52\left(\mathrm{~d}, \mathrm{~J}=5.8 \mathrm{~Hz}, 2 \mathrm{H}, \mathrm{CH}_{2}\right) .{ }^{13} \mathrm{C}\left\{{ }^{1} \mathrm{H}\right\} \mathrm{NMR}(\delta$, $\mathrm{CD}_{2} \mathrm{Cl}_{2}, 20^{\circ} \mathrm{C}$ ): $147.0,140.0,133.8,130.9,130.0,129.8,128.1,114.2,48.9,21.3$. ESI-MS: $[\mathrm{M}+\mathrm{H}]^{+}$, found 203.09. $\left[\mathrm{C}_{12} \mathrm{H}_{12} \mathrm{FN}_{2}\right]^{+}$requires 203.10, MS-MS: 109.04 .

N-Benzylnaphthenamine (27) ${ }^{22}$ (1)

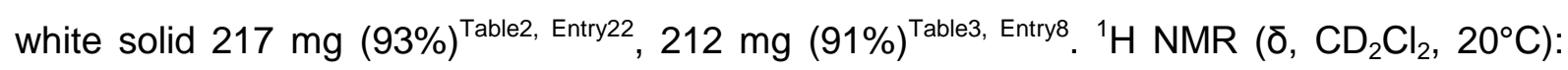
7.73-7.20 (m, 10H, PhH), 7.01-6.88 (m, 2H, PhH), 4.49 (d, J=5.4 Hz, 2H, $\left.\mathrm{CH}_{2}\right), 4.39$ (s, br, 
$1 \mathrm{H}, \mathrm{NH}) .{ }^{13} \mathrm{C}\left\{{ }^{1} \mathrm{H}\right\} \operatorname{NMR}\left(\delta, \mathrm{CD}_{2} \mathrm{Cl}_{2}, 20^{\circ} \mathrm{C}\right): 164.0,159.1,111.2,42.4,33.0,21.5,14.9 . \mathrm{ESI}-$ MS: $[\mathrm{M}+\mathrm{H}]^{+}$, found 234.00. $\left[\mathrm{C}_{17} \mathrm{H}_{16} \mathrm{~N}\right]^{+}$requires 234.13, MS-MS: 156.06, 143.11, 91.38.

N-Butyl-3-aminopyridine (28) ${ }^{23}$

colourless oil $117 \mathrm{mg}(78 \%) .{ }^{1} \mathrm{H}$ NMR $\left(\delta, \mathrm{CD}_{2} \mathrm{Cl}_{2}, 20^{\circ} \mathrm{C}\right): 8.03(\mathrm{~d}, \mathrm{~J}=2.7 \mathrm{~Hz}, 1 \mathrm{H}, \mathrm{PyH}), 7.09$ (d, J= 4.6 Hz, 1H, PyH), 7.12-7.06 (m, 1H, PyH), 6.92-6.87 (m, 1H, PyH), 4.33 (s, br, 1H, $\mathrm{NH}$ ), $6.13\left(\mathrm{t}, \mathrm{J}=6.9 \mathrm{~Hz}, 2 \mathrm{H}, \mathrm{CH}_{2}\right.$ ), 1.63 (quintet, J= $7.0 \mathrm{~Hz}, 2 \mathrm{H}, \mathrm{CH}_{2}$ ), 1.47 (quintet, $\mathrm{J}=7.1 \mathrm{~Hz}$, $\left.2 \mathrm{H}, \mathrm{CH}_{2}\right), 0.98\left(\mathrm{t}, \mathrm{J}=7.2 \mathrm{~Hz}, 3 \mathrm{H}, \mathrm{CH}_{3}\right) \cdot{ }^{13} \mathrm{C}\left\{{ }^{1} \mathrm{H}\right\} \operatorname{NMR}\left(\delta, \mathrm{CD}_{2} \mathrm{Cl}_{2}, 20^{\circ} \mathrm{C}\right): 147.9,140.8,138.7$, 126.7, 121.0, 46.2, 34.4, 23.3, 16.7. ESI-MS: $[\mathrm{M}+\mathrm{H}]^{+}$, found 151.14. $\left[\mathrm{C}_{9} \mathrm{H}_{15} \mathrm{~N}_{2}\right]^{+}$requires 151.12, MS-MS: 134.17, 82.51.

N-Benzyl-3-aminopyridine (29) ${ }^{24}$

colourless solid $155 \mathrm{mg}(84 \%)^{\text {Table2, Entry29 }}, 134 \mathrm{mg}(73 \%)^{\text {Table3, Entry }} .{ }^{1} \mathrm{H}$ NMR $\left(\delta, \mathrm{CD}_{2} \mathrm{Cl}_{2}\right.$, $\left.20^{\circ} \mathrm{C}\right): 8.17(\mathrm{~d}, \mathrm{~J}=2.9 \mathrm{~Hz}, 1 \mathrm{H}, \mathrm{PyH}), 8.01(\mathrm{dd}, \mathrm{J}=4.5 \mathrm{~Hz}, \mathrm{~J}=1.1 \mathrm{~Hz}, 1 \mathrm{H}, \mathrm{PyH}), 7.47-7.36(\mathrm{~m}$, $5 \mathrm{H}, \mathrm{PhH}), 7.15-7.09(\mathrm{~m}, 1 \mathrm{H}, \mathrm{PyH}), 6.98-6.93(\mathrm{~m}, 1 \mathrm{H}, \mathrm{PyH}), 5.23(\mathrm{~s}, \mathrm{br}, 1 \mathrm{H}, \mathrm{NH}), 4.40$ (d, J= $\left.5.3 \mathrm{~Hz}, 2 \mathrm{H}, \mathrm{CH}_{2}\right) \cdot{ }^{13} \mathrm{C}\left\{{ }^{1} \mathrm{H}\right\} \mathrm{NMR}\left(\delta, \mathrm{CD}_{2} \mathrm{Cl}_{2}, 20^{\circ} \mathrm{C}\right): 147.7,142.3,139.2,131.8,130.5,130.4$, 126.9, 121.5, 50.6. ESI-MS: $[\mathrm{M}+\mathrm{H}]^{+}$, found 185.04. $\left[\mathrm{C}_{12} \mathrm{H}_{13} \mathrm{~N}_{2}\right]^{+}$requires 185.11, MS-MS: $106.27,91.38$

N-Butyl-2-aminopyrimidine (30) ${ }^{25}$

colourless liquid $113 \mathrm{mg}(78 \%) .{ }^{1} \mathrm{H}$ NMR $\left(\delta, \mathrm{CD}_{2} \mathrm{Cl}_{2}, 20^{\circ} \mathrm{C}\right): 8.26(\mathrm{~d}, \mathrm{~J}=4.8 \mathrm{~Hz}, 2 \mathrm{H}, \mathrm{PhH})$, $6.51(\mathrm{t}, \mathrm{J}=4.7 \mathrm{~Hz}, 1 \mathrm{H}, \mathrm{PhH}), 6.8(\mathrm{~s}, \mathrm{br}, 1 \mathrm{H}, \mathrm{NH}), 3.42-3.37\left(\mathrm{~m}, 2 \mathrm{H}, \mathrm{CH}_{2}\right), 1.60$ (quintet, J= 7.3 $\mathrm{Hz}, 2 \mathrm{H}, \mathrm{CH}_{2}$ ), 1.43 (quintet, $\mathrm{J}=7.2 \mathrm{~Hz}, 2 \mathrm{H}, \mathrm{CH}_{2}$ ), $\left.0.95\left(\mathrm{t}, \mathrm{J}=7.2 \mathrm{~Hz}, 3 \mathrm{H}, \mathrm{CH}_{3}\right) .{ }^{13} \mathrm{C}^{1} \mathrm{H}\right\} \mathrm{NMR}$ $\left(\delta, \mathrm{CD}_{2} \mathrm{Cl}_{2}, 20^{\circ} \mathrm{C}\right): 164.0,159.1,111.2,42.4,33.0,21.5,14.9$. ESI-MS: $[\mathrm{M}+\mathrm{H}]^{+}$, found 152.38. $\left[\mathrm{C}_{8} \mathrm{H}_{14} \mathrm{~N}_{3}\right]^{+}$requires 152.12, MS-MS: 96.38 .

N-Benzyl-2-aminopyrimidine (31) $\mathbf{2}^{26}$

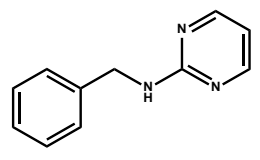

yellow oil $148 \mathrm{mg}(80 \%) .{ }^{1} \mathrm{H}$ NMR ( $\delta, \mathrm{d}_{6}$-DMSO, 20 $\left.{ }^{\circ} \mathrm{C}\right): 8.26(\mathrm{~d}, \mathrm{~J}=4.9 \mathrm{~Hz}, 2 \mathrm{H}, \mathrm{PymH}), 7.71$ $(\mathrm{t}, \mathrm{J}=5.9 \mathrm{~Hz}, 1 \mathrm{H}, \mathrm{PymH}), 7.33-7.20(\mathrm{~m}, 5 \mathrm{H}, \mathrm{PhH}), 6.56(\mathrm{t}, \mathrm{J}=4.5 \mathrm{~Hz}, 1 \mathrm{H}, \mathrm{NH}), 4.51(\mathrm{~d}, \mathrm{~J}=6.0$ $\left.\mathrm{Hz}, 2 \mathrm{H}, \mathrm{CH}_{2}\right) .{ }^{13} \mathrm{C}\left\{{ }^{1} \mathrm{H}\right\}$ NMR $\left(\delta, \mathrm{d}_{6}-\mathrm{DMSO}, 2^{\circ} \mathrm{C}\right): 164.1,159.7,142.2,129.9,128.7,128.2$, 
111.9, 64.7, 45.6. ESI-MS: $[\mathrm{M}+\mathrm{H}]^{+}$, found 186.03. $\left[\mathrm{C}_{11} \mathrm{H}_{12} \mathrm{~N}_{3}\right]^{+}$requires 186.10, MS-MS: 108.25, 91.39 .

\section{N-(4-((4-Toluidino)methyl)benzyl)-4-methylbenzenamine (32)}<smiles>Cc1ccc(CCc2ccc(CNCc3ccc(C)cc3)cc2)cc1</smiles>

colourless solid $246 \mathrm{mg}(78 \%) .{ }^{1} \mathrm{H}$ NMR $\left.\left(\delta, \mathrm{d}_{6}-\mathrm{DMSO}, 20^{\circ} \mathrm{C}\right)\right): 7.27(\mathrm{~s}, 4 \mathrm{H}, \mathrm{PhH}), 6.84(\mathrm{~d}, \mathrm{~J}=$ $7.0 \mathrm{~Hz}, 4 \mathrm{H}, \mathrm{PhH}), 6.49$ (d, J=6.5 Hz, 4H, PhH), $5.94(\mathrm{~s}, 2 \mathrm{H}, \mathrm{NH}), 4.18\left(\mathrm{~s}, 4 \mathrm{H}, \mathrm{CH}_{2}\right), 2.12(\mathrm{~s}$, $\left.6 \mathrm{H}, \mathrm{CH}_{3}\right) .{ }^{13} \mathrm{C}\left\{{ }^{1} \mathrm{H}\right\}$ NMR $\left(\delta, \mathrm{d}_{6}-\mathrm{DMSO}, 20^{\circ} \mathrm{C}\right): 149.9,142.2,132.7,130.6,127.5,115.9,50.0$, 23.6. ESI-MS: $[\mathrm{M}+\mathrm{H}]^{+}$, found 317.03. $\left[\mathrm{C}_{22} \mathrm{H}_{25} \mathrm{~N}_{2}\right]^{+}$requires 317.2018, HRMS (ESI): $[\mathrm{M}+\mathrm{H}]^{+}$, found 317.2015 .

\section{References}

1 Perrin, D. D.; Armarego, W. L. F. Purification of Laboratory Chemicals, 3rd ed.; Pergamon: New York, 1988.

2 Murugesan, S.; Stöger, B.; Carvalho, M. D.; Ferreira, L. P.; Pittenauer, E.; Allmaier, G.; Veiros, L. F.; Kirchner, K. Organometallics 2014, 33, 6132.

3 Sur, S. K. J. Magn. Reson. 1989, 82, 169.

4 (a) Carlin, R. L. Magnetochemistry; Springer-Verlag: Heidelberg, 1986. (b) Orchard, A. F. Magnetochemistry; Oxford University Press, 2003.

$5 \quad$ Wilson, F. G.; Wheeler, T. S. Org. Synth. 1928, 8, 38.

6 Nystrom, R. F.; Brown, W. G. J. Am. Chem. Soc. 1948, 70, 3738-40.

7 Billman, J. H.; Radike, A.; M., B. W. J. Am. Chem.Soc. 1942, 64, 2977-2978.

8 Rice, R. G.; Kohn, E. J. Org. Synth. 1956, 36, 21-23.

9 Alper, H.; Tanaka, M. Synthesis 1978, 10, 781-782.

10 Shawcross, A. P.; Stanforth, S. P. Tetrahedron 1989, 45, 7063-7076.

11 Zhang, Z.; Gao, J.; Wang, G.-W. Org. \& Biomol. Chem. 2005, 3, 1617-1619.

12 Mangat, R.; Baljit, K.; Dhir, B. S. J. Indian Chem. Soc. 1982, 59, 416-417.

13 Castro, L. C.; Misal Sortais, J.-B..; Darcel, C. Chem. Comm. 2012, 48, 151-153.

14 Zhiyan, X.; Fei, Y.; Zongru, G.; Jinying, T.; Junzheng, L.; Shuen, Z.; Feilin, N.; Rongya, T.; Xiaolin, Z.; Junyang, L. 2011, CN 102030700.

15 Juaristi, E.; Reyna, J. D. Tetrahedron Lett. 1984, 25, 3521-3524.

16 Diez, V.; Cuevas, J. V.; Garcia-Herbosa, G.; Aullon, G.; Charmant, J. P. H.; Carbayo, A.; Munoz, A. Inorg. Chem. 2007, 46, 568-577.

17 Ryashentseva, M. A.; Minachev, K. M.; Kalinovskii, O. A.; Gol'dfarb, Ya. L. Zhurnal Organicheskoi Khimii 1965, 1, 1104-1108. 
18 Zhou, W.; Fan, Mengyang; Yin, J.; Jiang, Y.; Ma, D. J. Am. Chem. Soc. 2015, 137, 1194211945.

19 Moreno-Manas, M.; Morral, L.; Pleixats, R. J. Org. Chem. 1998, 63, 6160-6166.

20 Kaloglu, N.; Ozdemir, I.; Gurbuz, N.; Achard, M.; Bruneau, C. Cat. Comm. 2016, 74, 33-38.

21 Pratt, E. F.; Frazza, E. J. J. Am. Chem. Soc. 1954, 76, 6174-6179.

22 Singh, S.; Sharma, V. K. Tetrahedron Lett. 1979, 29, 2733-2734.

23 Zhang, Z.; Mao, J.; Zhu, D.; Wu, F.; Chen, H.; Wan, B. Tetrahedron 2006, 62, 4435-4443.

24 Trifonov, L.; Orakhovats, A. Helv. Chim. Acta 1987, 70, 1732-1736.

25 Li, F.; Chen, L.; Kang, Q.; Cai, J.; Zhu, G. New J. Chem. 2013, 37, 624-631.

26 Fioravanti, R.; Biava, M.; Porretta, G. C.; Landolfi, C.; Simonetti, N.; Villa, A.; Conte, E.; PortaPuglia, A. Eur. J. Med. Chem. 1995, 30, 123-132.

6. ${ }^{1} \mathrm{H}$ and ${ }^{13} \mathrm{C}\left\{{ }^{1} \mathrm{H}\right\}$ NMR Spectra of Organic Products 


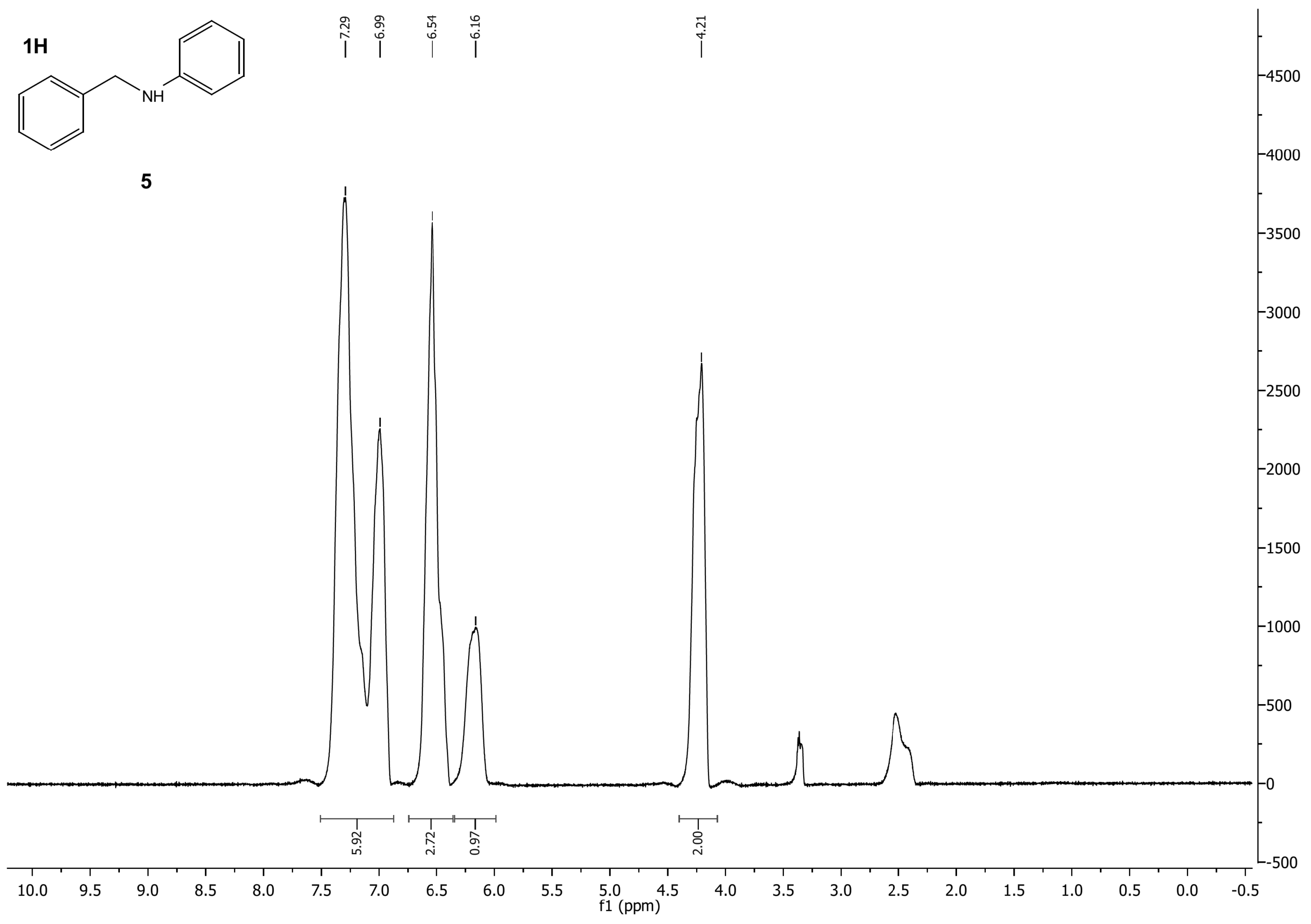




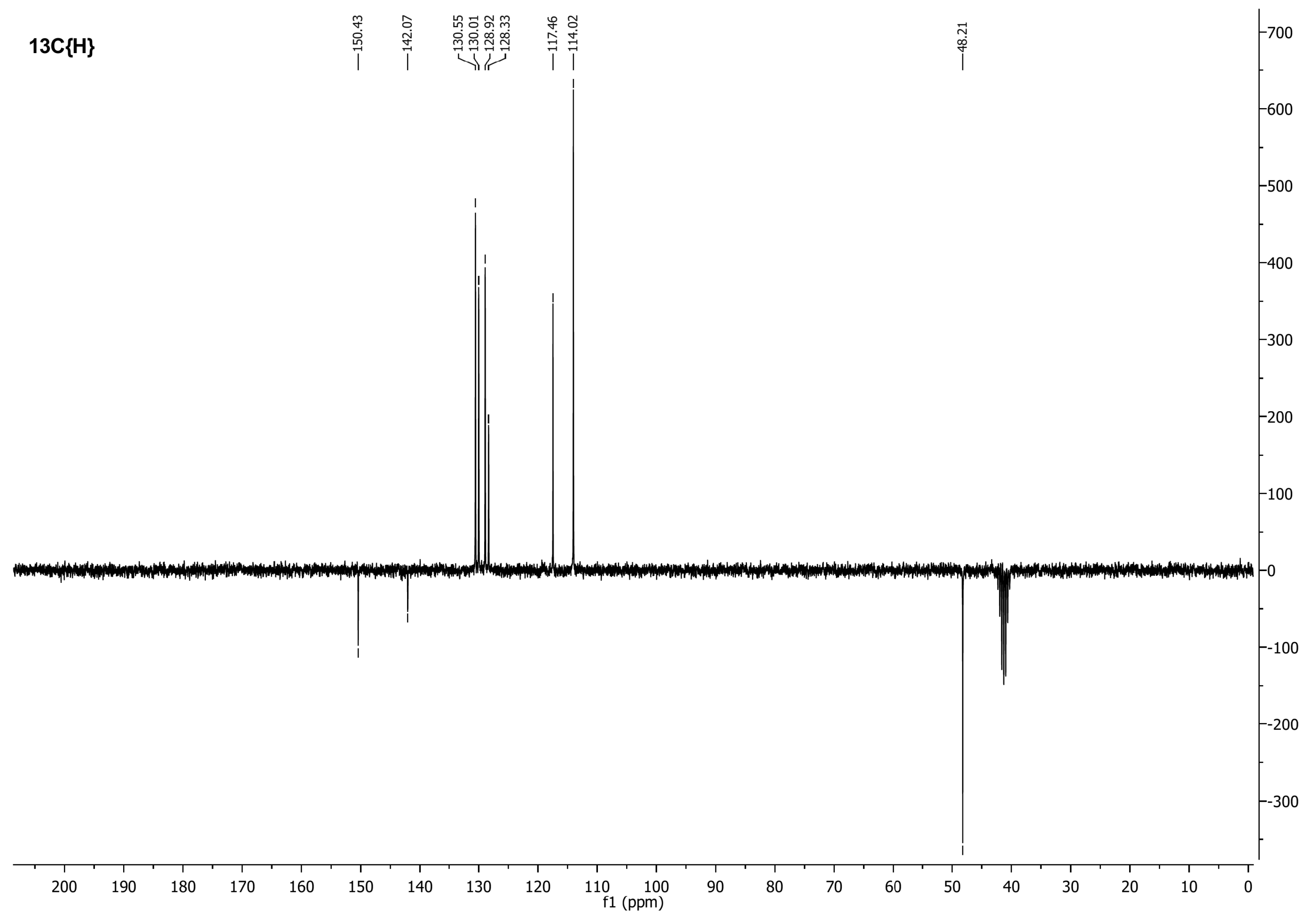




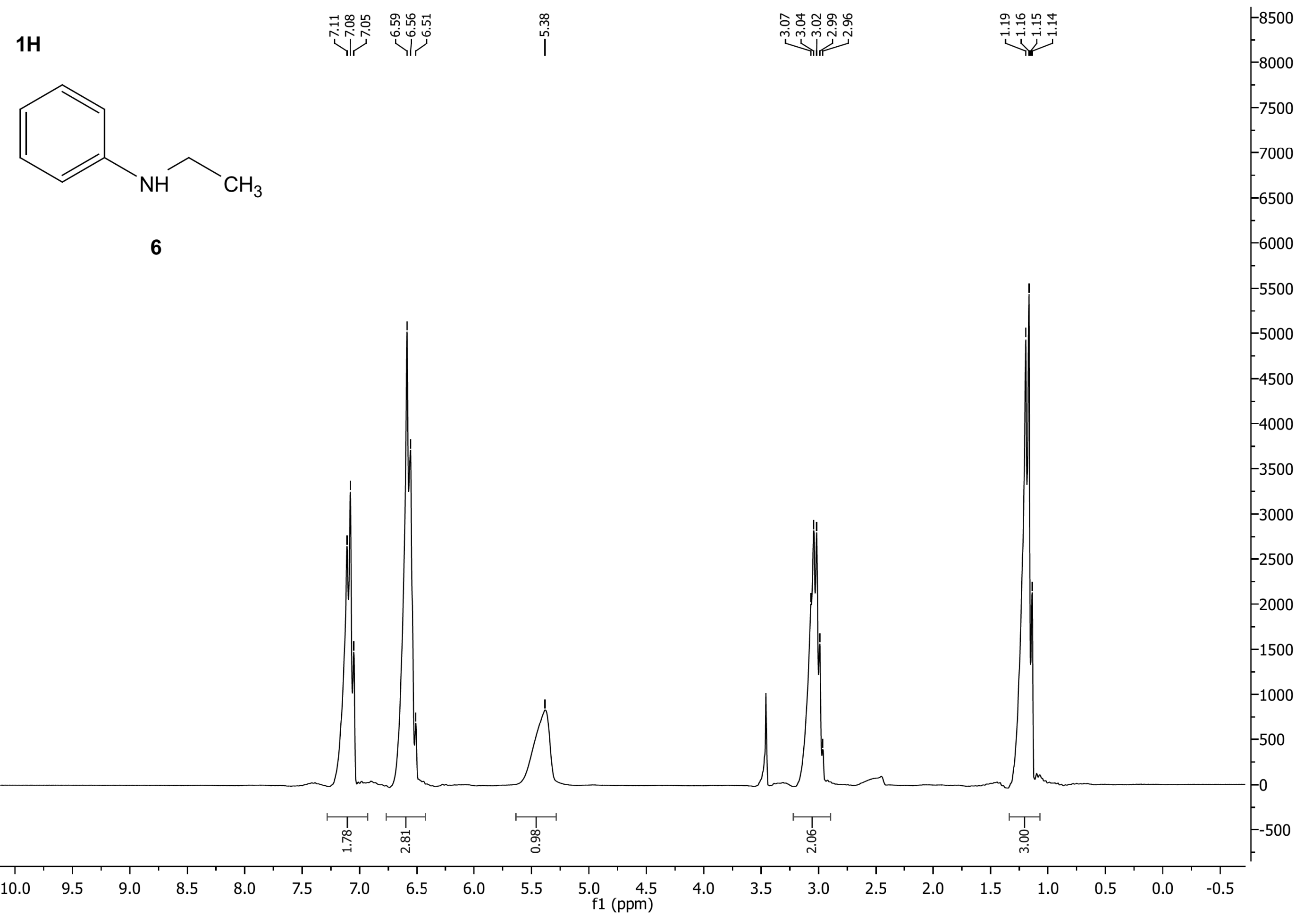




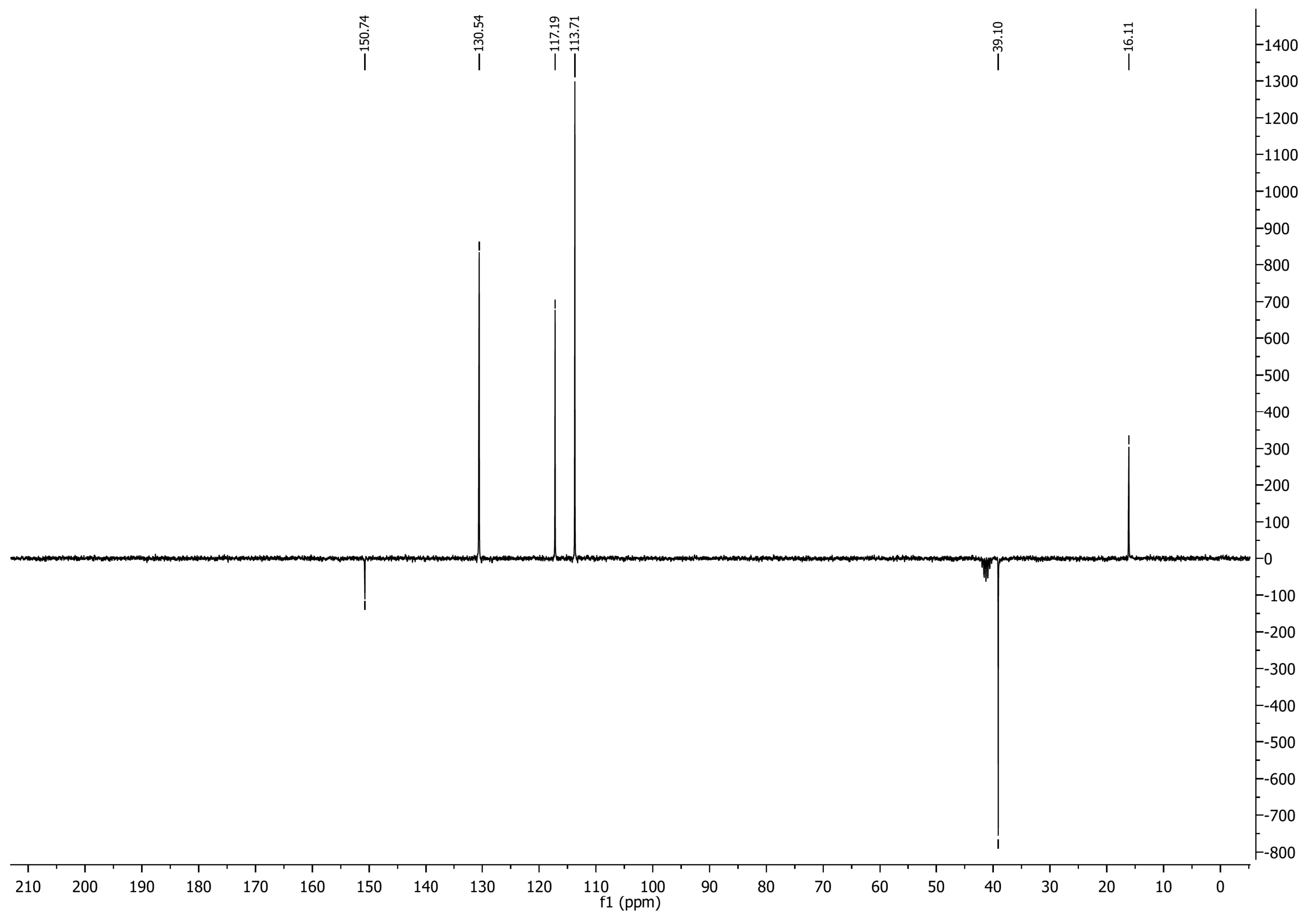




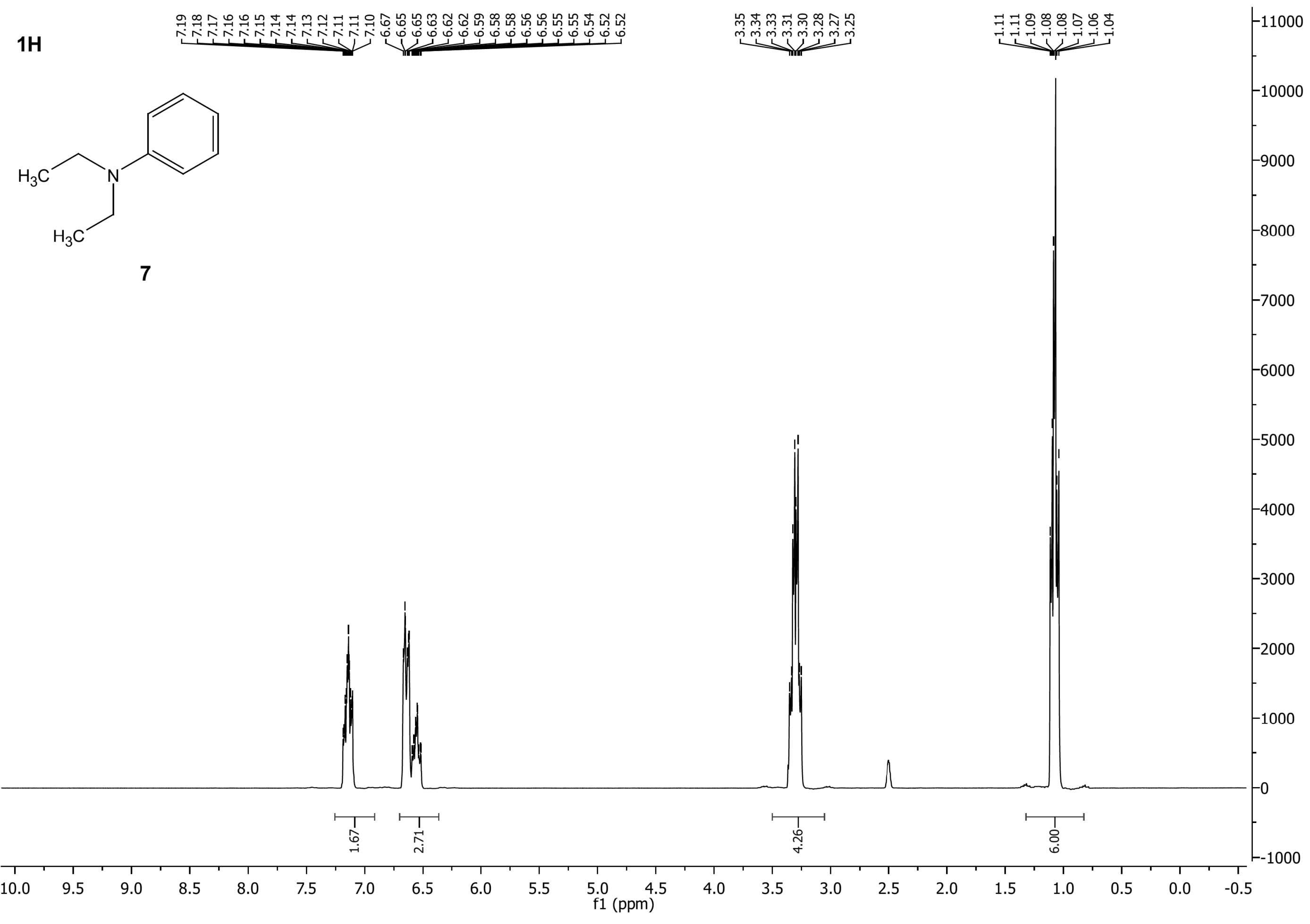




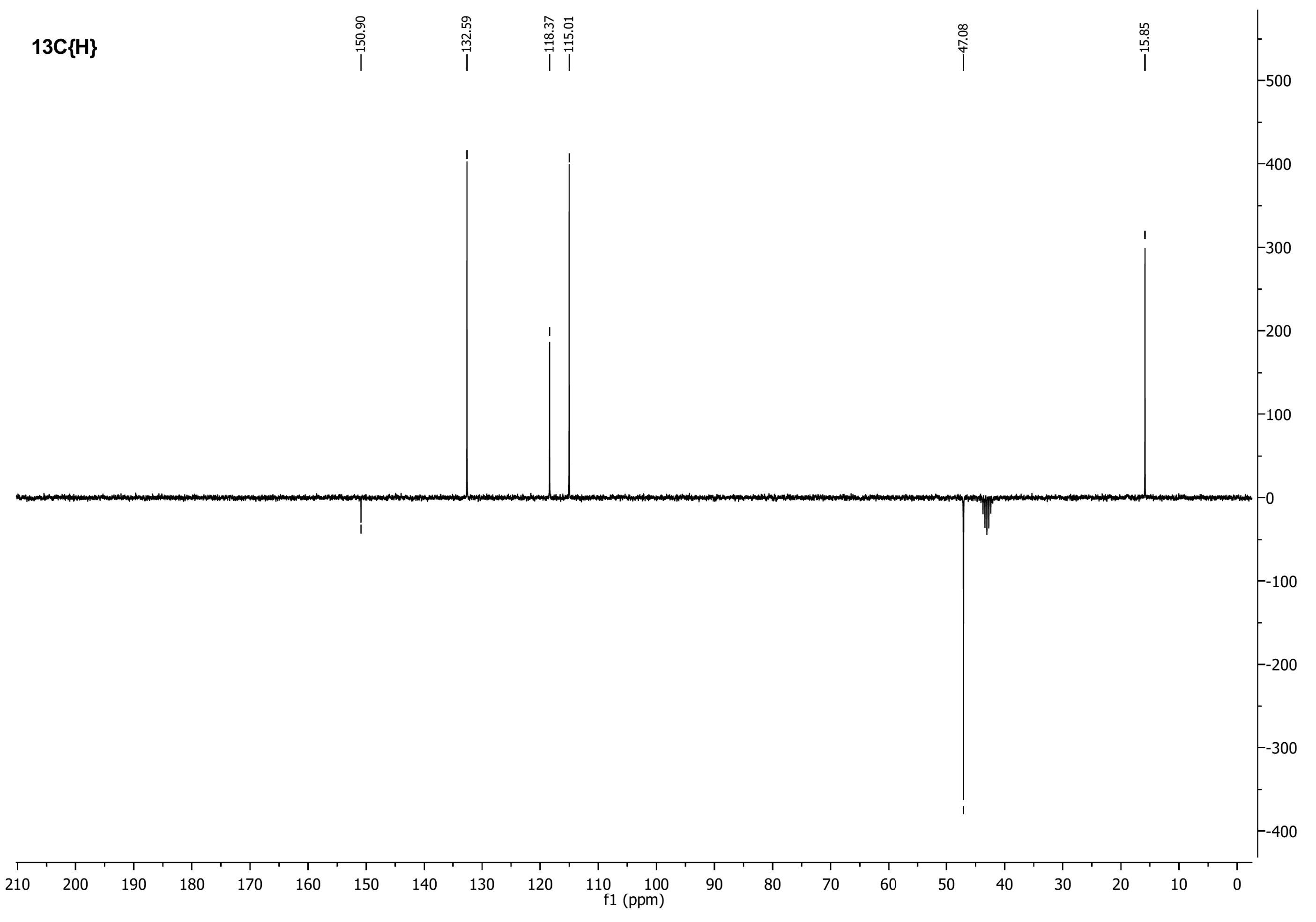




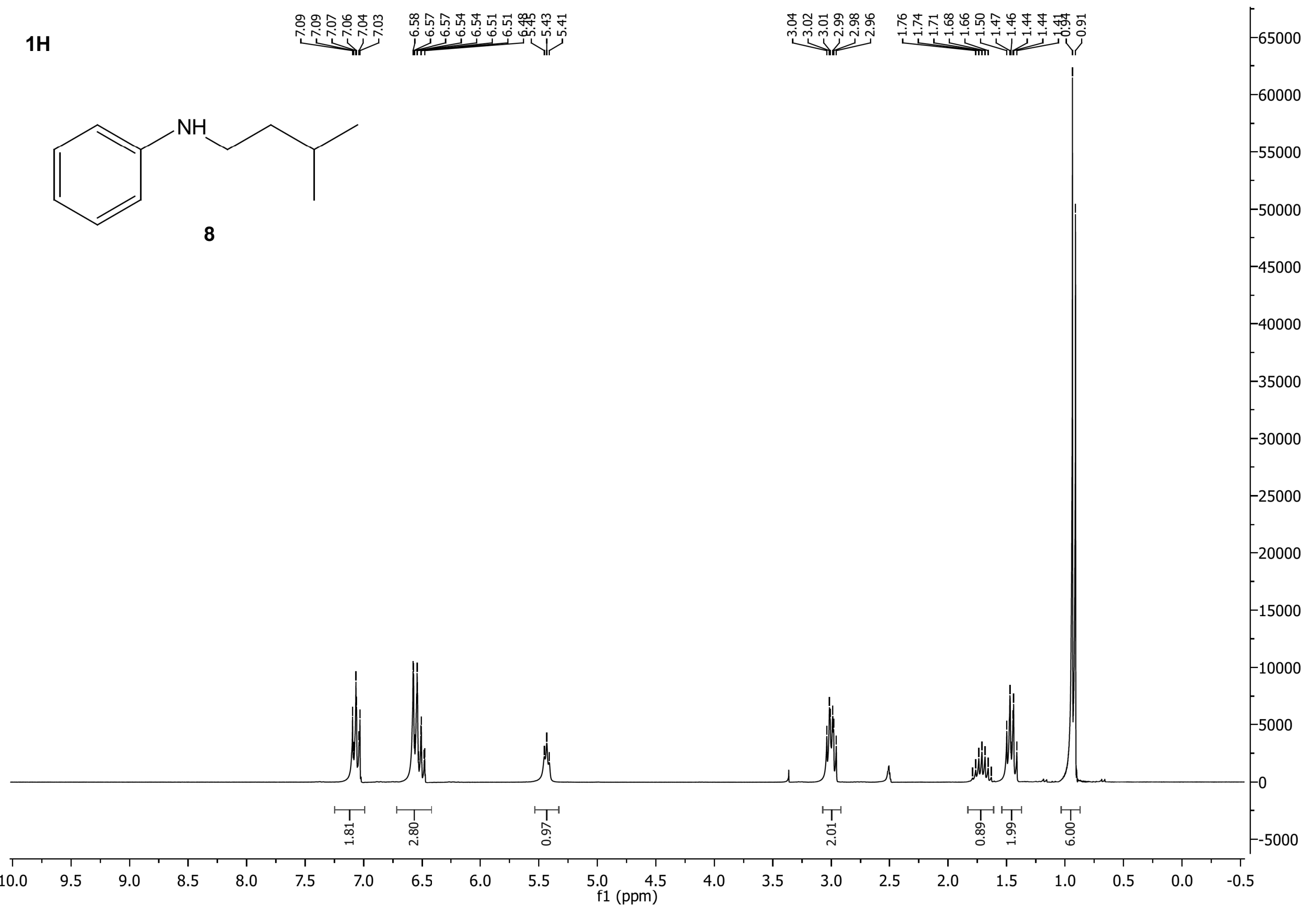




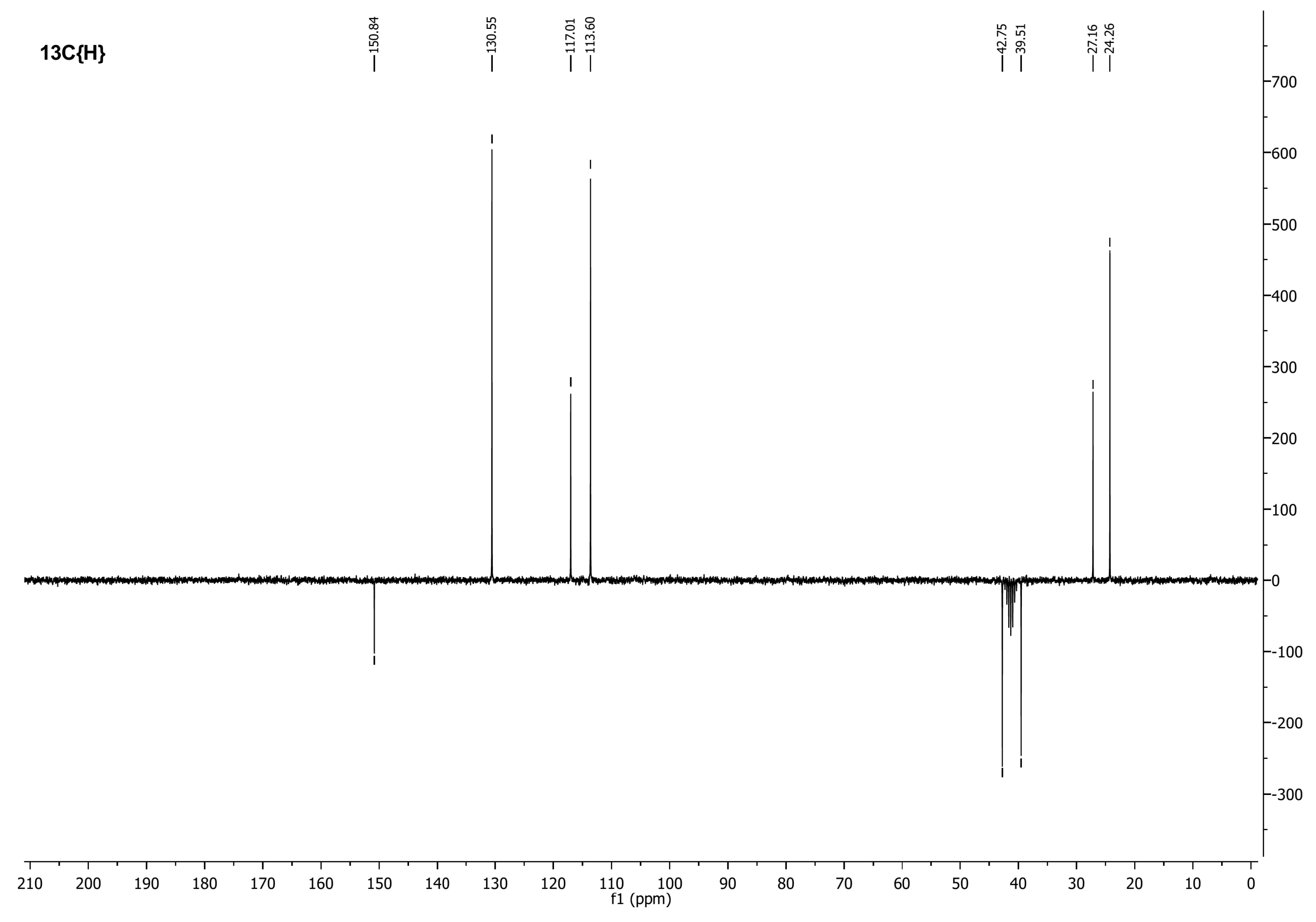




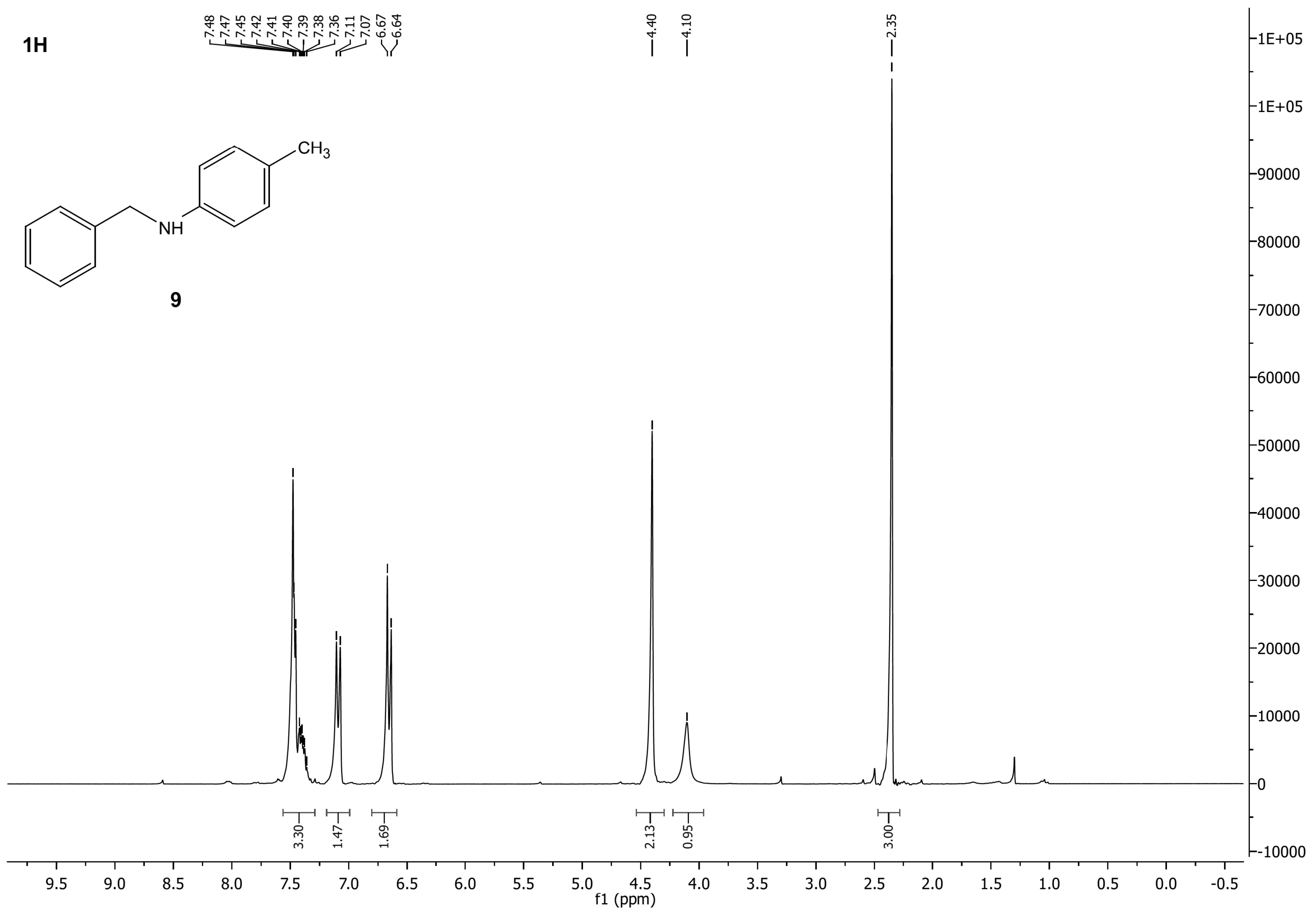




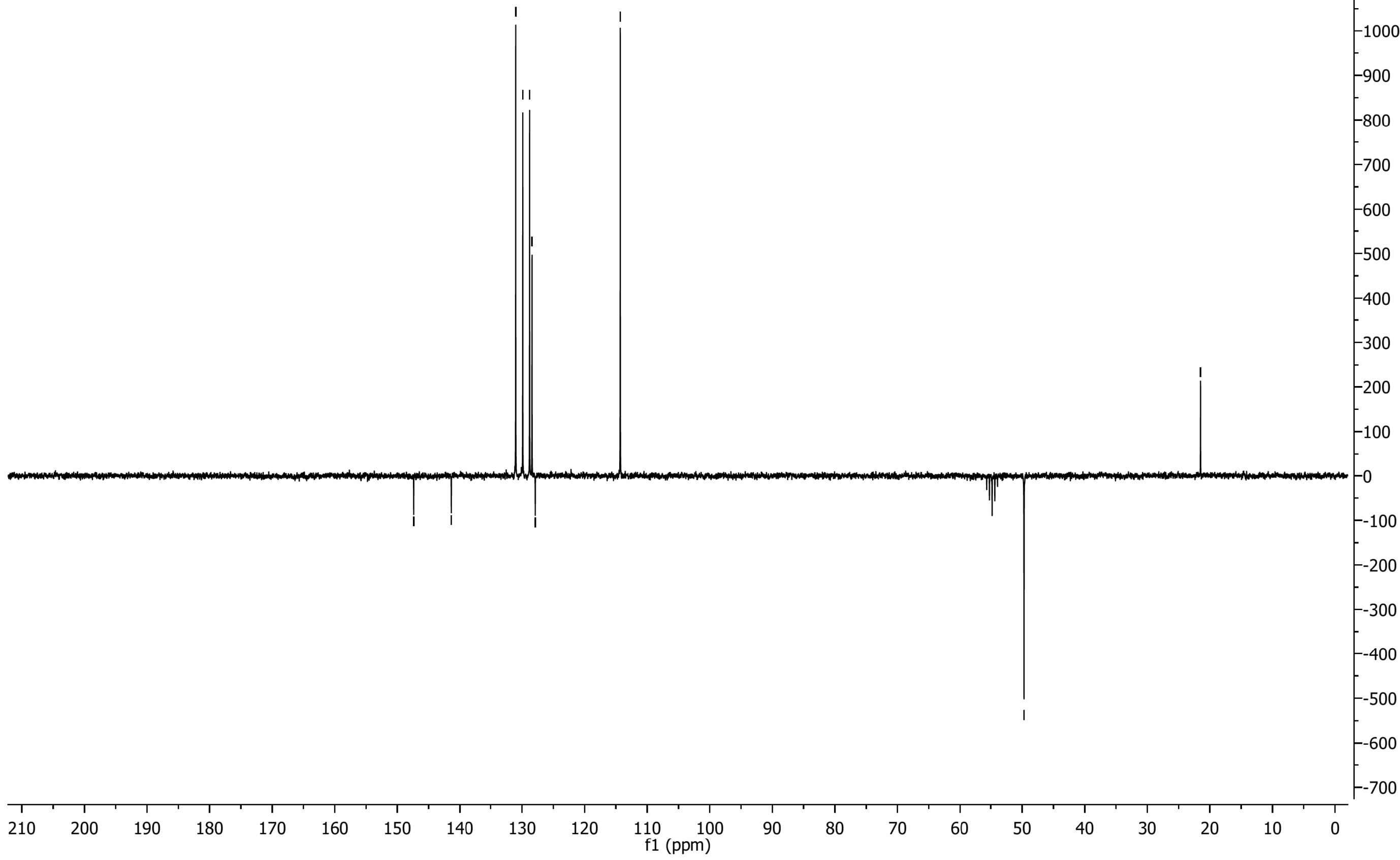




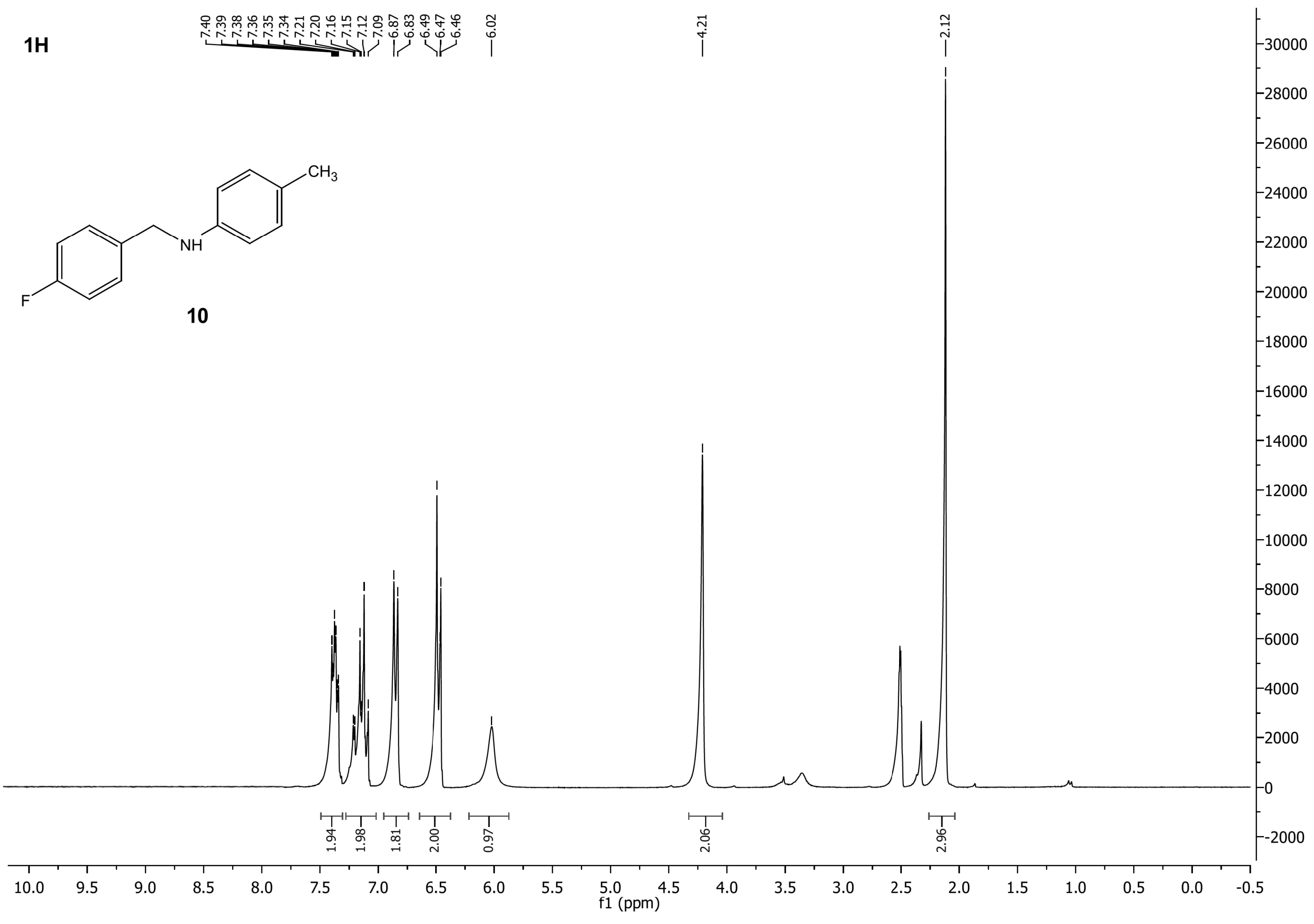




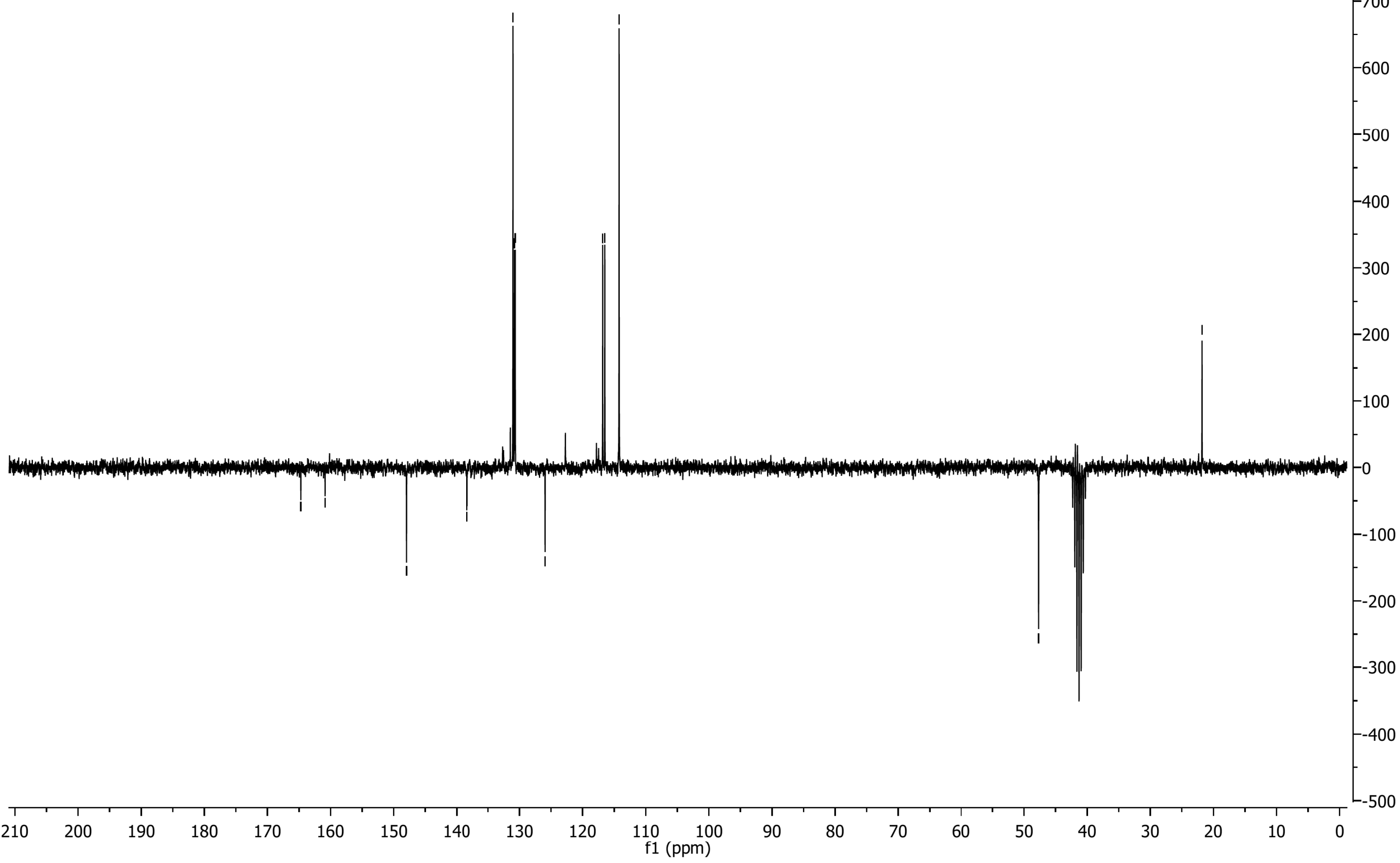




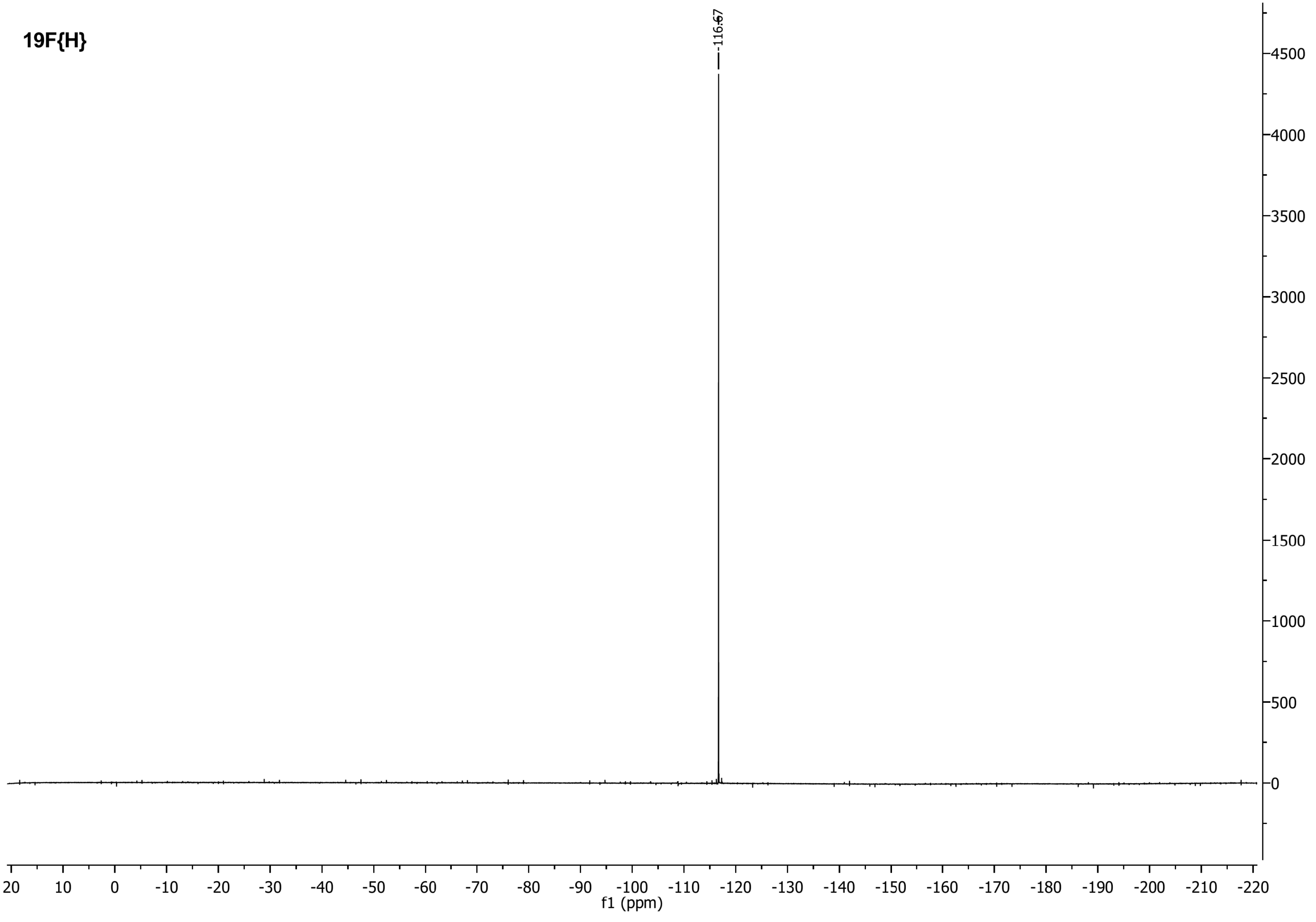




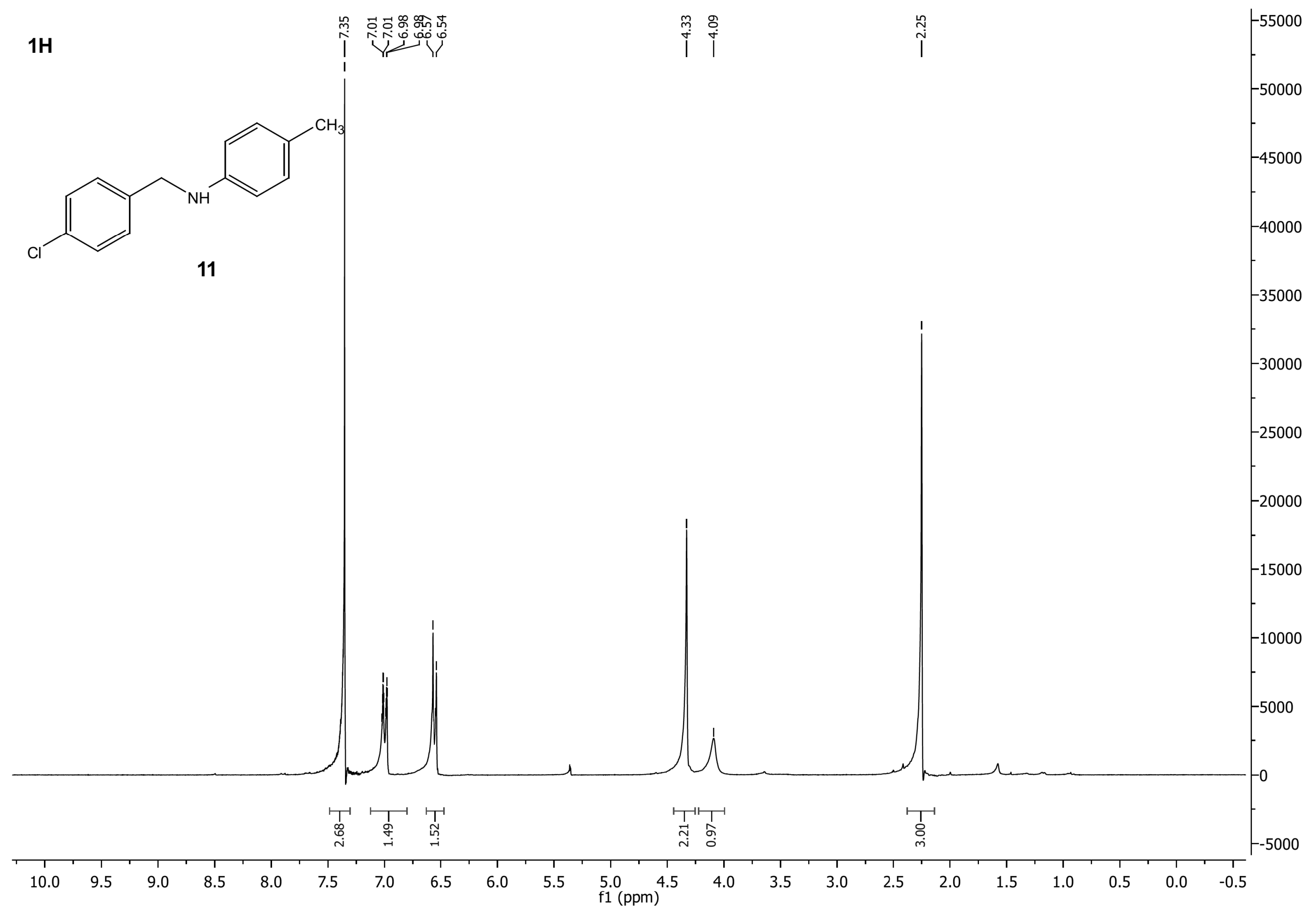




\section{$13 \mathrm{C}\{\mathrm{H}\}$}

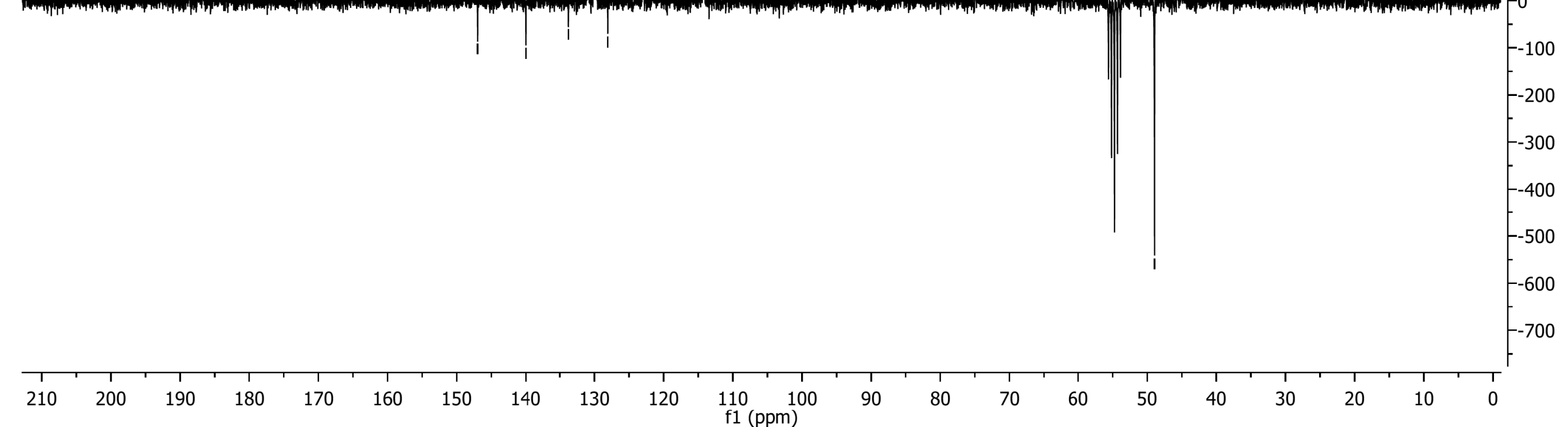


<smiles>COc1ccc(CNc2ccc(C)cc2)cc1OC</smiles>

12
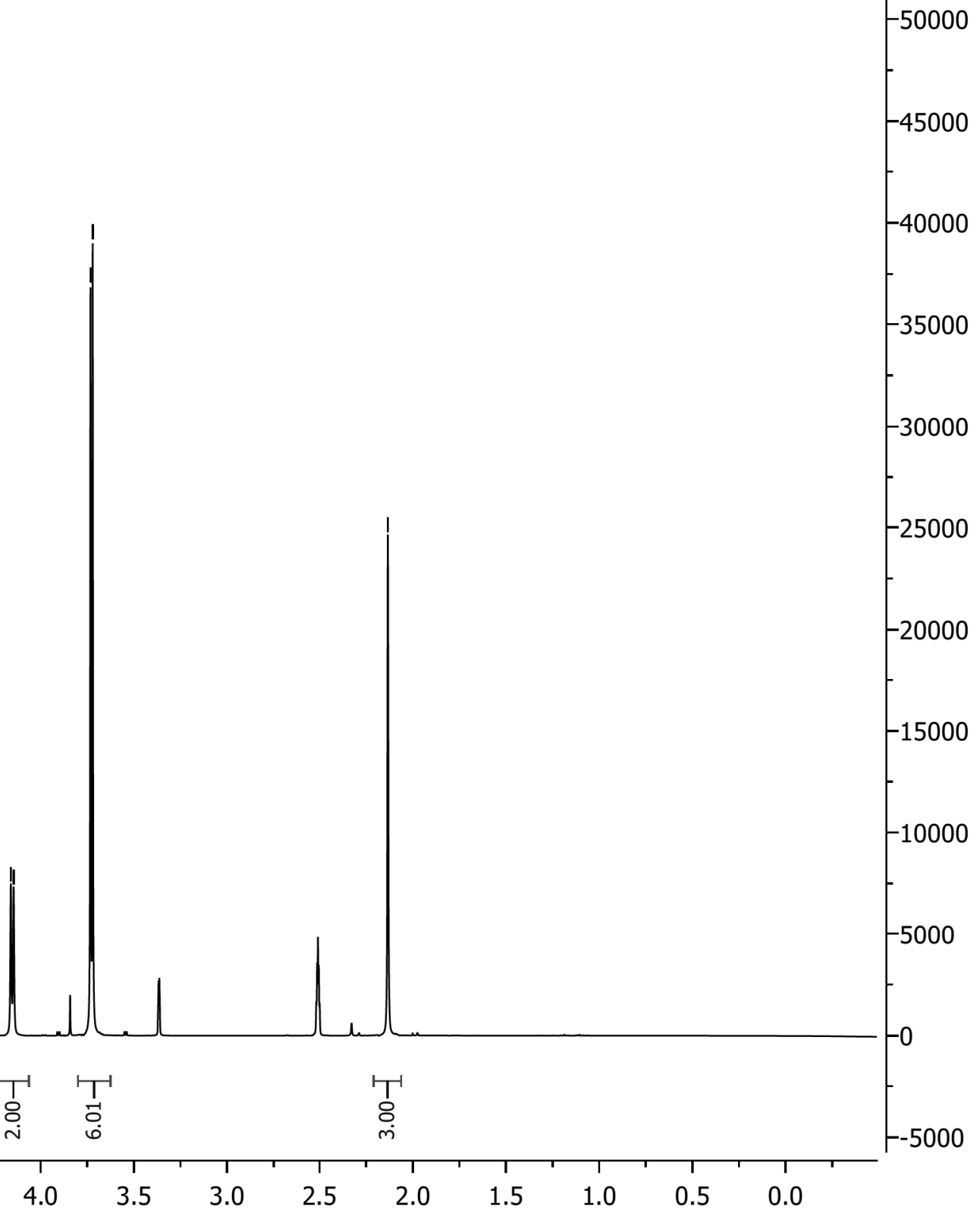

35000
30000
25000
20000
15000
10000
5000
-5000




\section{$13 C\{H\}$}

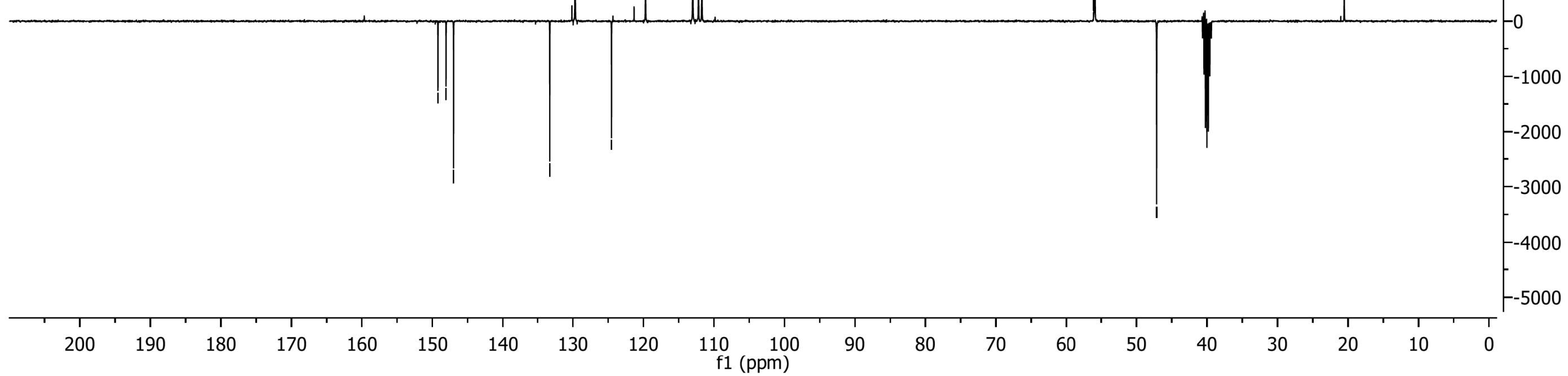




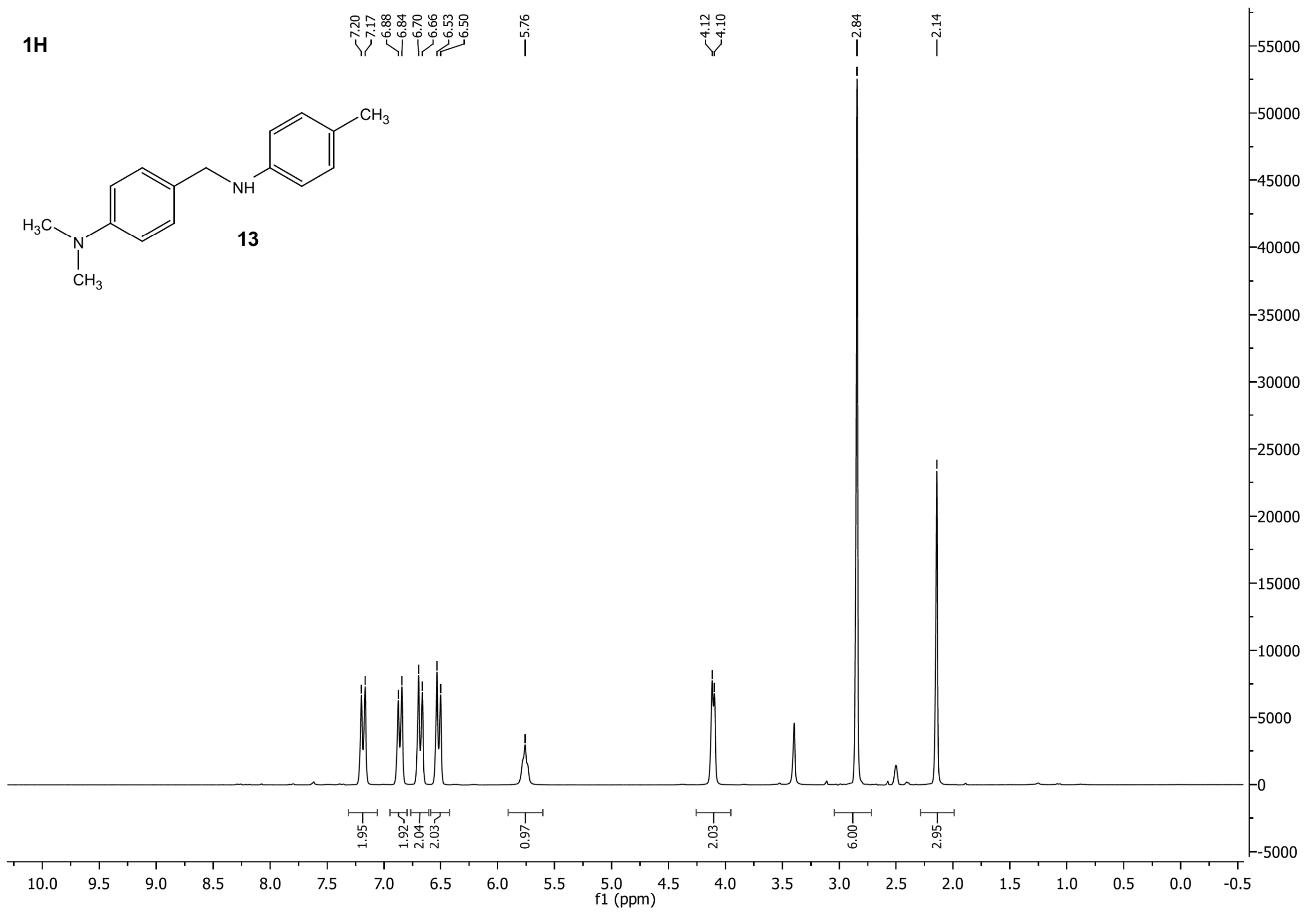




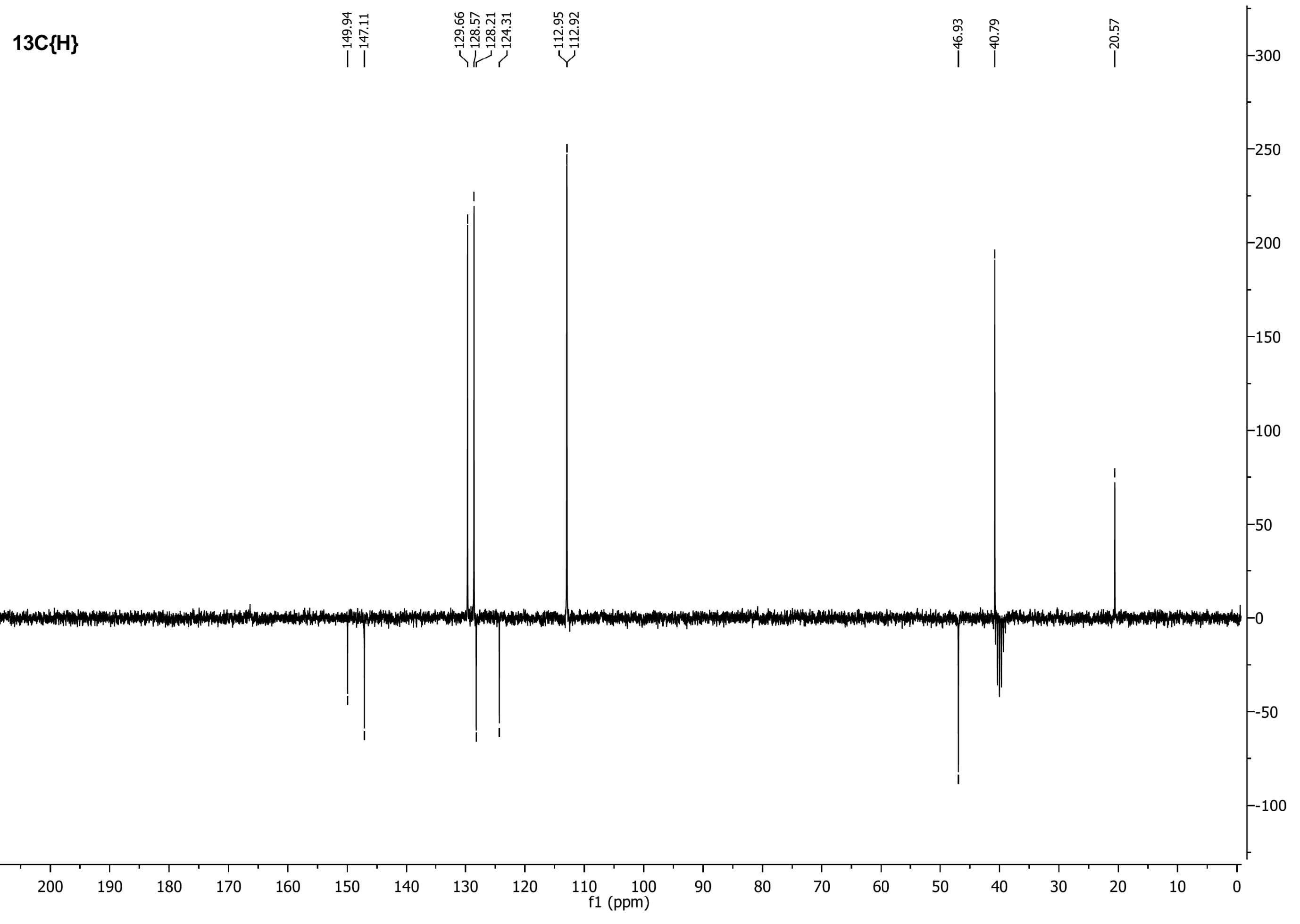




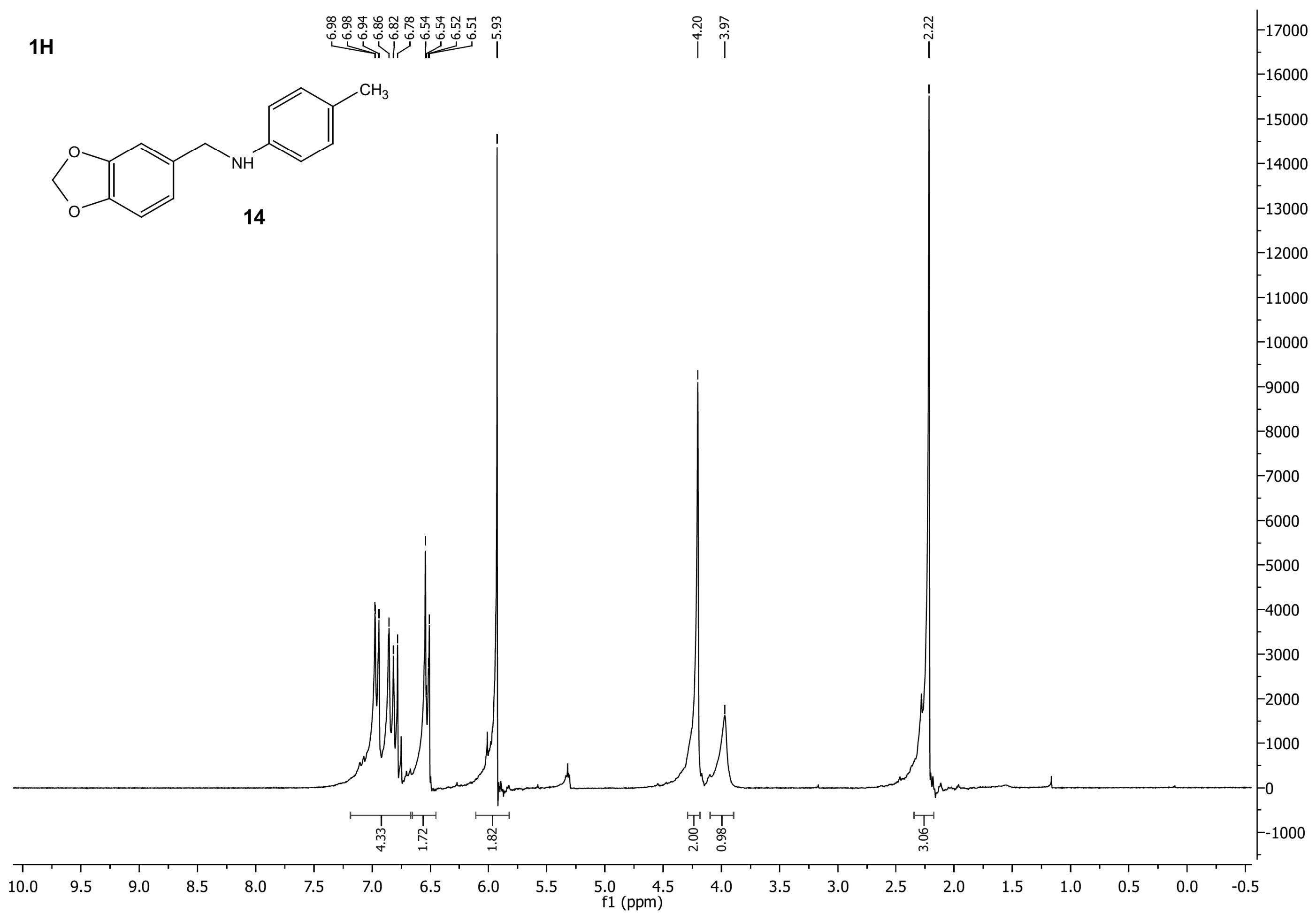




\section{$13 C\{H\}$}

रा

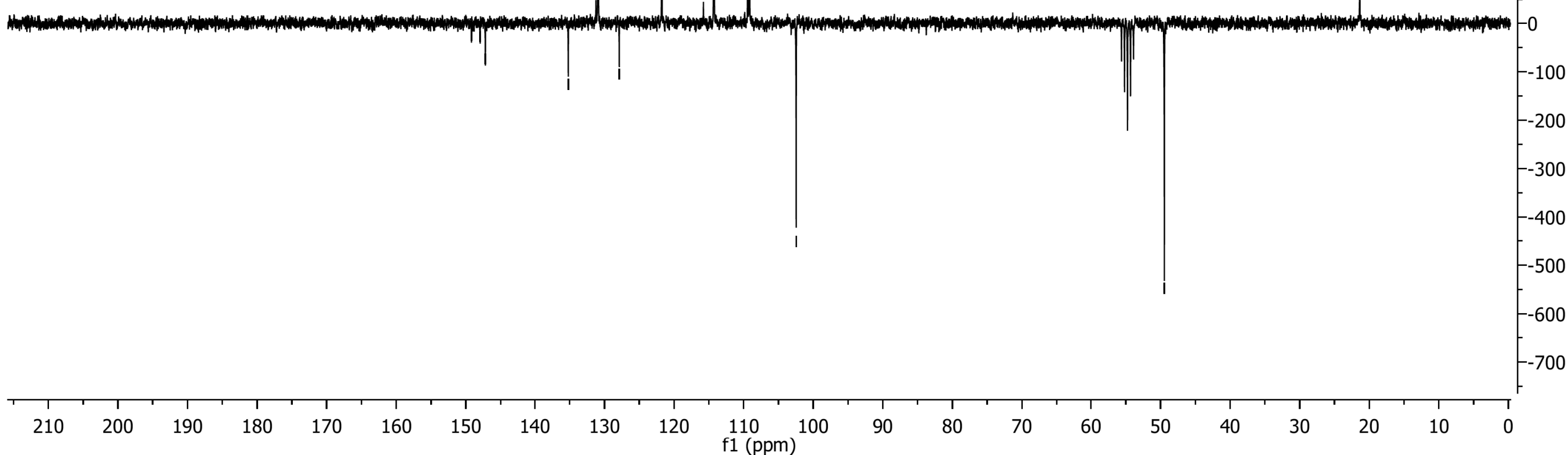




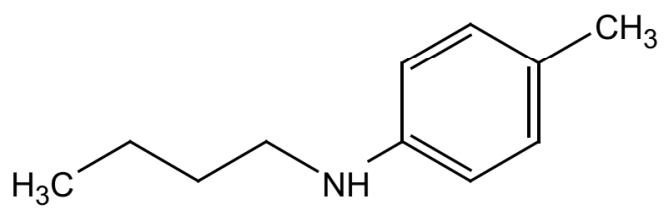

15

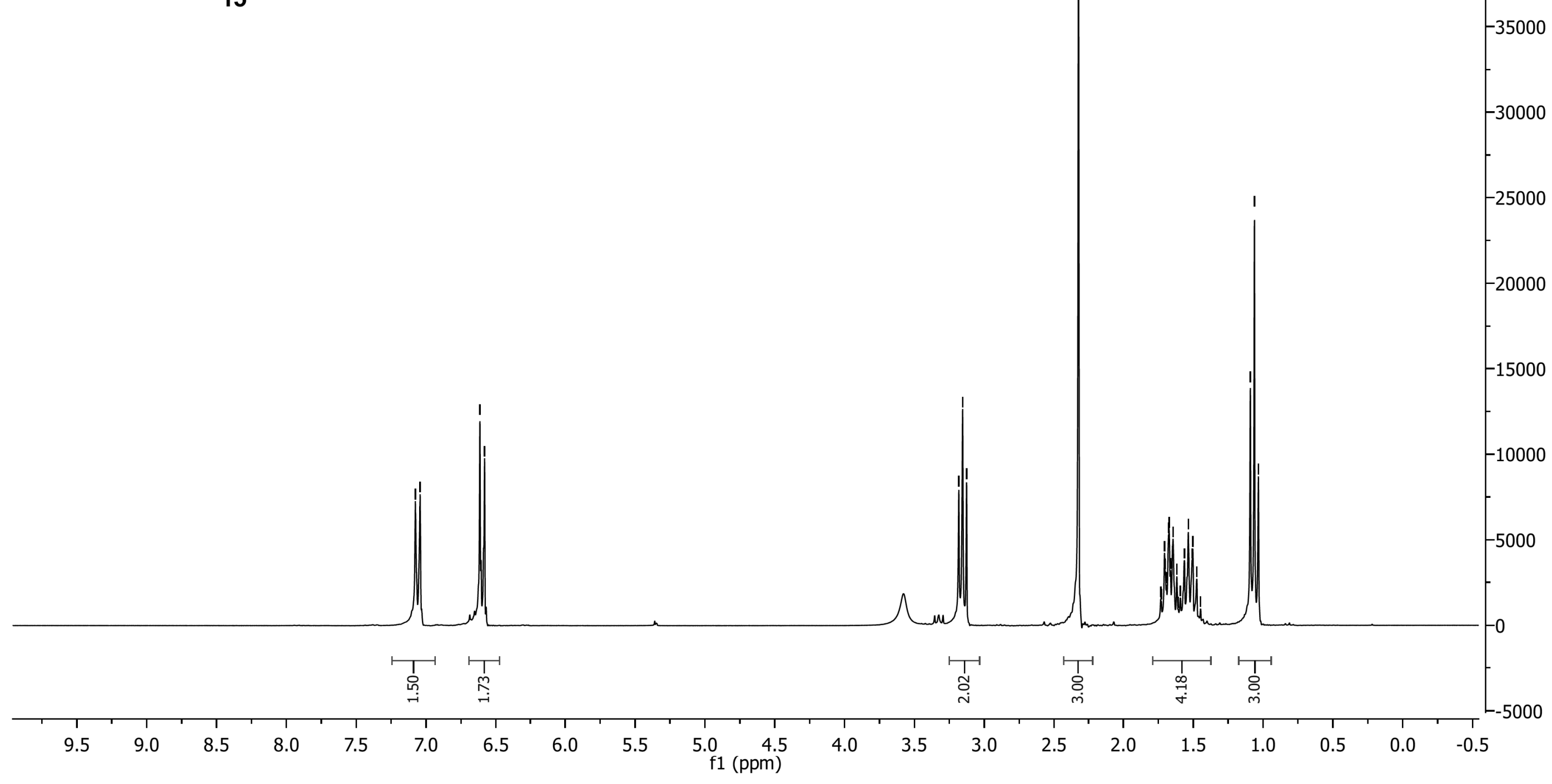




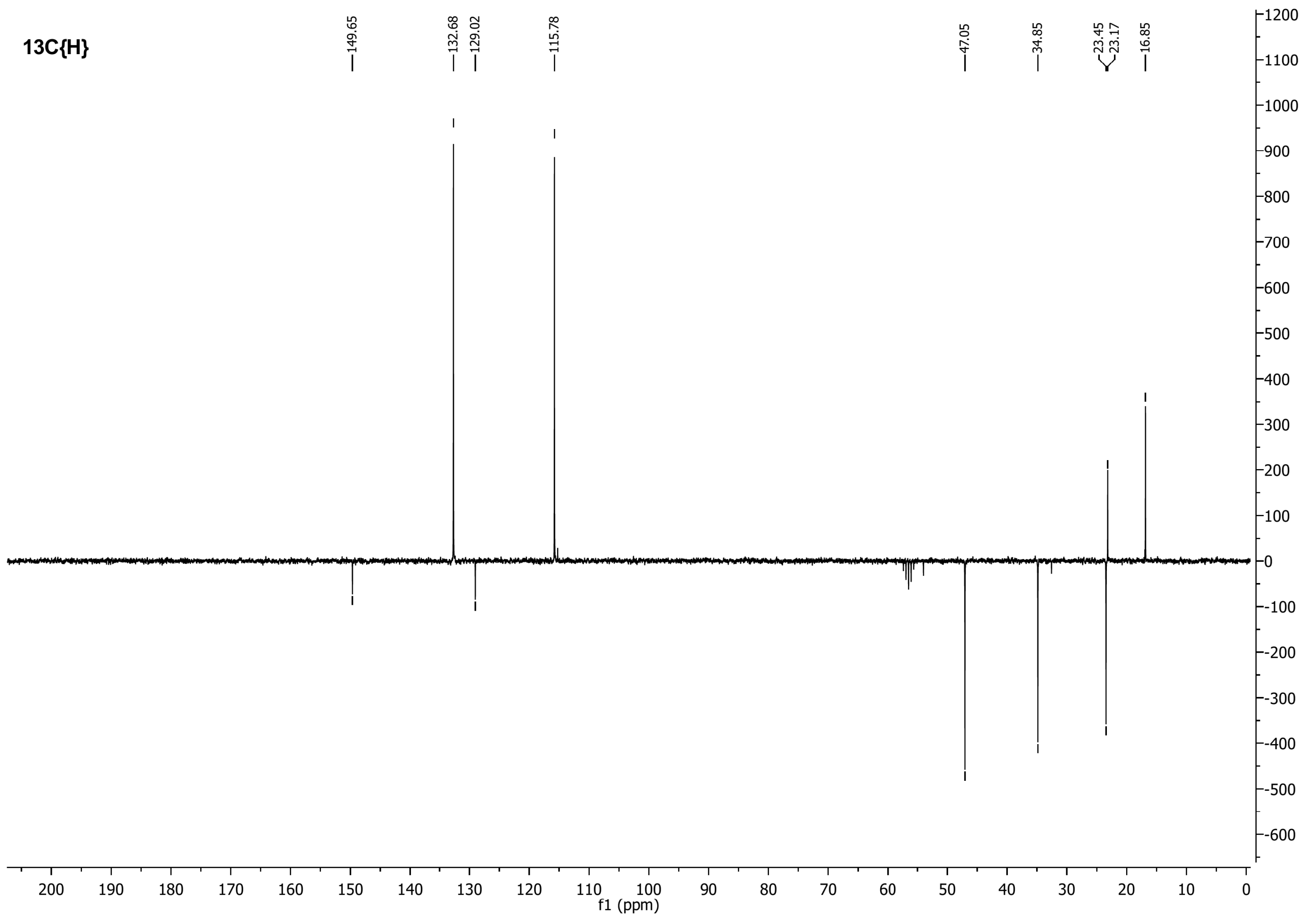




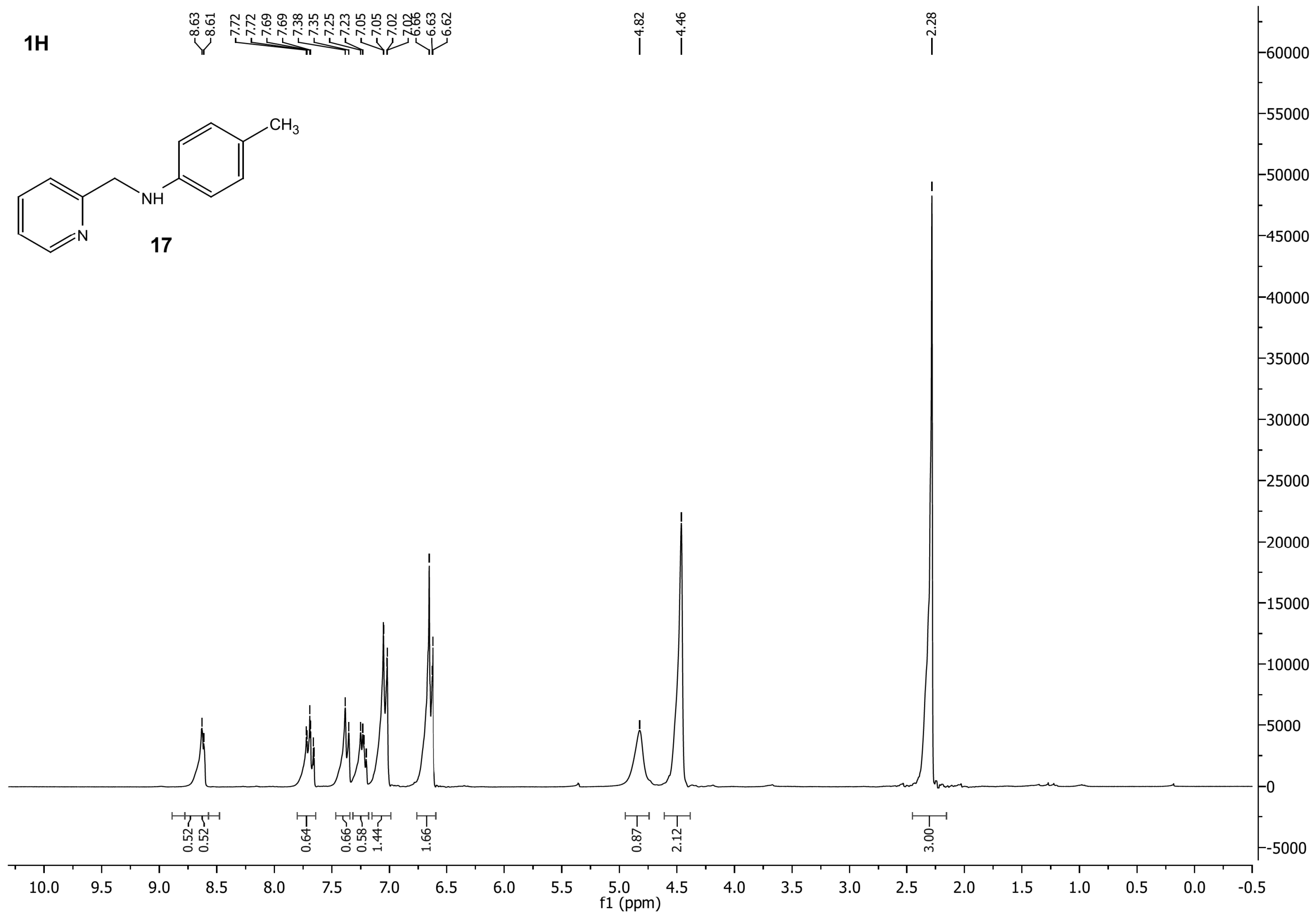




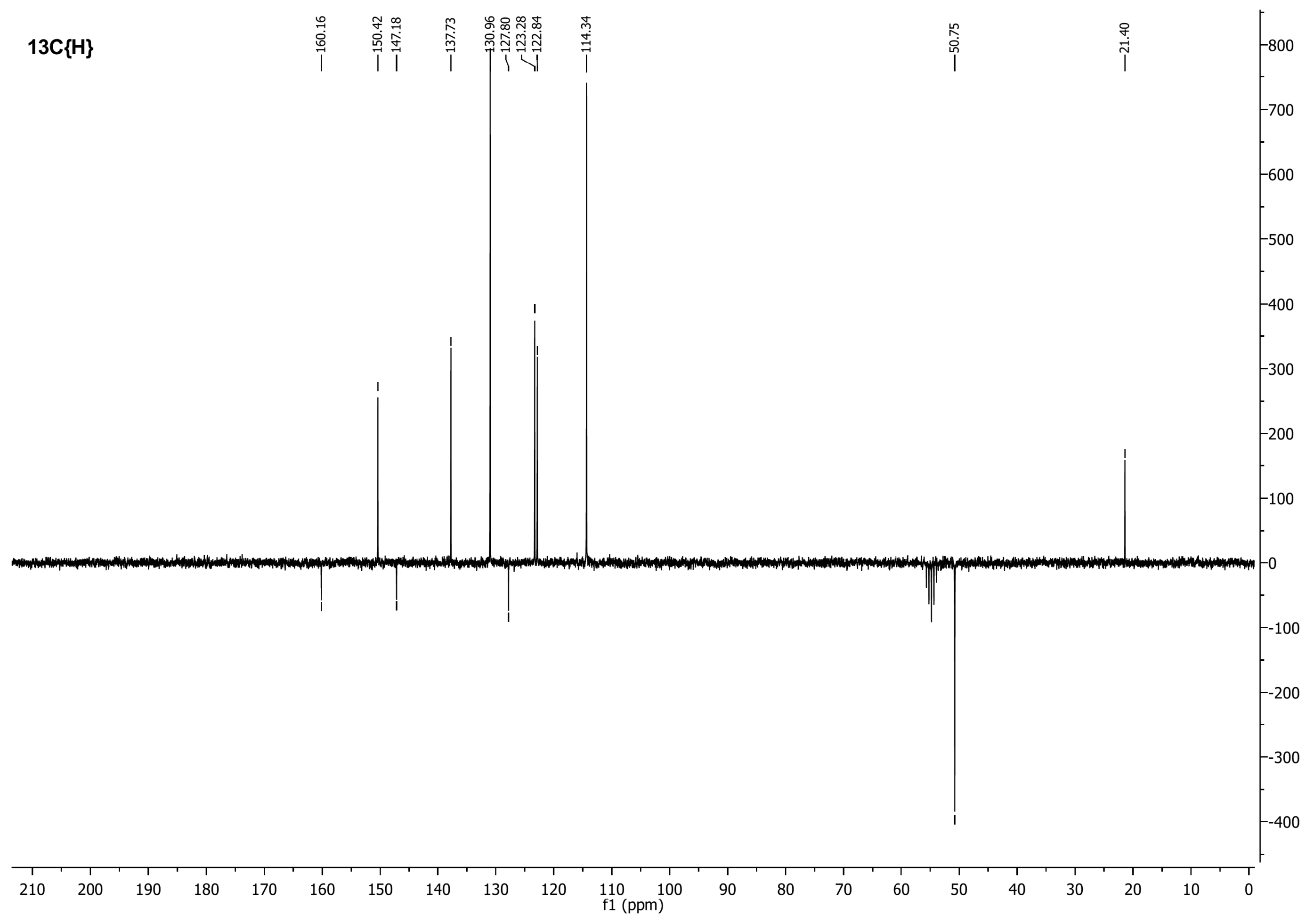




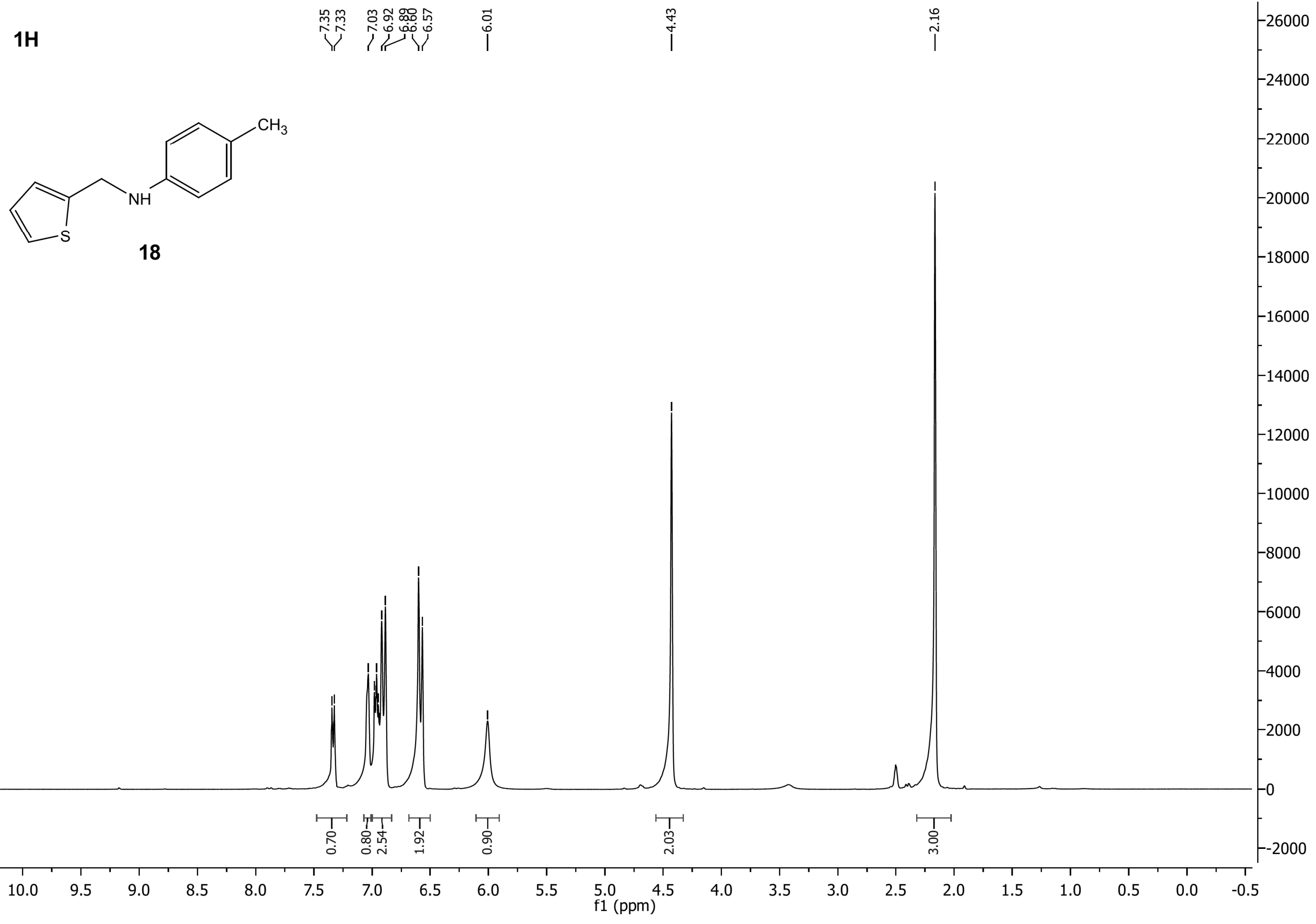




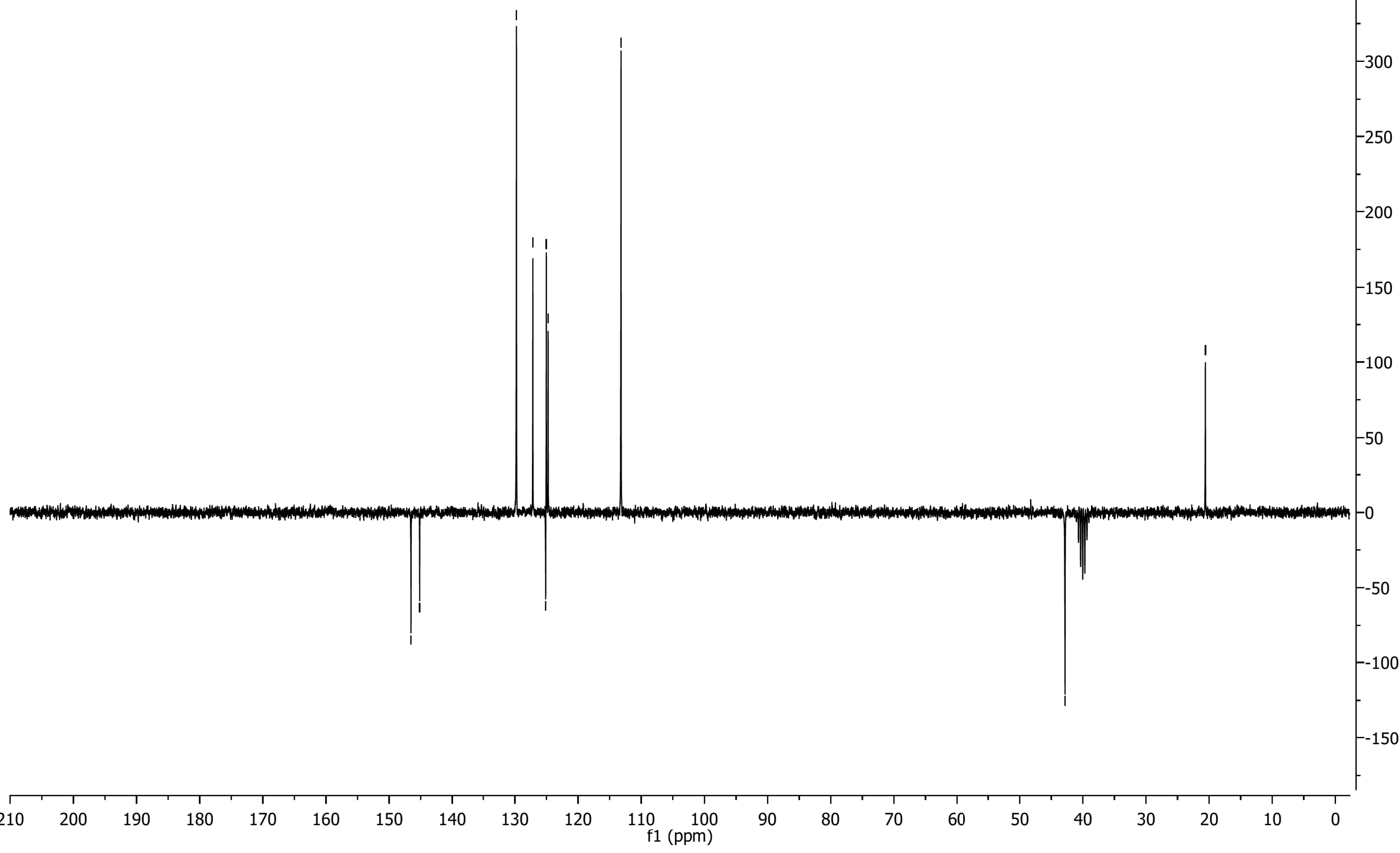




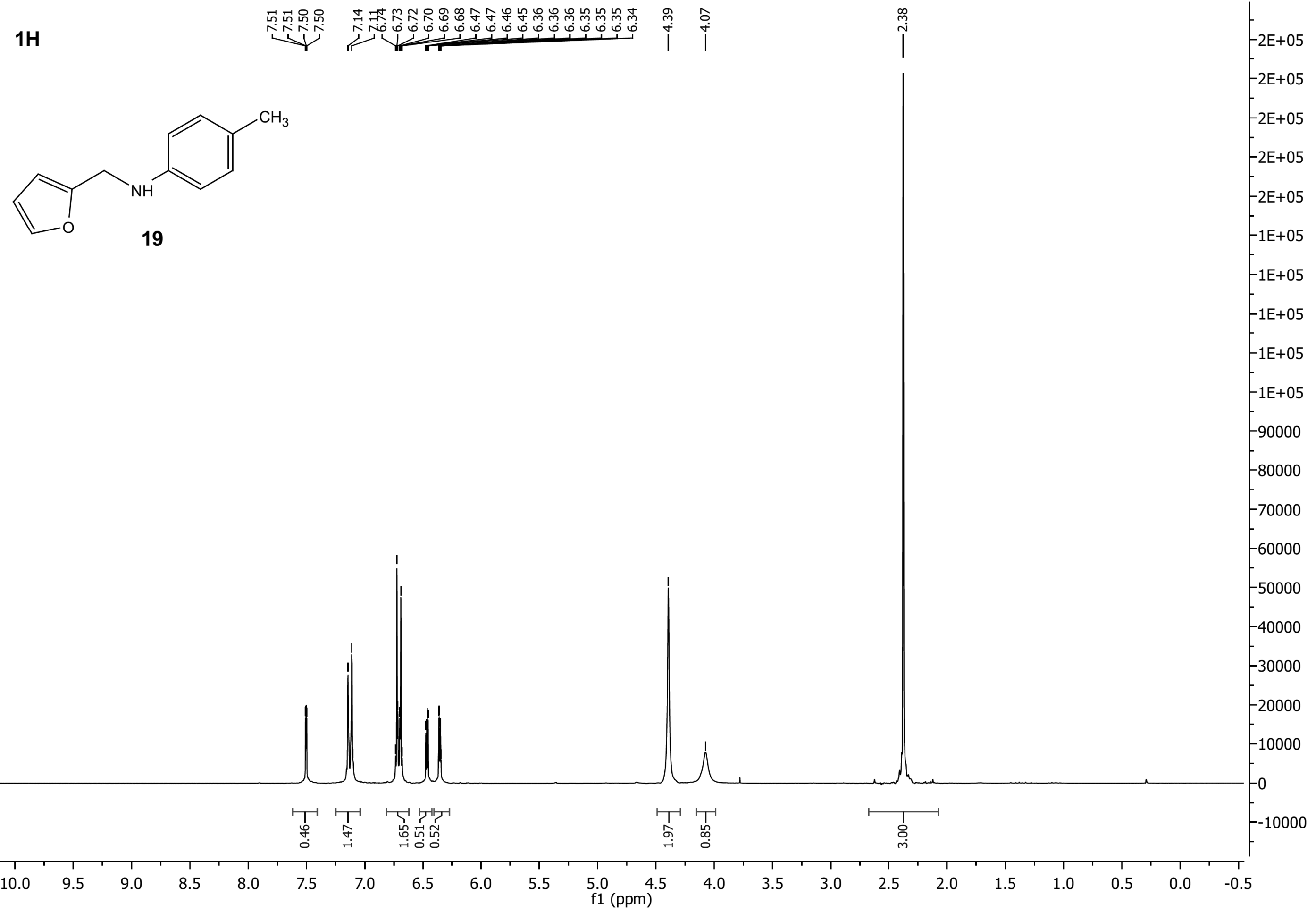




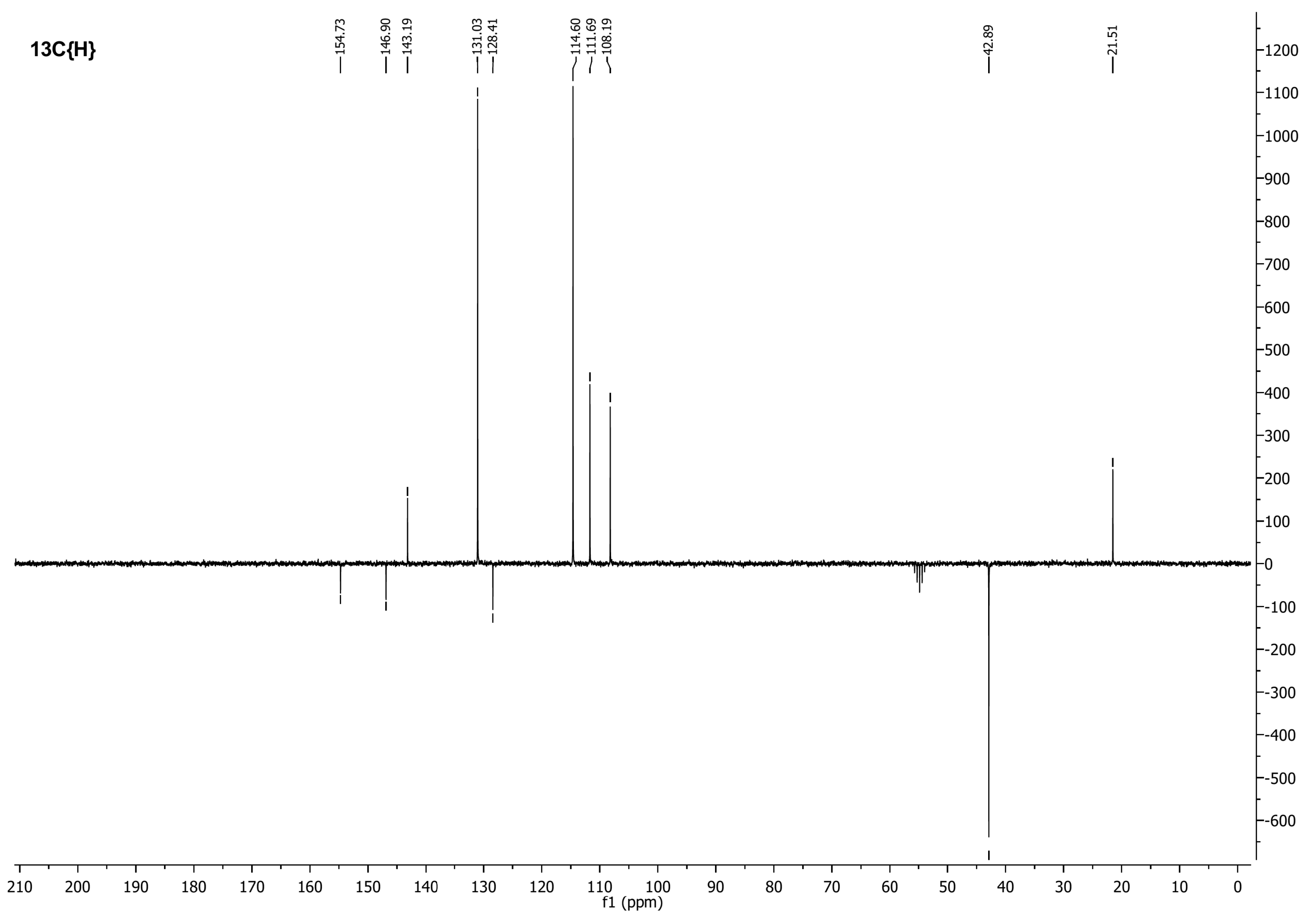




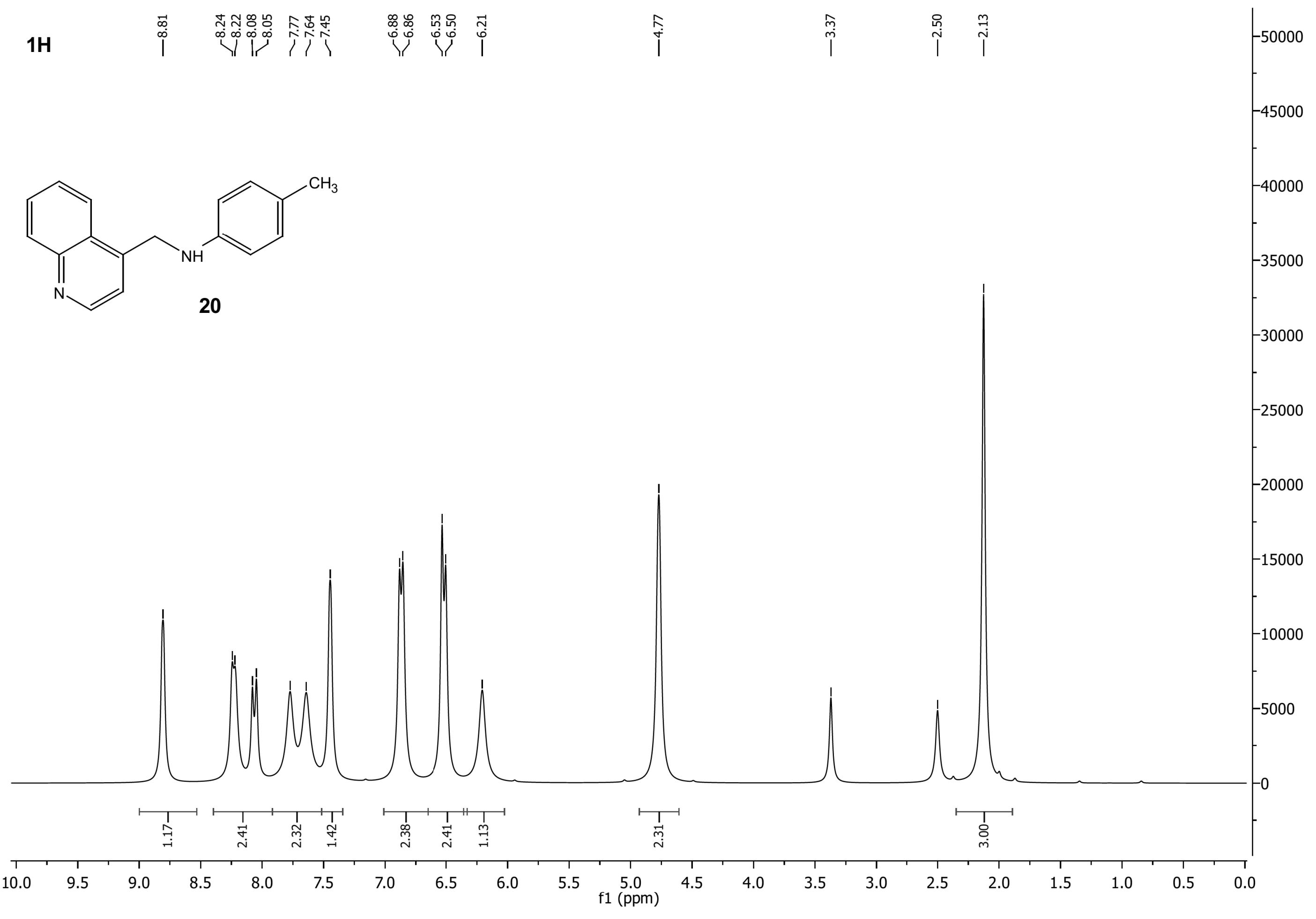




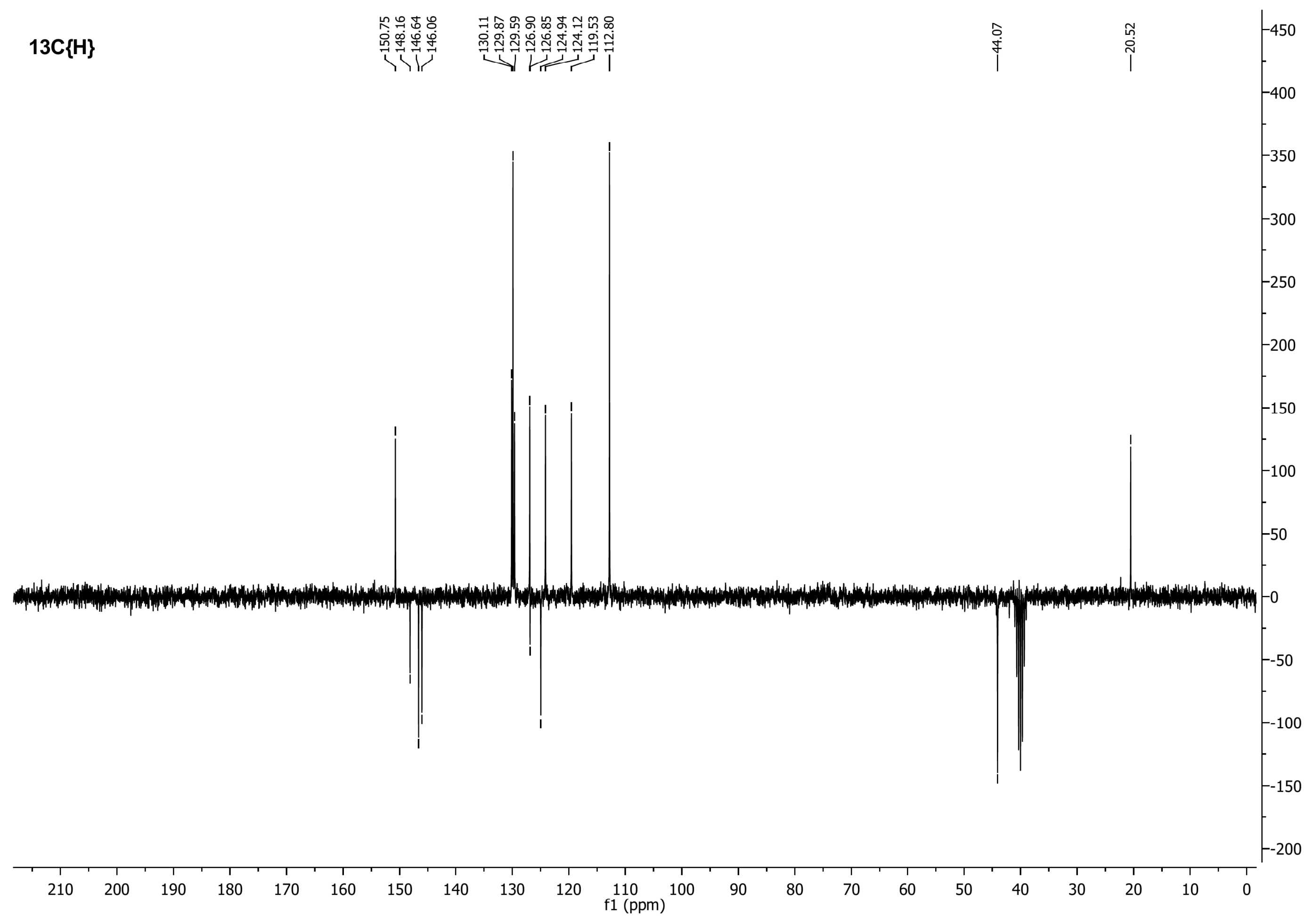




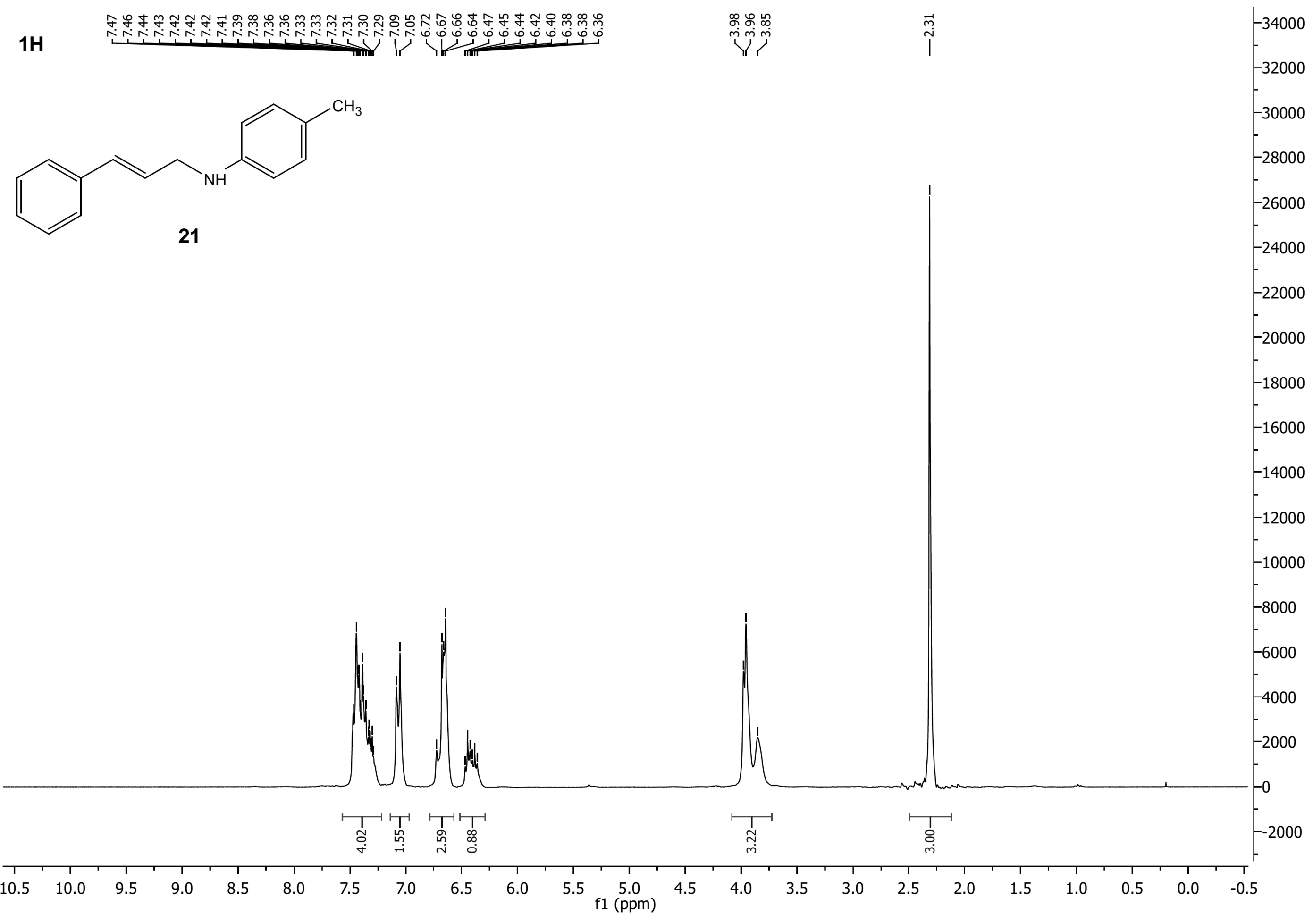




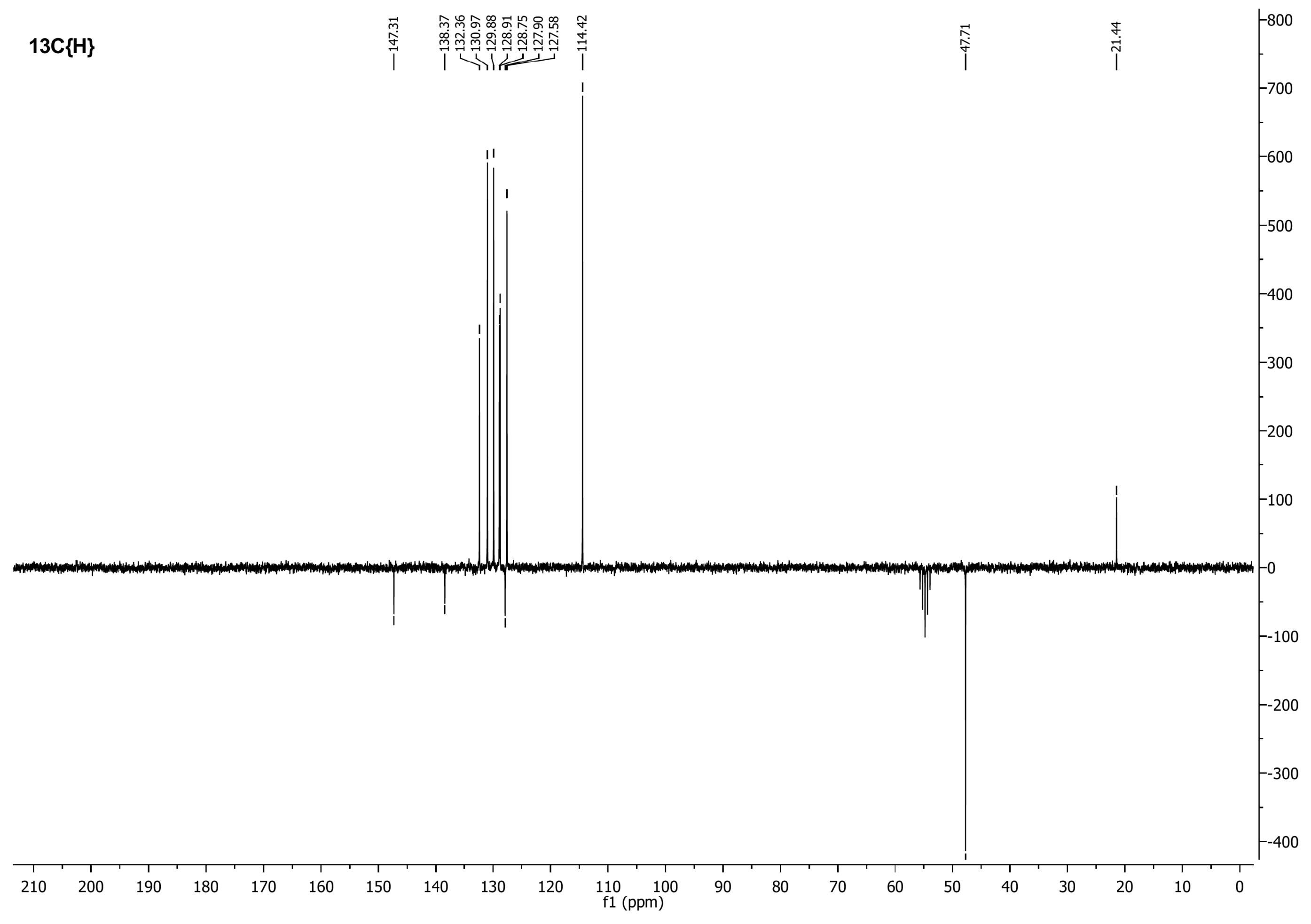




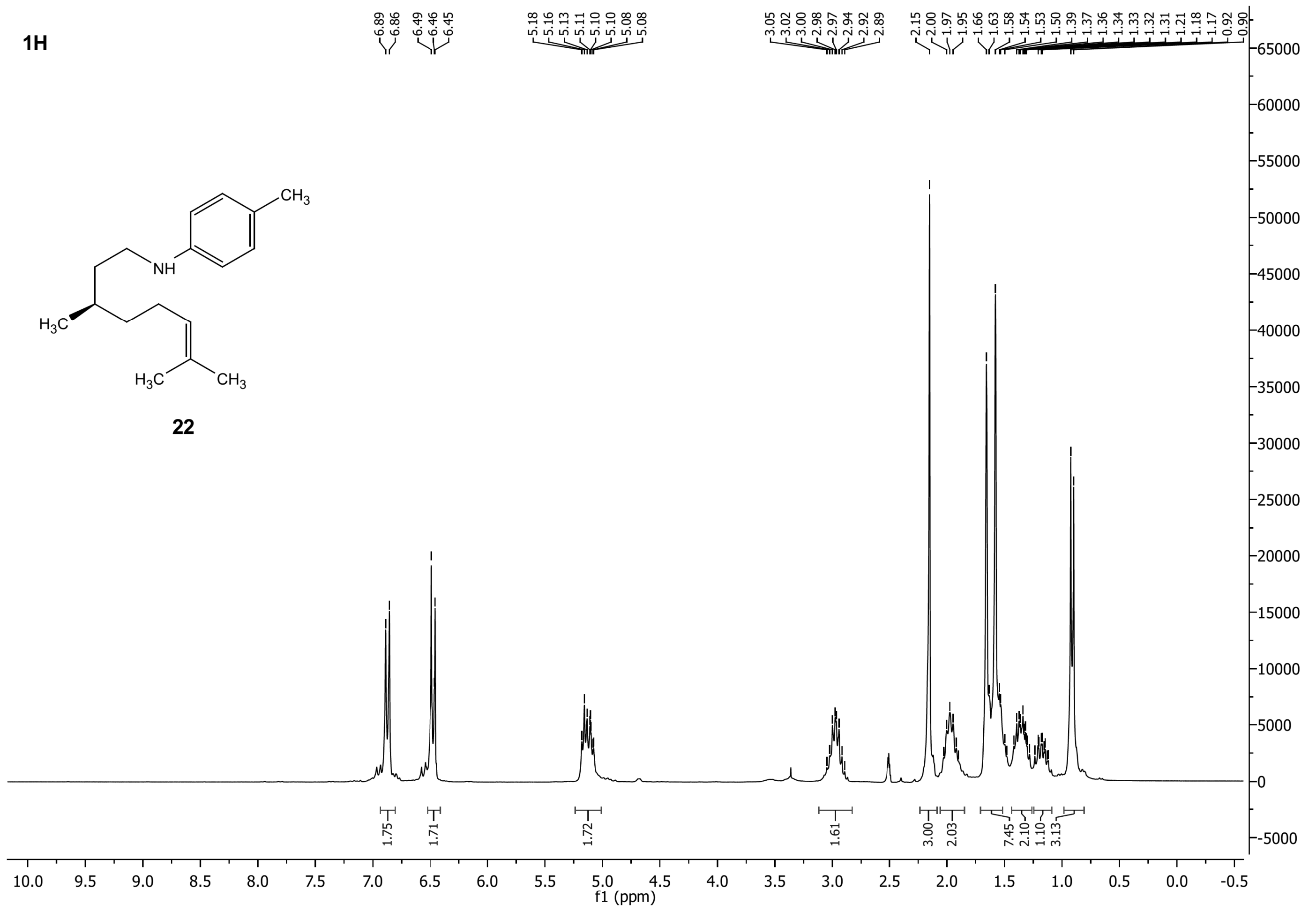




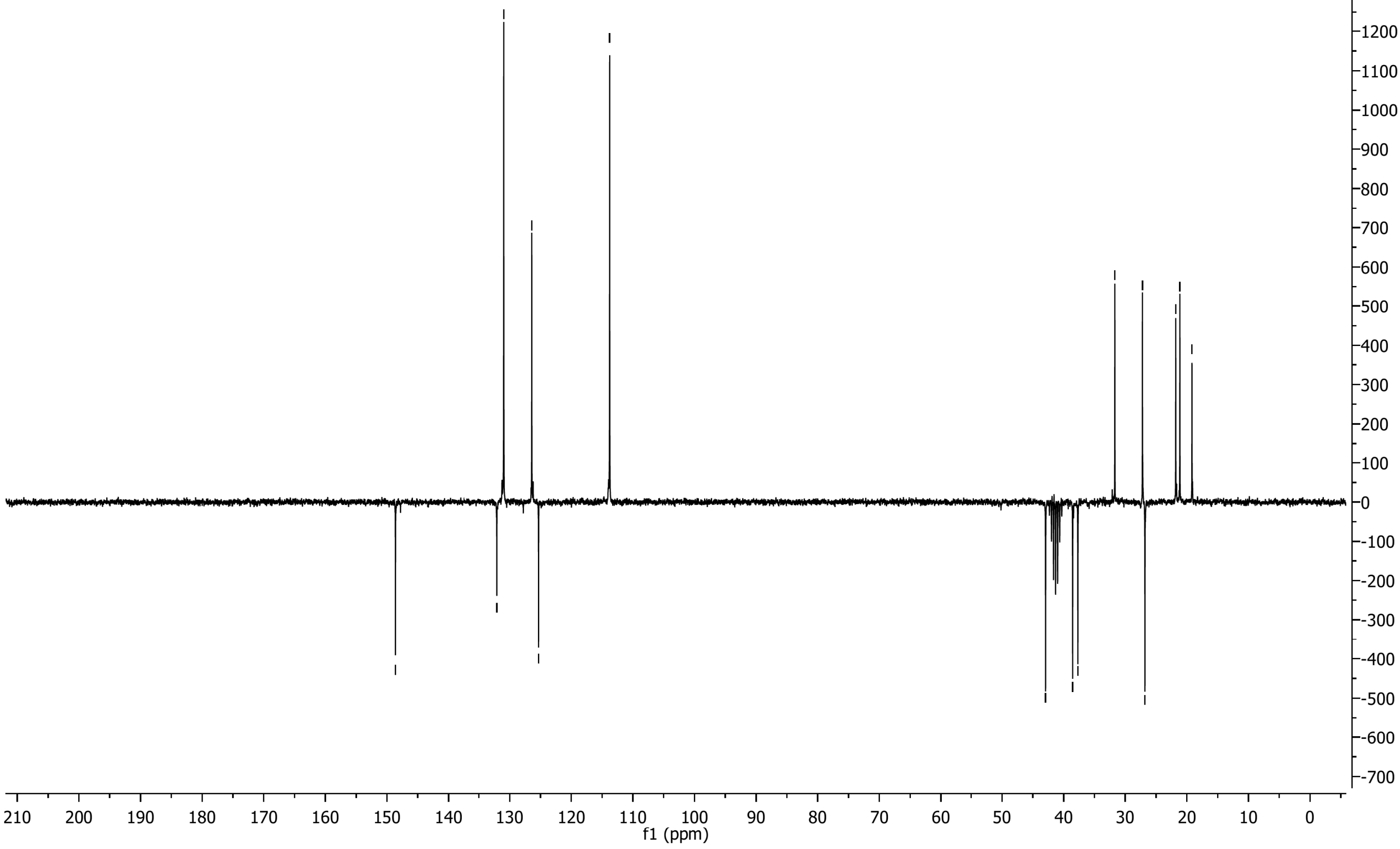




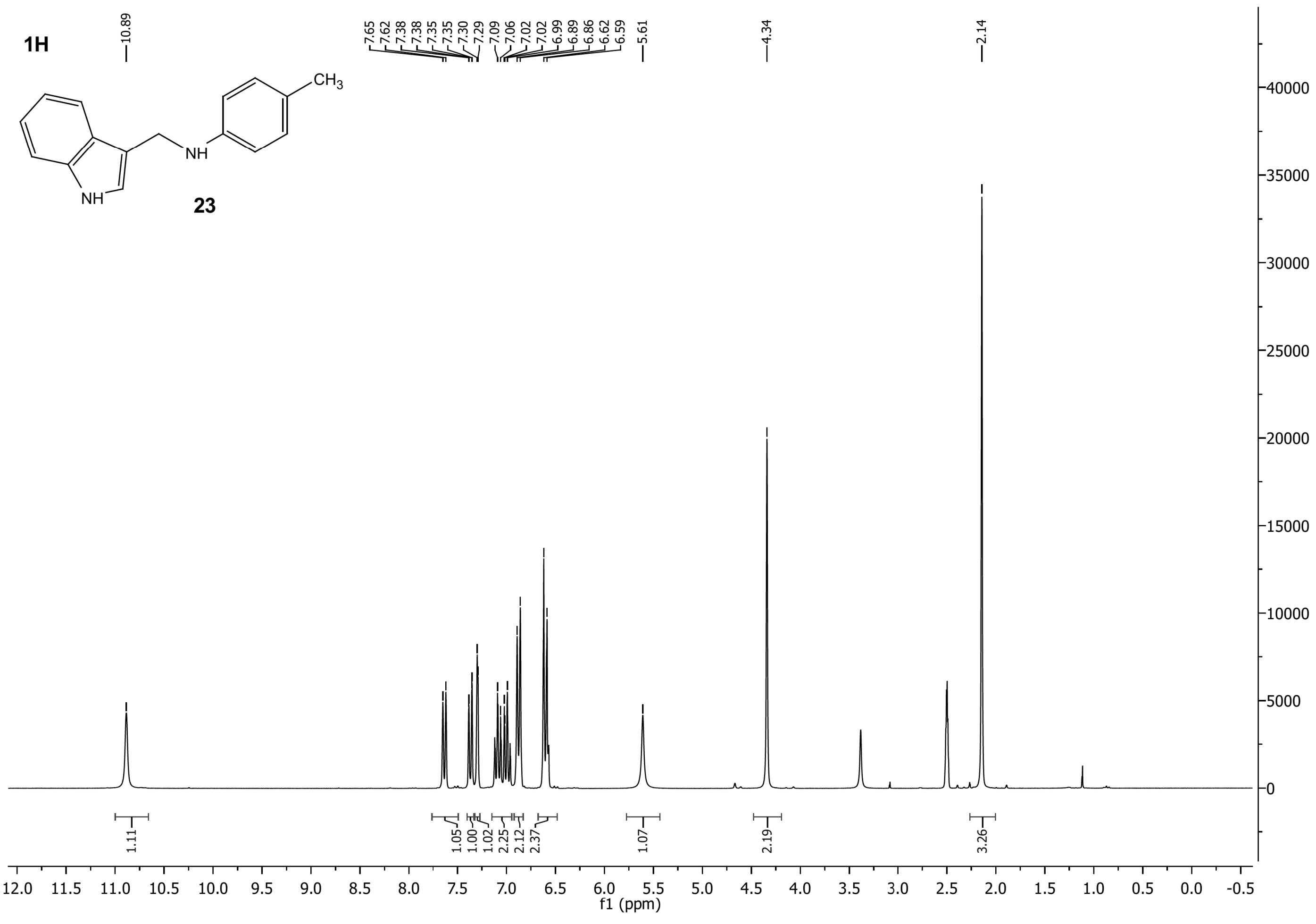




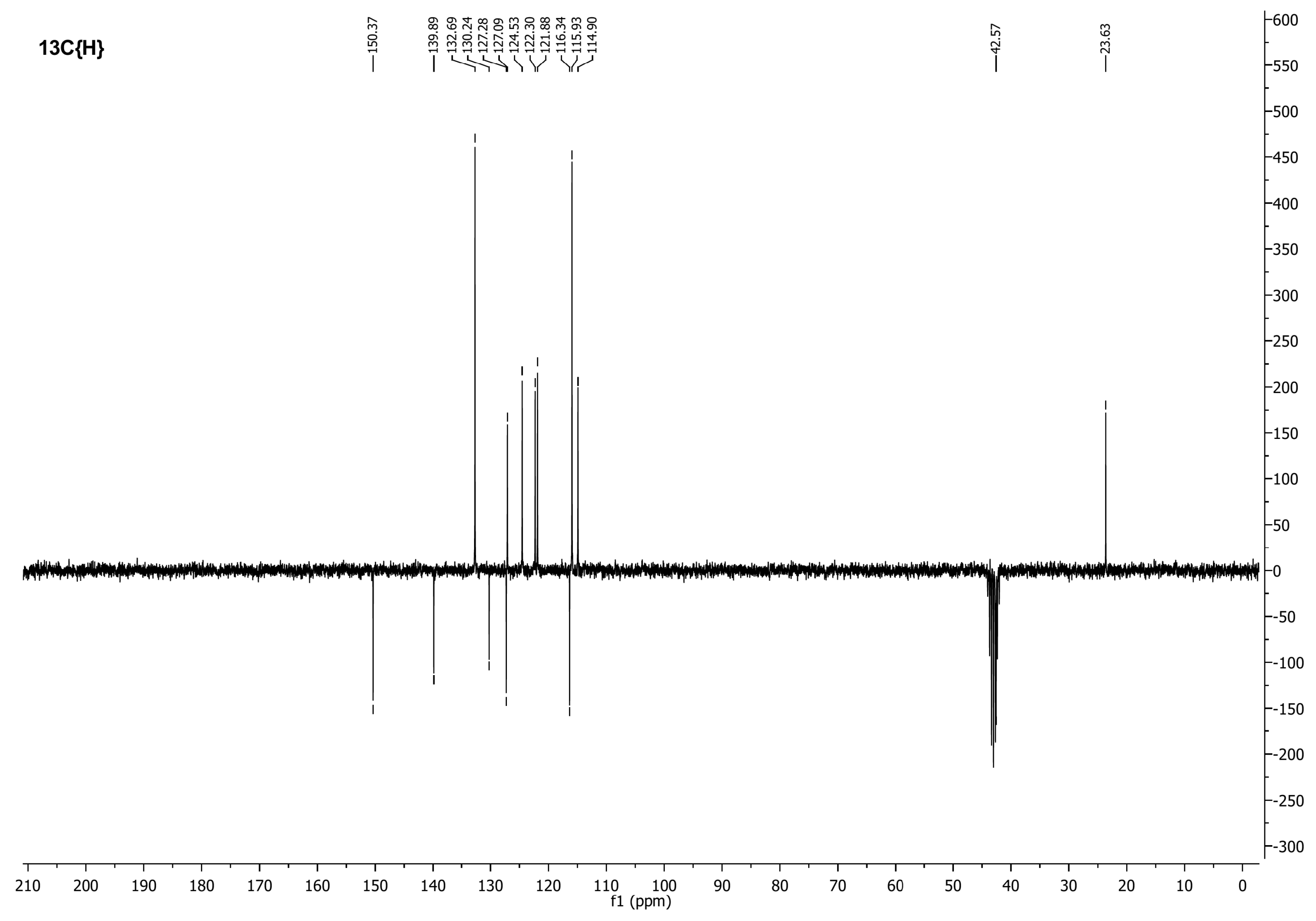




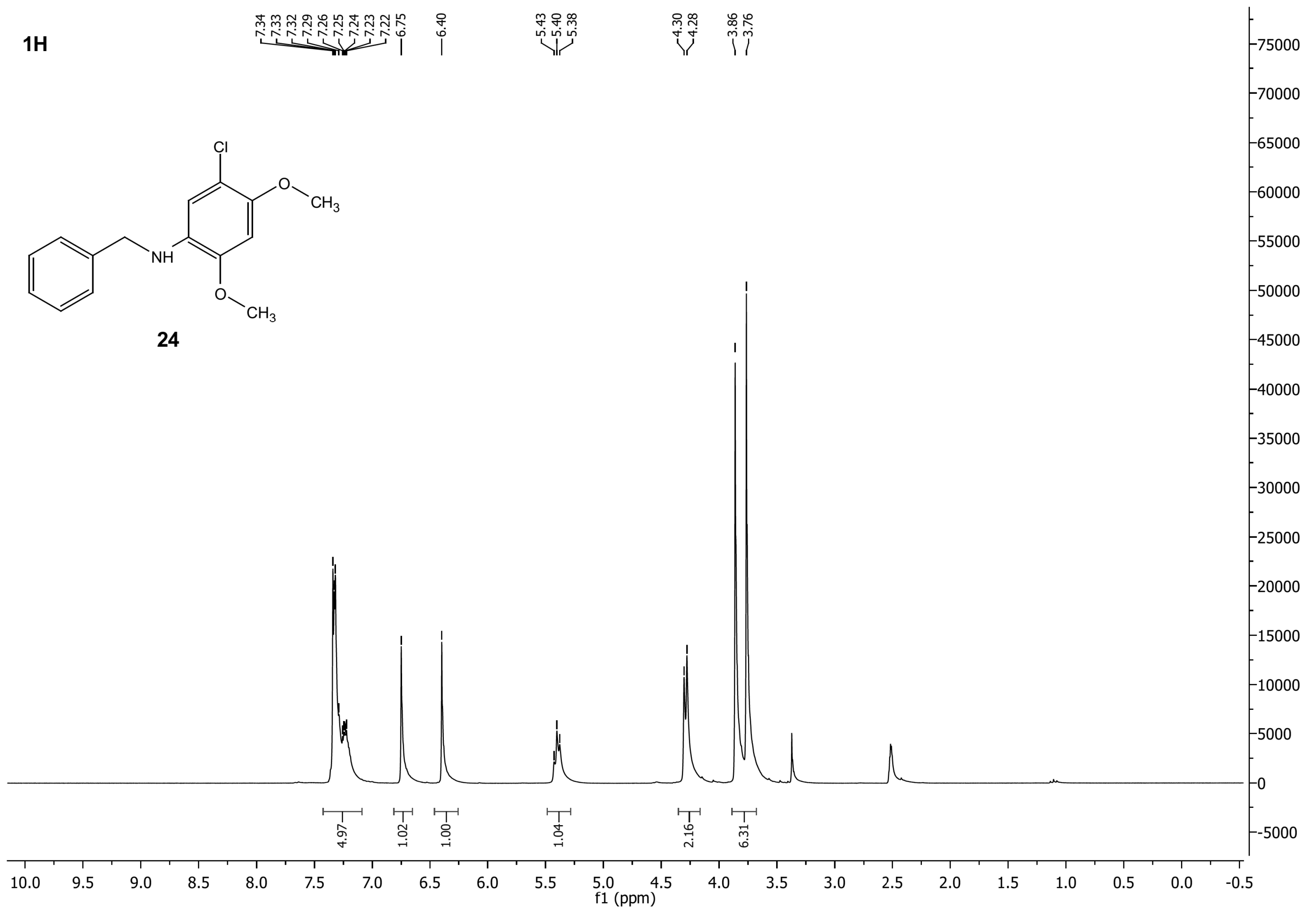




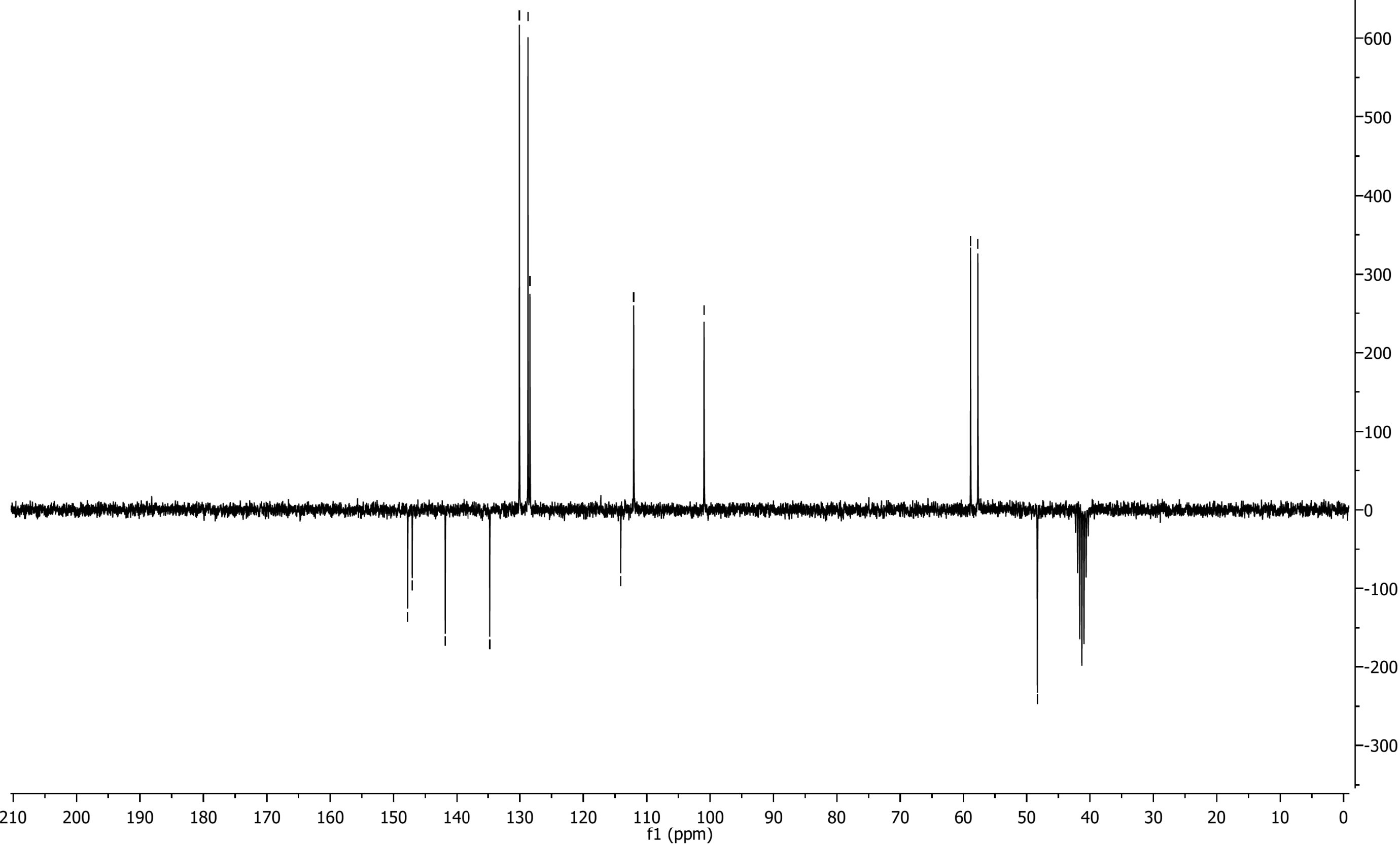




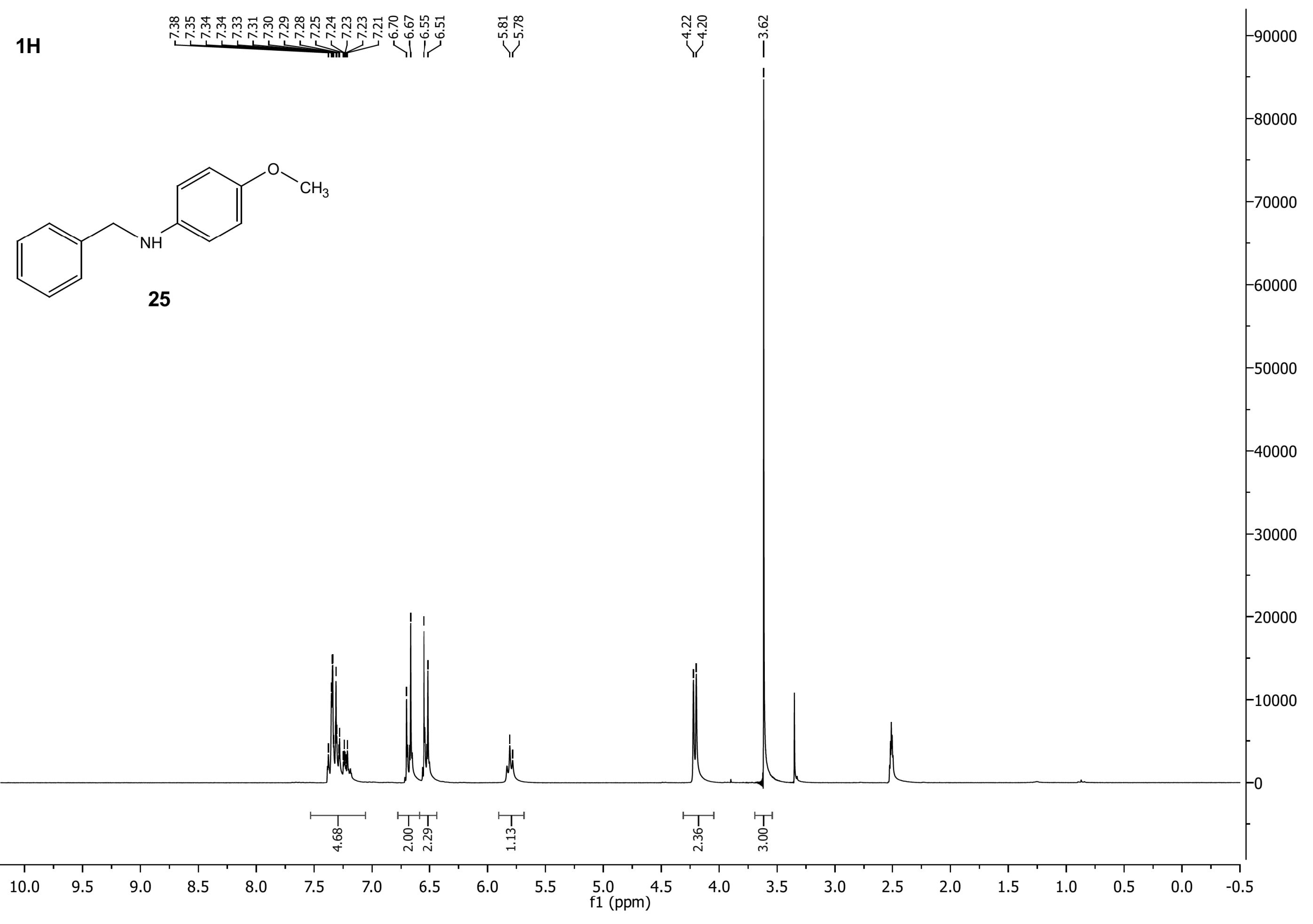




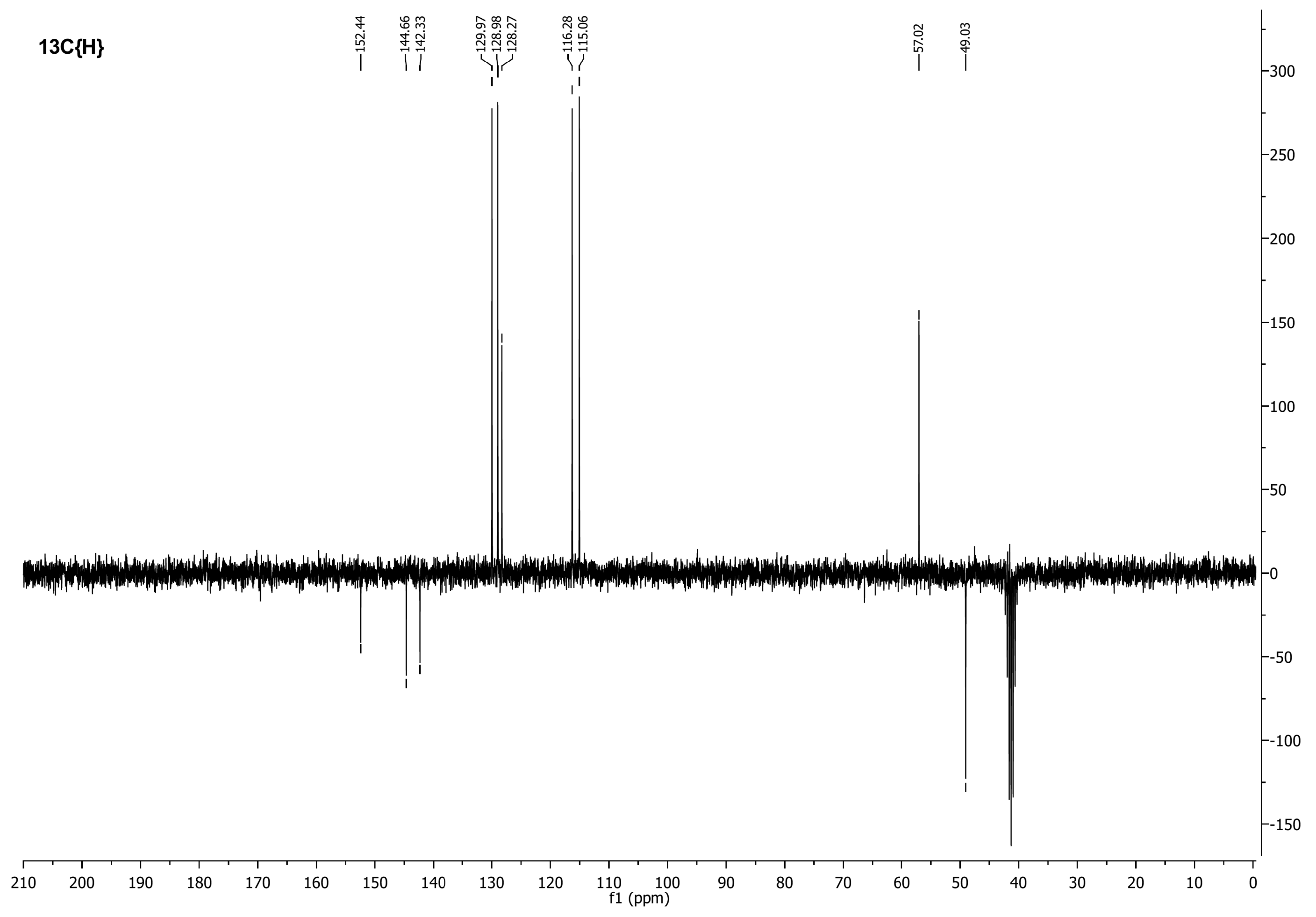




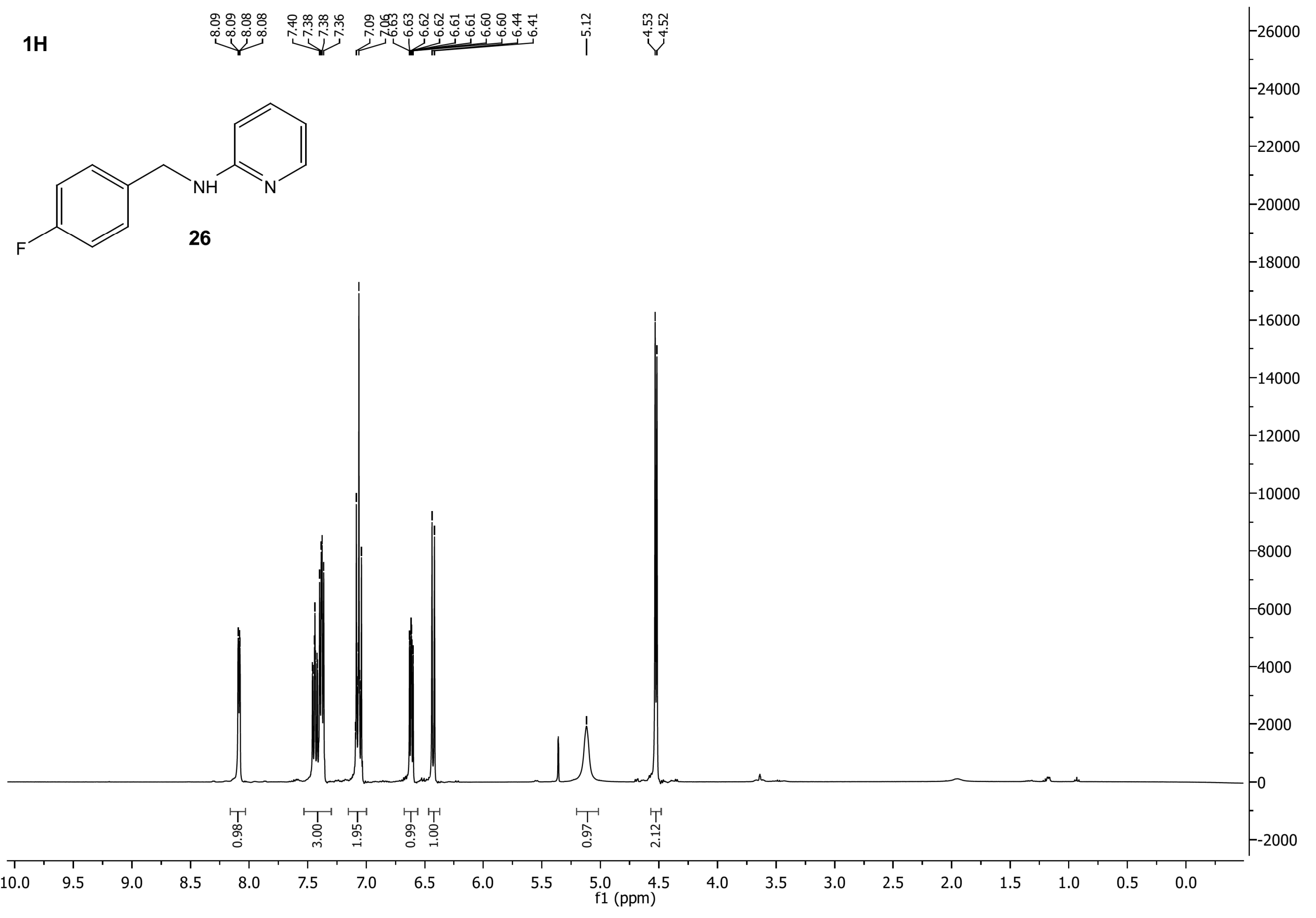




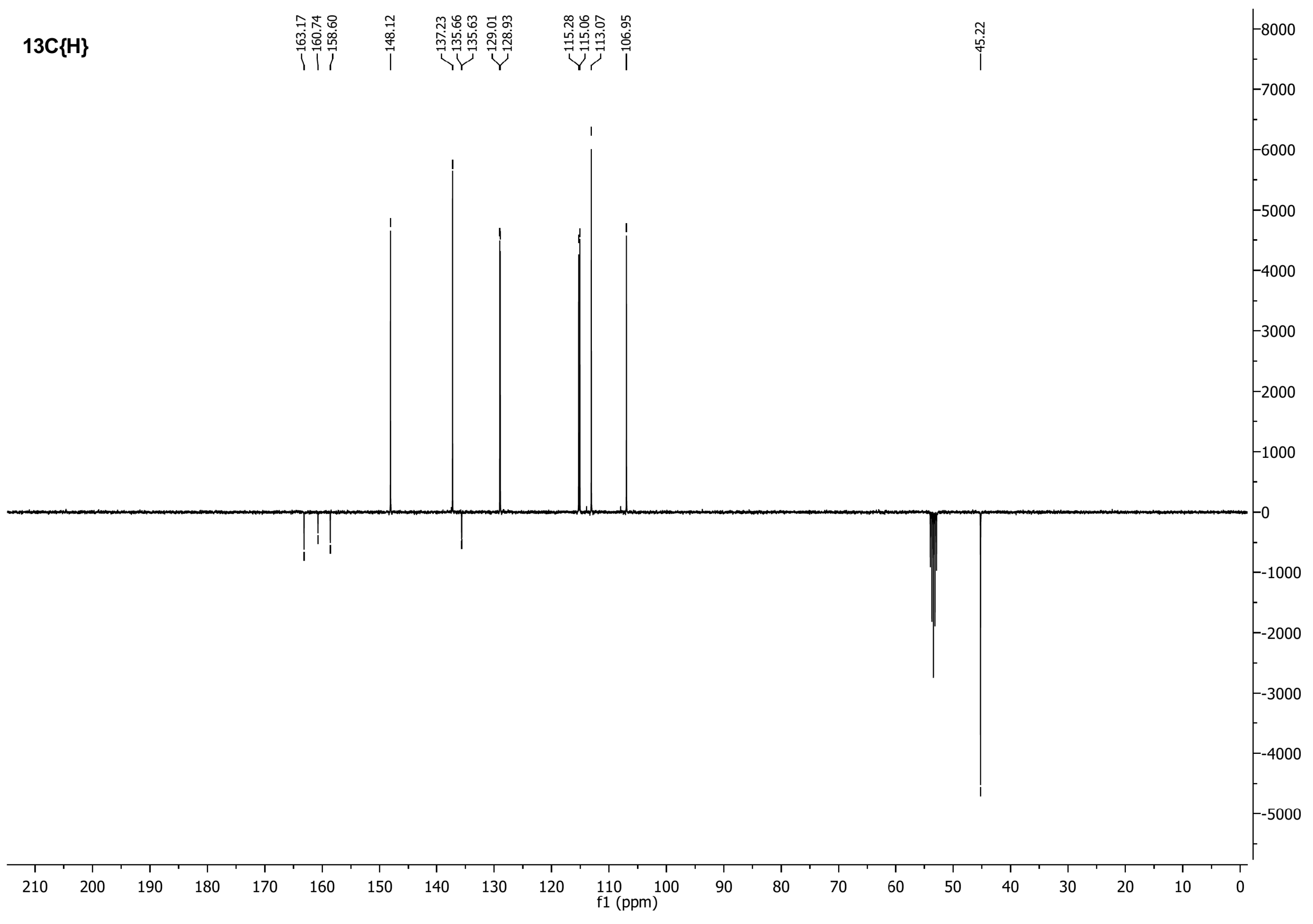




\section{$19 F\{\mathrm{H}\}$}

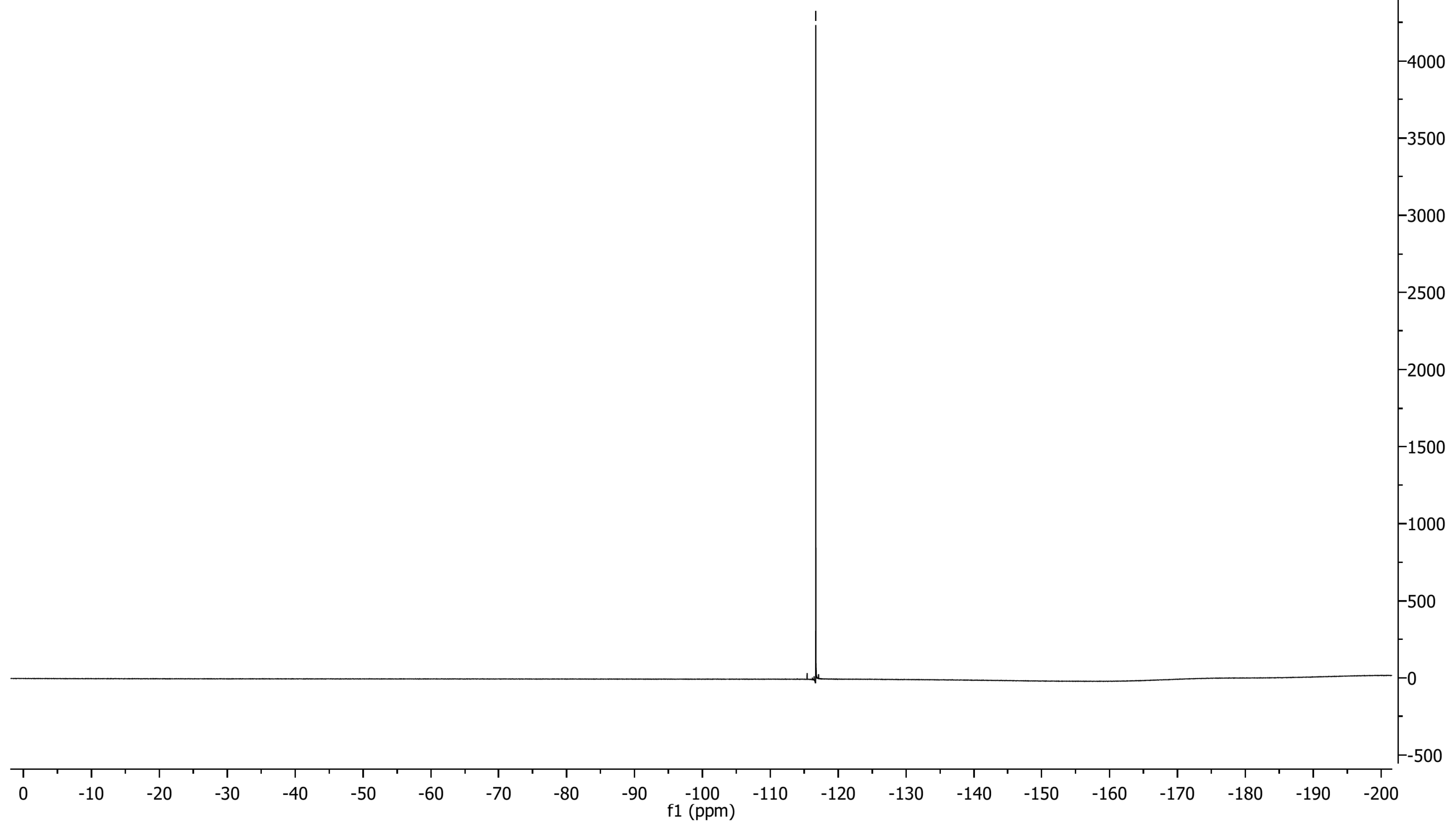




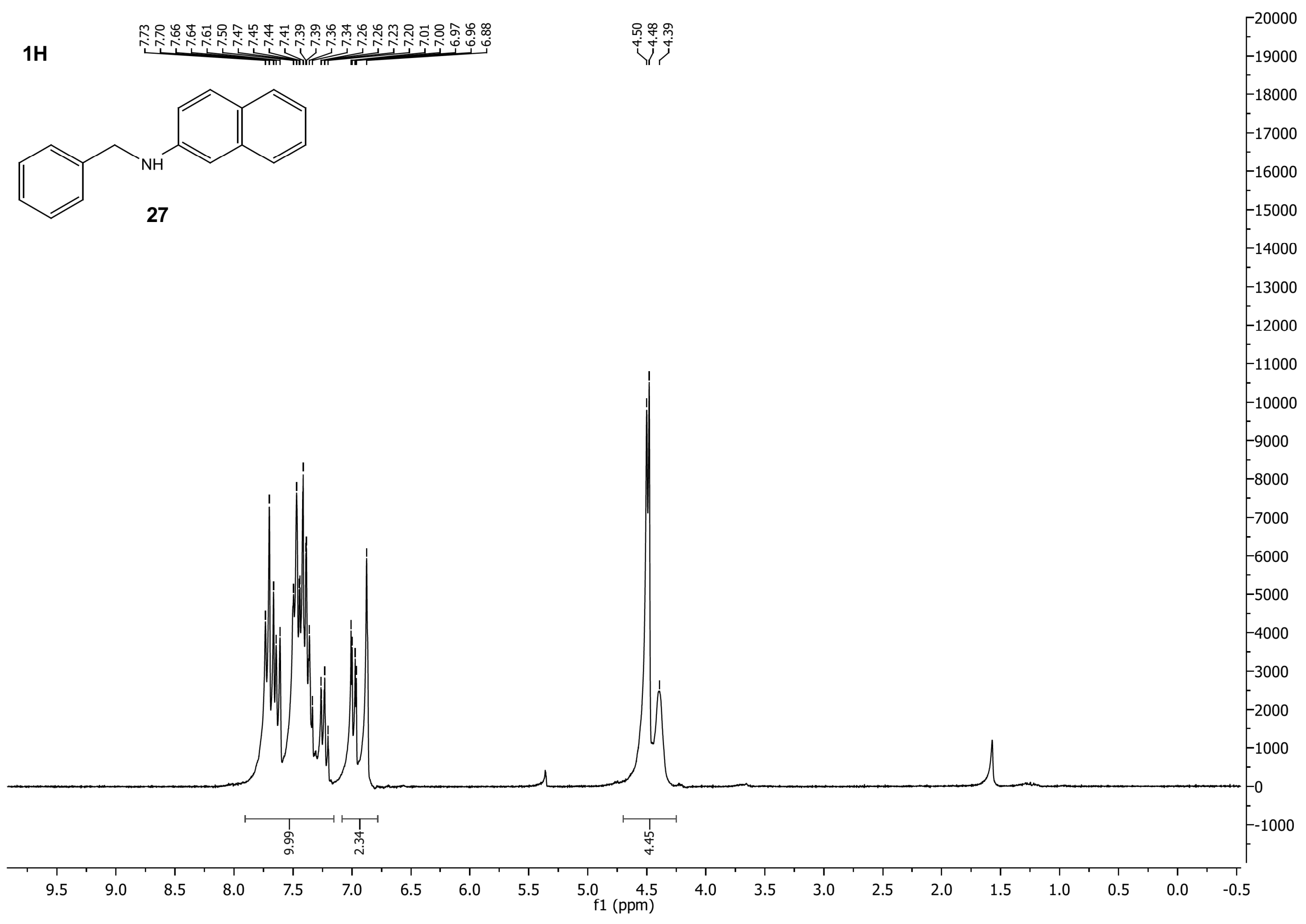




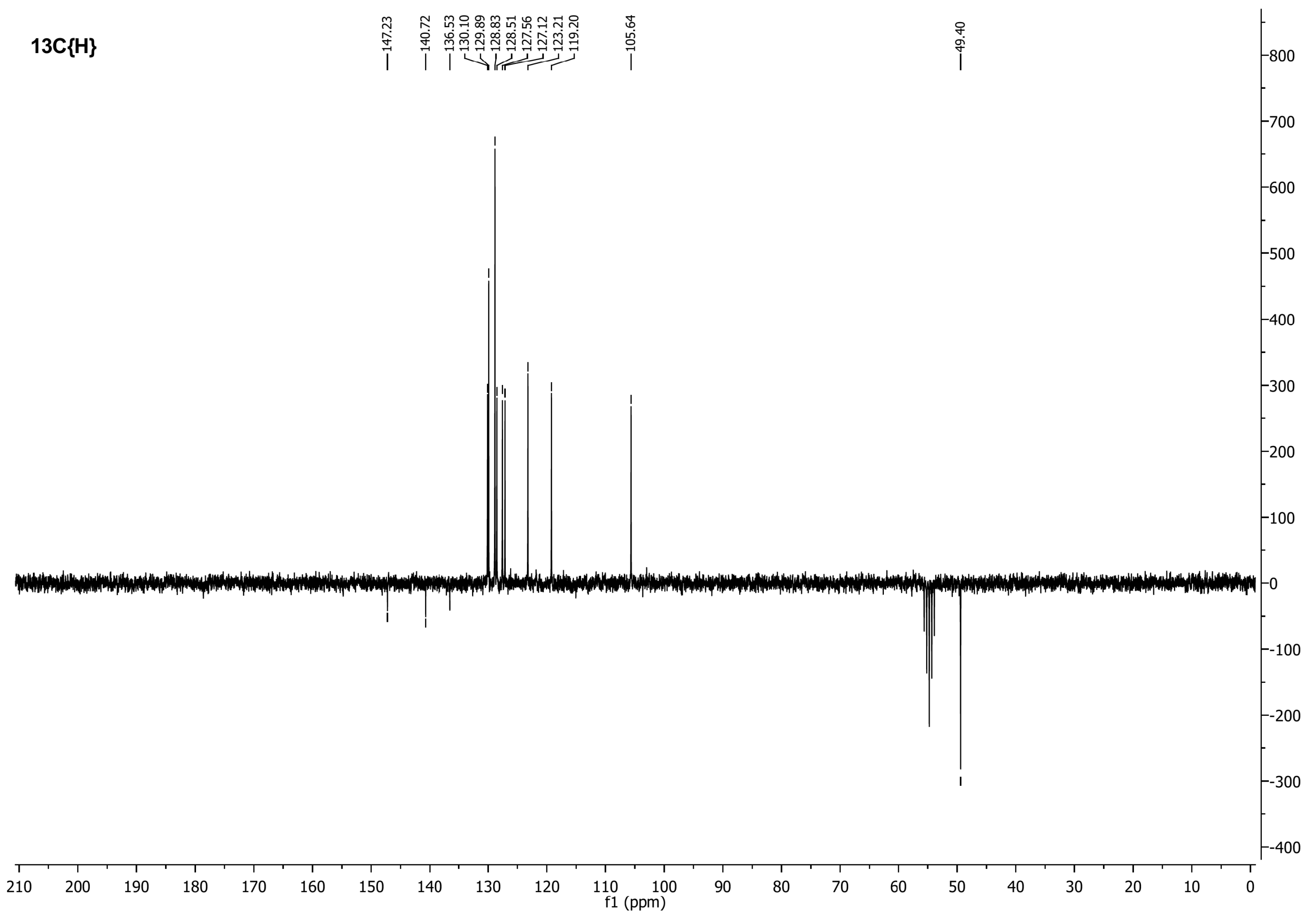




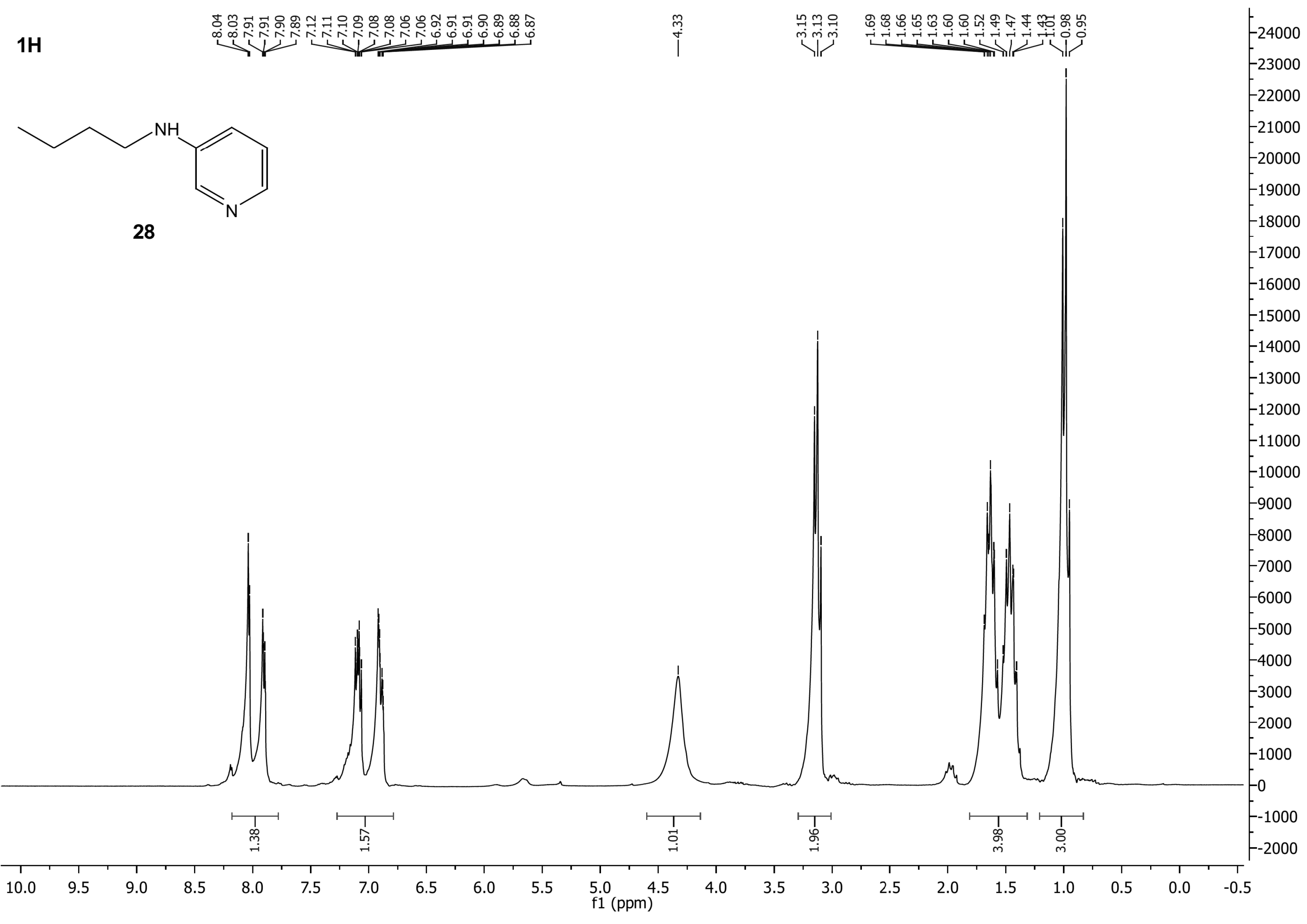




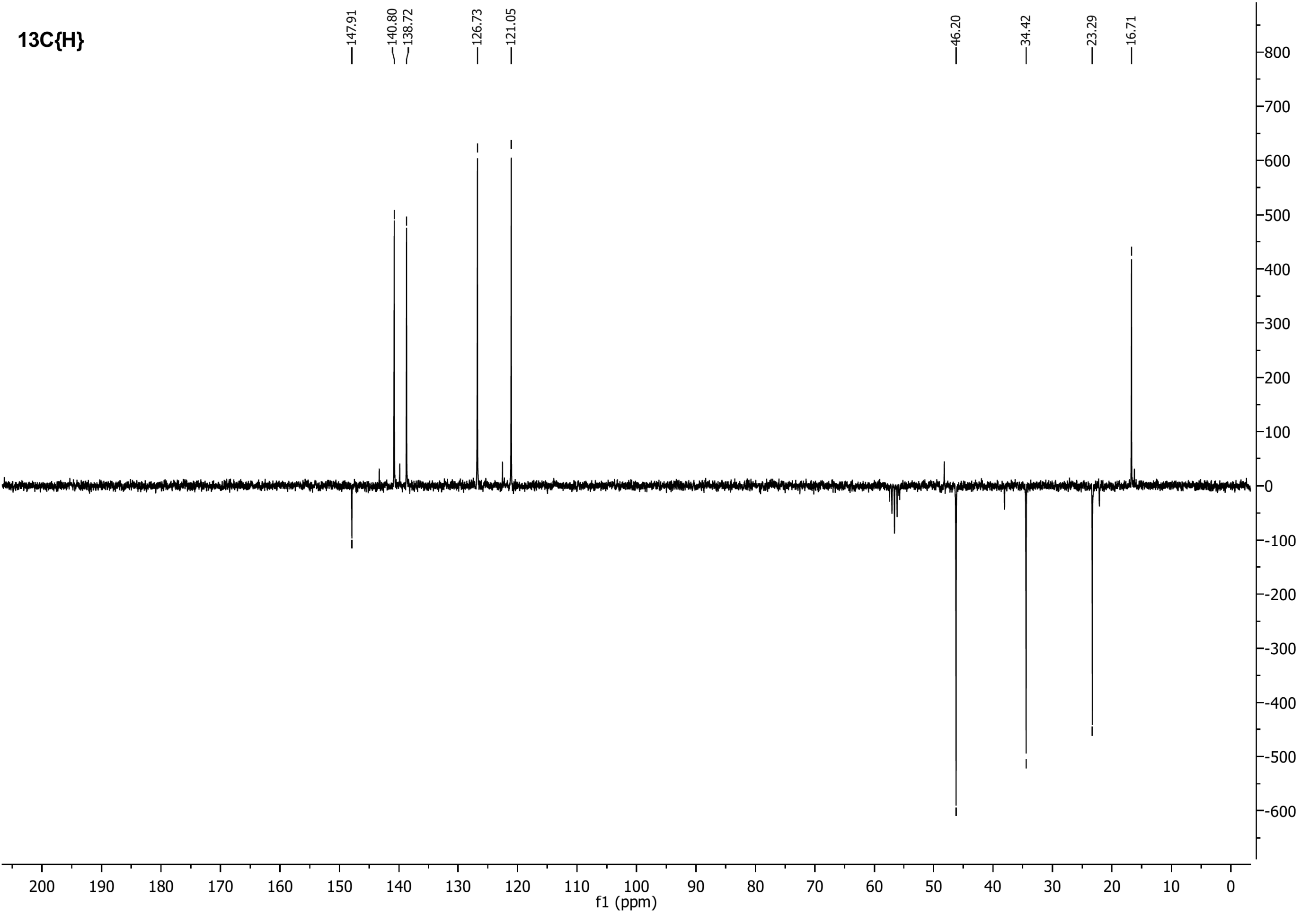




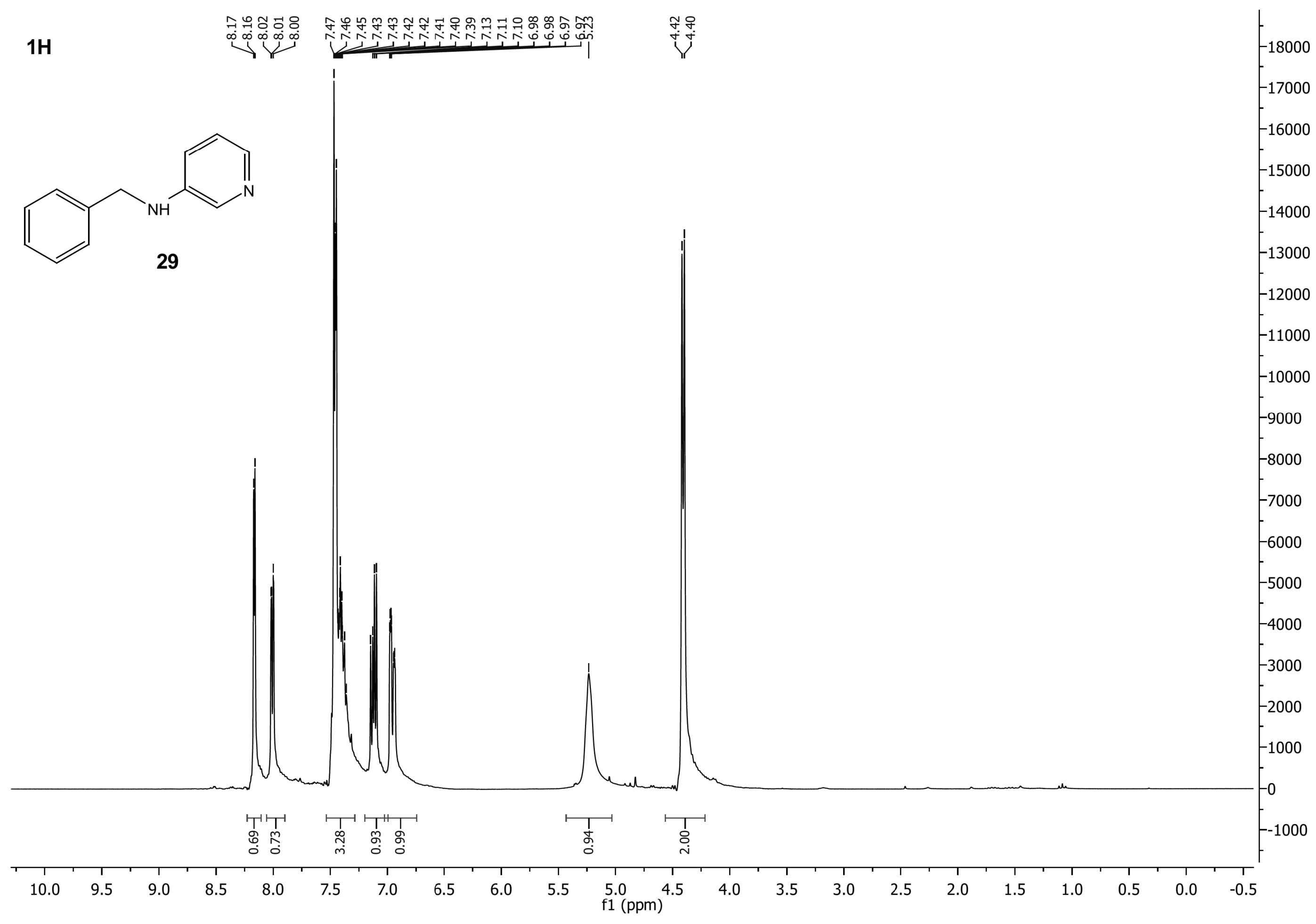




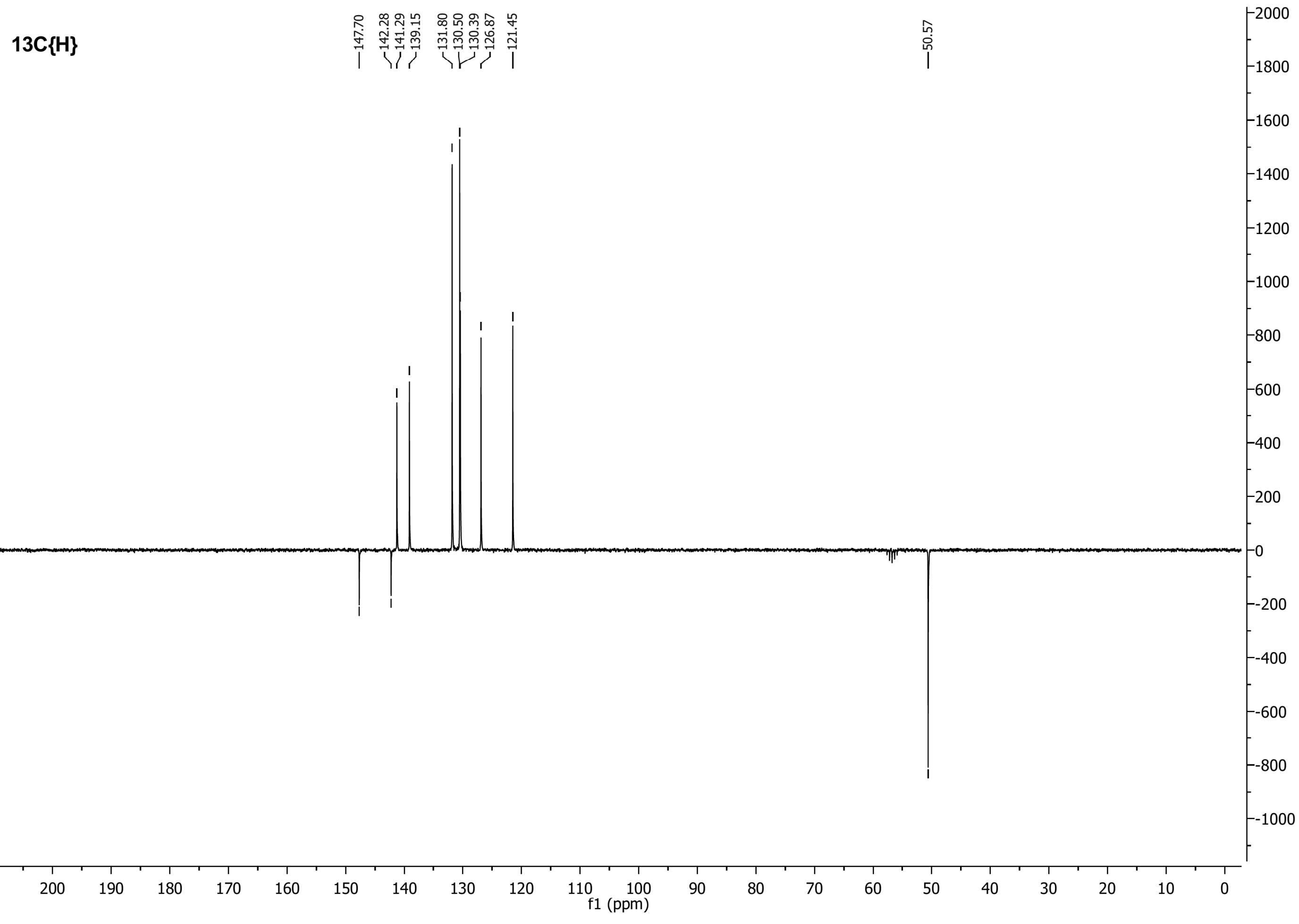




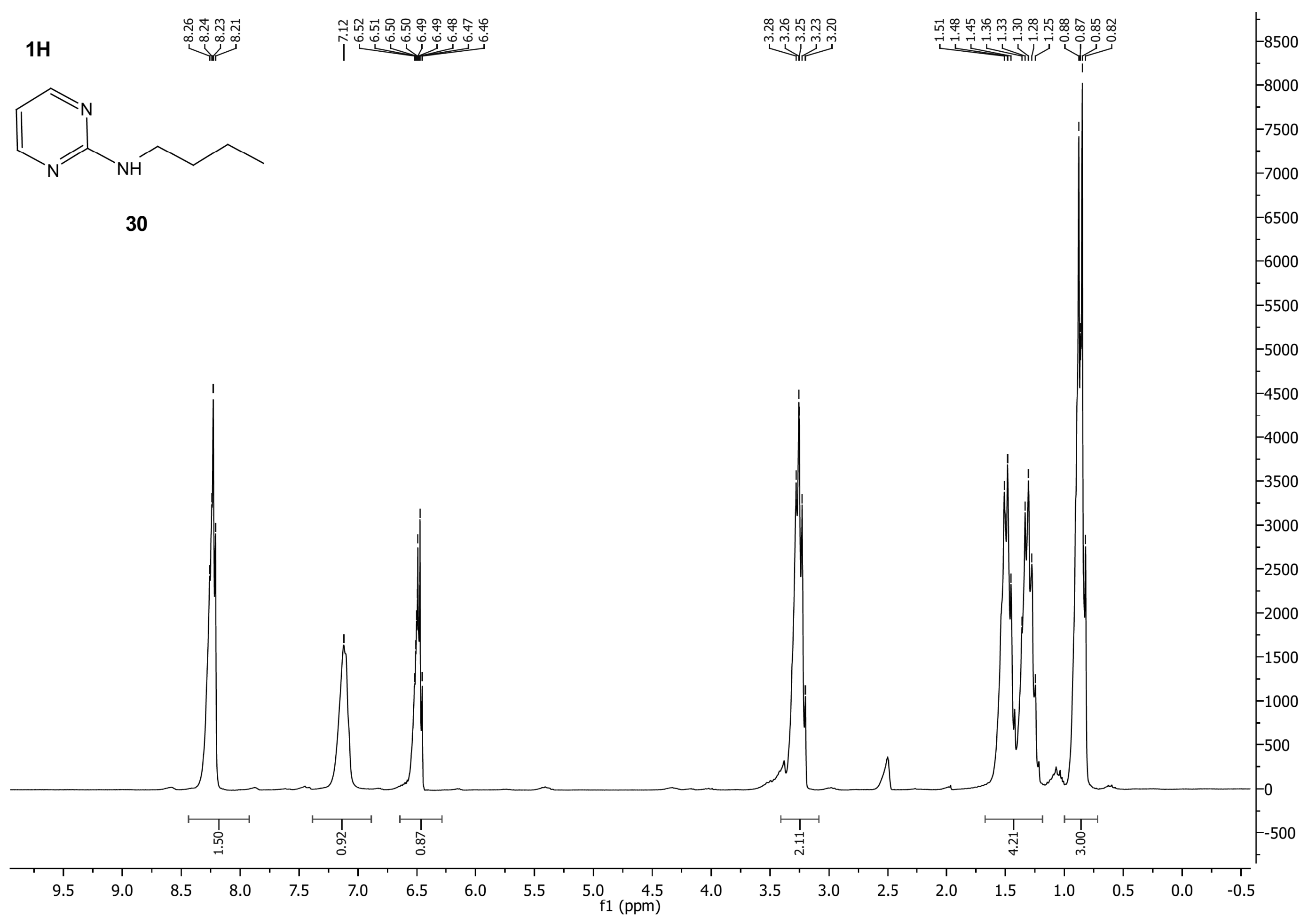




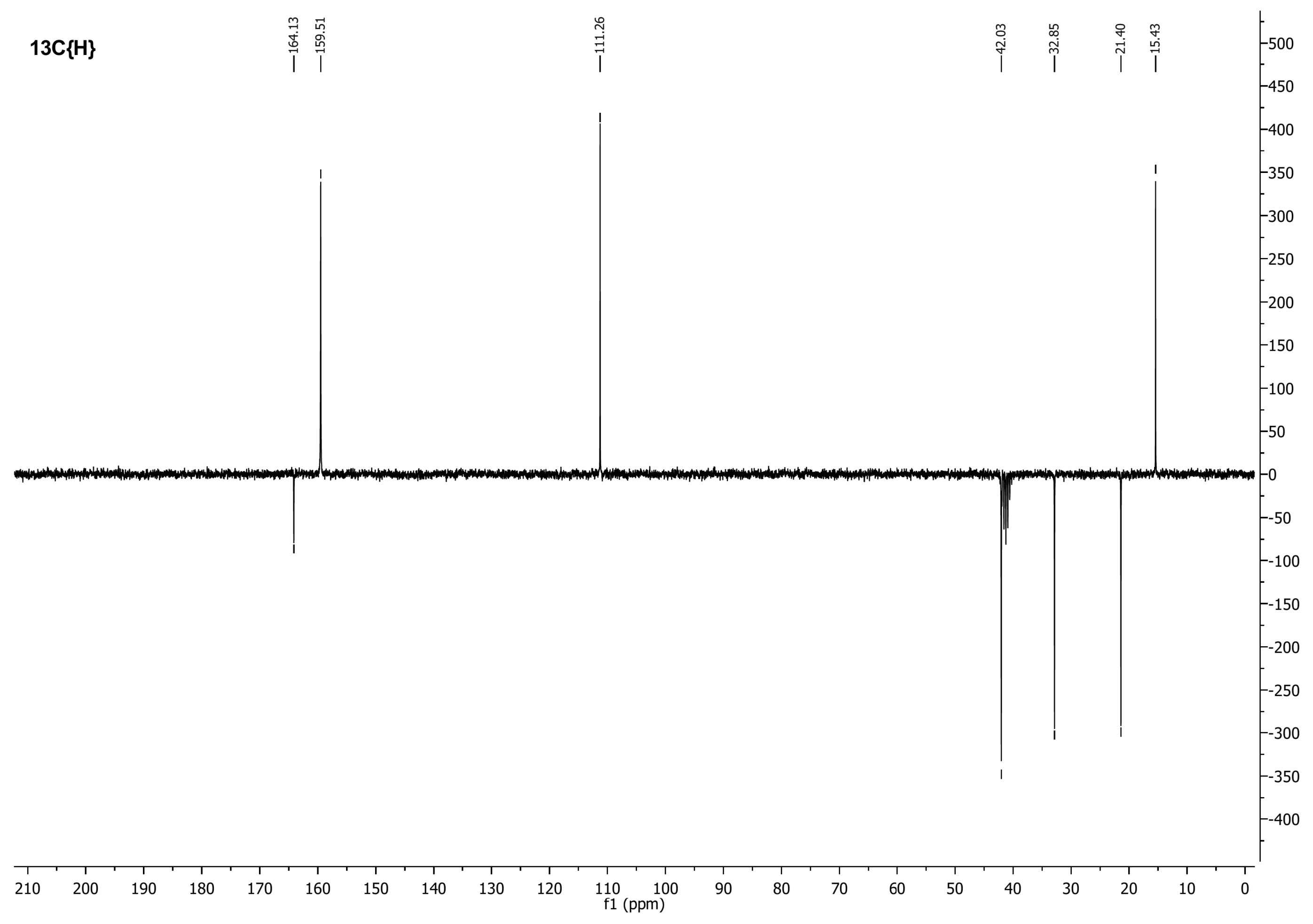




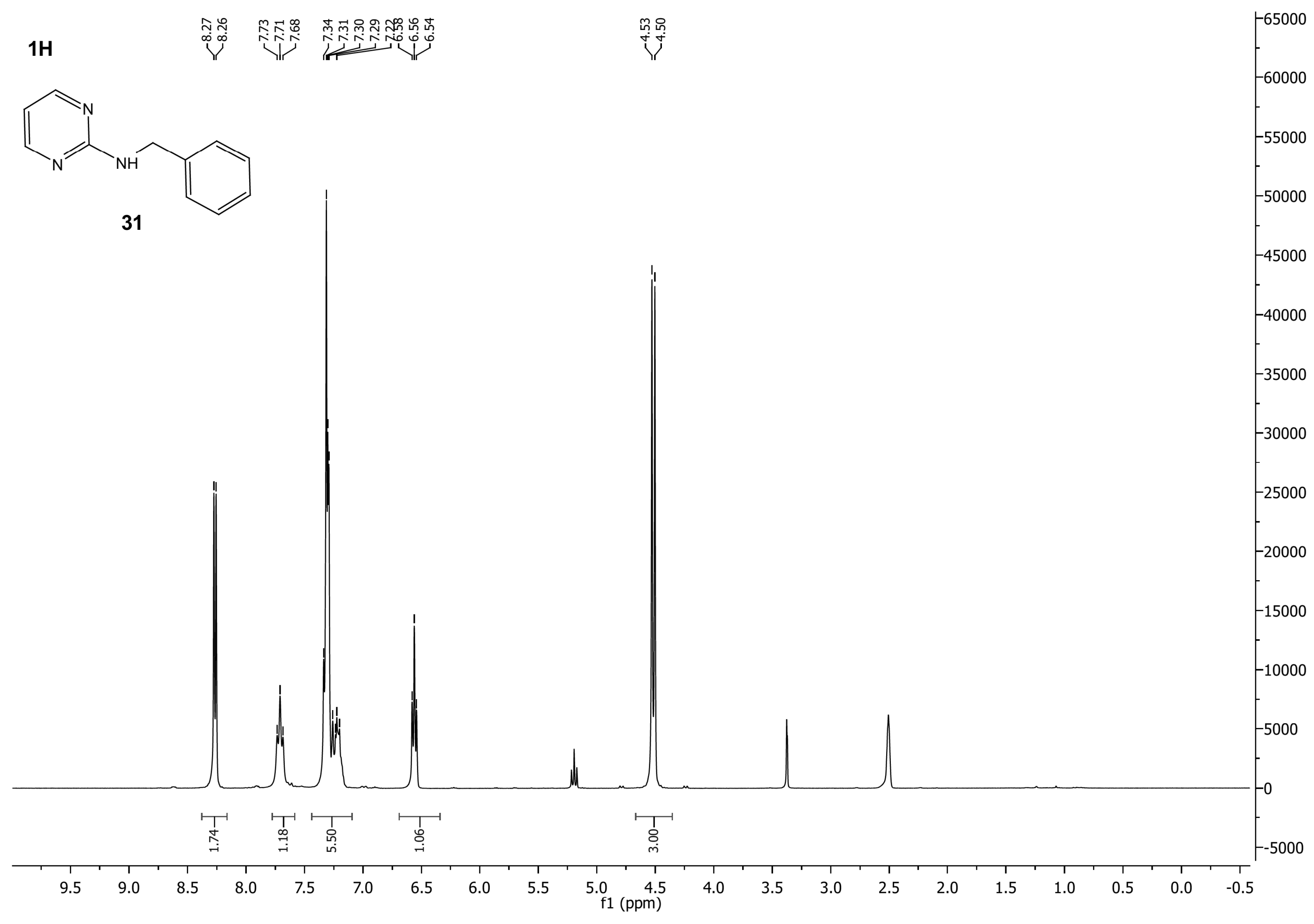




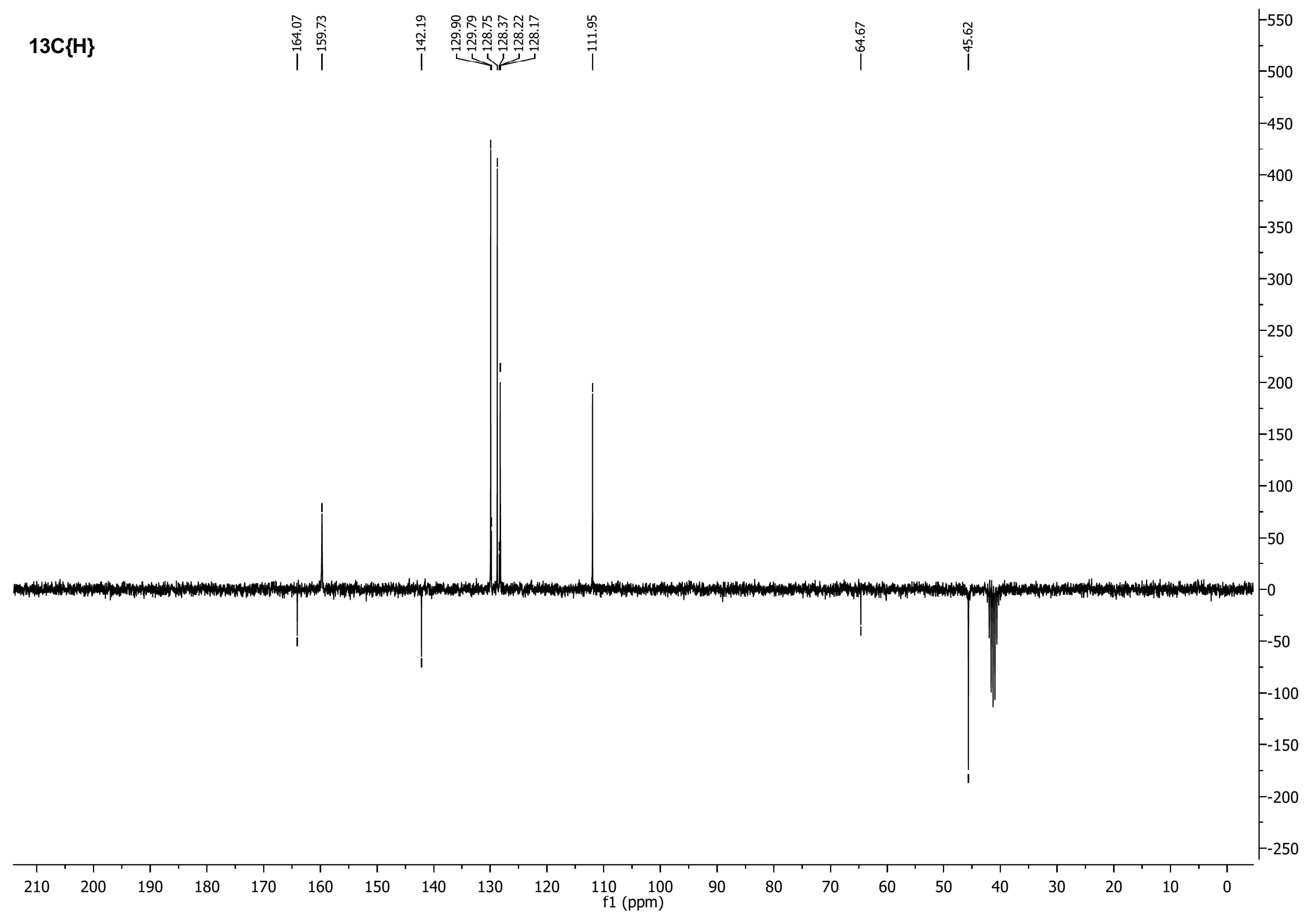




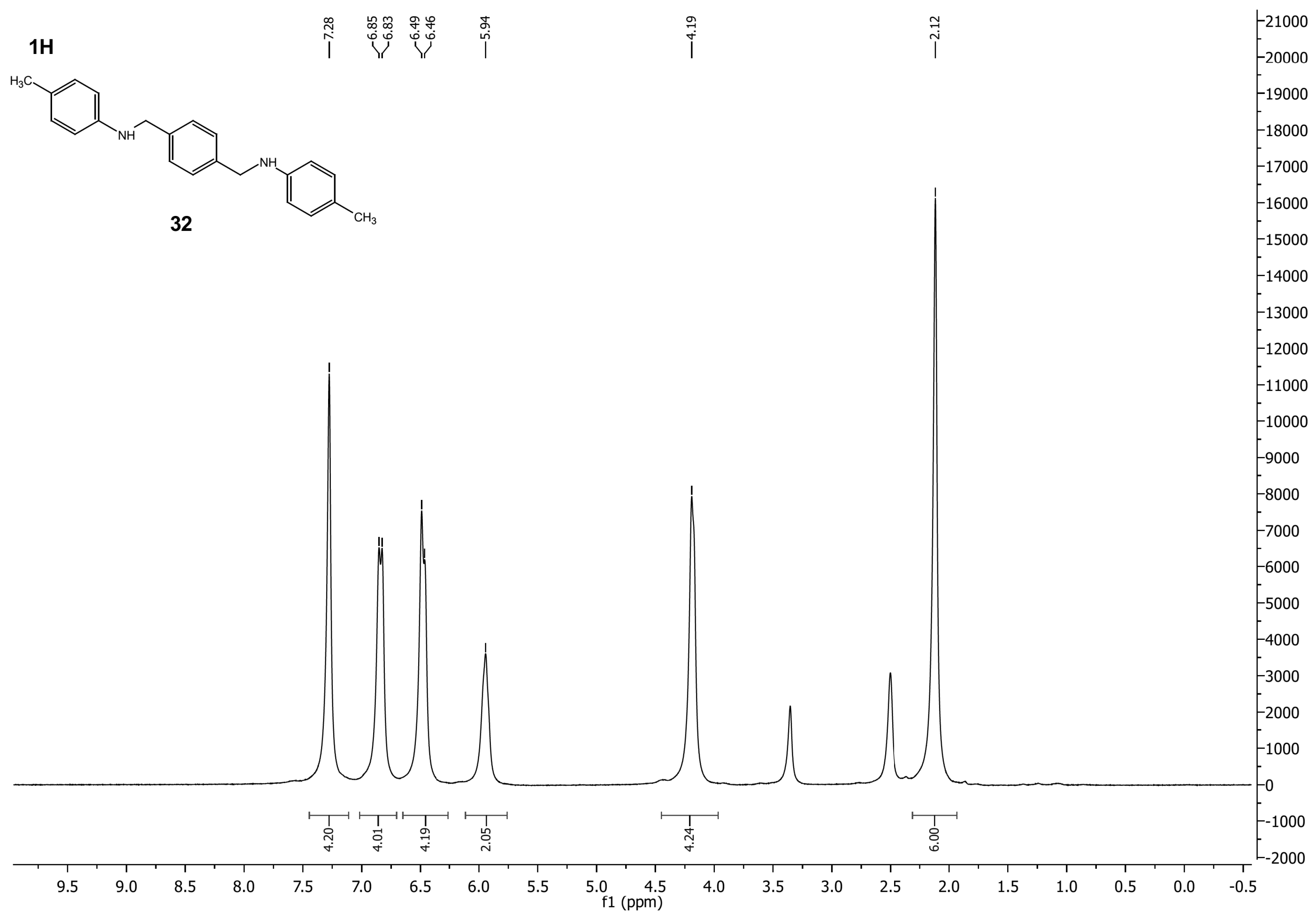




$$
\text { ll }
$$

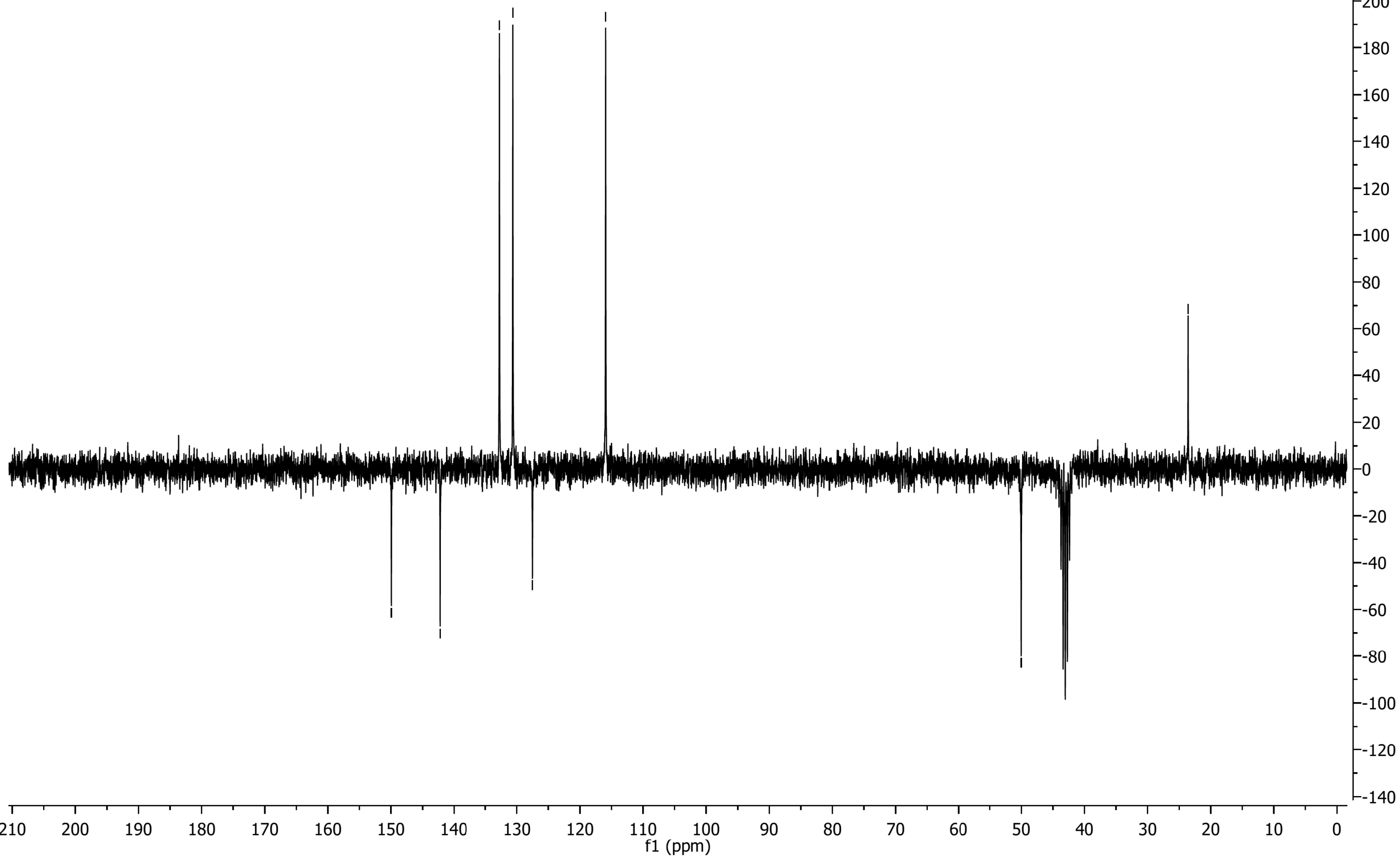

